\title{
Fördert die Nutzung von außerunterrichtlichen Bildungs- und Betreuungsangeboten an Tagesschulen soziale Kompetenzen?
}

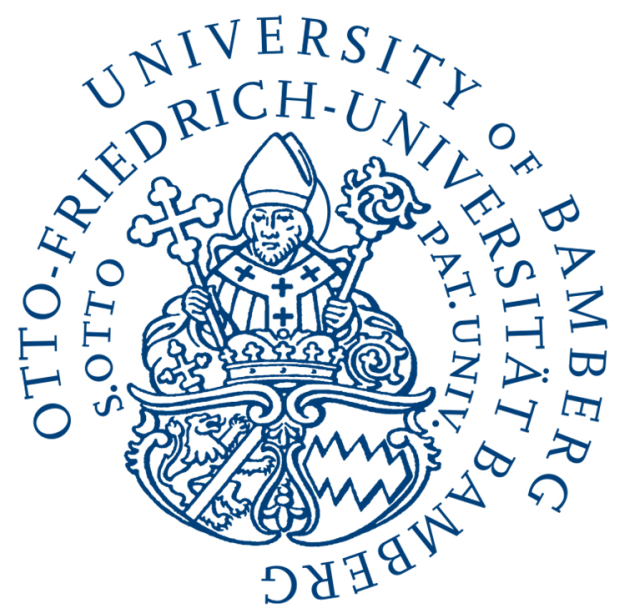

\section{Inaugural-Dissertation}

in der Fakultät Humanwissenschaften der Otto-Friedrich-Universität Bamberg

vorgelegt von

Lukas Frei

aus Adelboden

Bamberg, den 05.08.2021 
Dekan:

Erstgutachterin:

Zweitgutachterin:
Prof. Dr. Jörg Wolstein

Prof. Dr. Marianne Schüpbach

Prof. Dr. Barbara Drechsel

Dieses Werk ist als freie Onlineversion über das Forschungsinformationssystem (FIS; https://fis.uni-bamberg.de) der Universität Bamberg erreichbar. Das Werk steht unter der CC-Lizenz CC-BY-NC-ND.

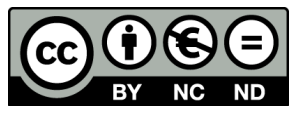

URN: urn:nbn:de:bvb:473-irb-509741

DOI: https://doi.org/10.20378/irb-50974 


\section{Danksagung}

An dieser Stelle möchte ich mich für all die Unterstützung bedanken, die ich beim Erarbeiten dieser Dissertation erfahren habe. Ein besonderes Dankeschön geht dabei an Marianne Schüpbach, für die tolle Betreuung meiner Arbeit. Ich konnte nicht nur auf wertvolle Rückmeldungen und Impulse zählen, sondern wurde auch immer wieder motiviert, die nächsten Schritte zu planen und anzupacken. Als Projektleiterin von EduCare-TaSe organisierte sie wiederholt statistische und methodische Weiterbildungen, die gerade im Hinblick auf die vorliegenden Analysen eine riesige Bereicherung darstellten. Zudem konnte ich von zahlreichen Gelegenheiten profitieren, an Kongressen, Tagungen und Kolloquien teilzunehmen und meine Fortschritte im Arbeitsprozess zu präsentieren und zu diskutieren.

Auch bei meinem Arbeitskollegen, Benjamin von Allmen, bedanke ich mich herzlich für die gelungene Zusammenarbeit und all die formellen sowie informellen Anregungen und Rückmeldungen. Egal ob im Büro, in der Sporthalle, an Kongressen oder an Weiterbildungen habe ich unsere intensiven Diskussionen stets geschätzt.

Weiter möchte ich mich bei Wim Nieuwenboom für die statistische und methodische Unterstützung bedanken, bei meiner Zweitbetreuerin Barbara Drechsel für die konstruktiven Rückmeldungen während den Kolloquien, bei Nathalie Junker für das wiederholte Korrekturlesen und bei Rachael Kullmann für die Unterstützung bei den englischen Texten. 


\section{Inhalt}

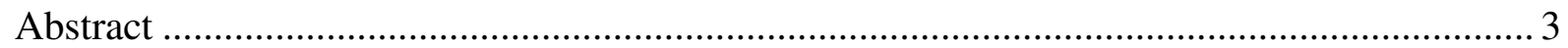

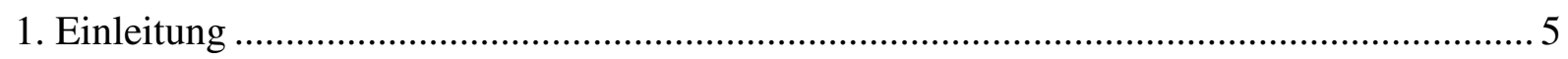

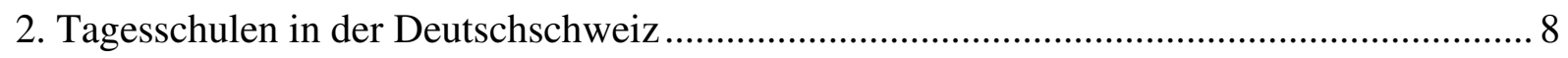

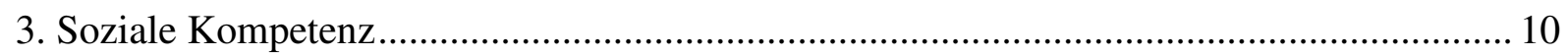

4. Fördert die Teilnahme am Tagesschulangebot soziale Kompetenzen?................................ 12

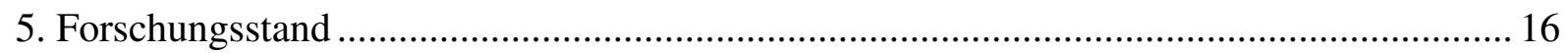

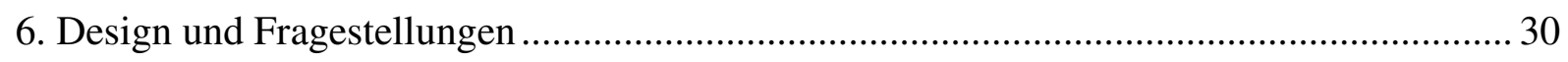

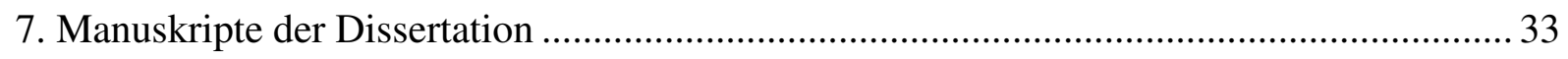

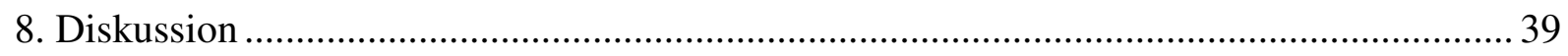



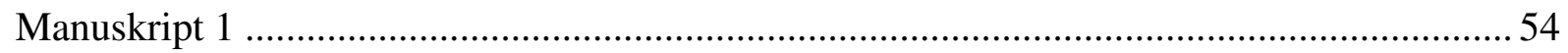

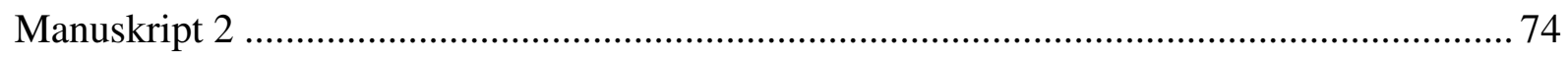

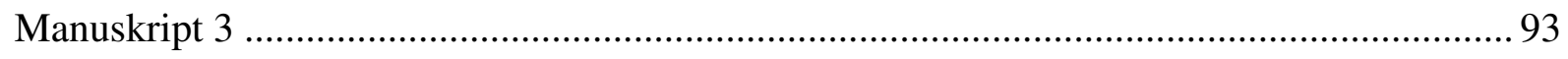

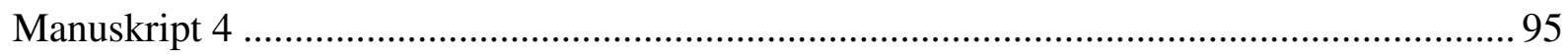

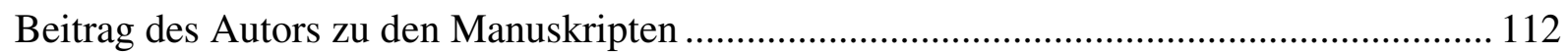




\begin{abstract}
Mit dem zunehmenden Ausbau von Tagesschulen in der Deutschschweiz und von Ganztagsschulen in Deutschland stiegen auch die Erwartungen an eine stärkere Förderung von sozialen Kompetenzen bei den Schülerinnen und Schülern. So sollen diese außerunterrichtlichen Bildungs- und Betreuungsangebote dazu beitragen, internalisierendes Verhalten (z.B. emotionale Probleme) oder externalisierendes Verhalten (z.B. aggressives Verhalten) zu verringern und prosoziales Verhalten zu fördern. Die bisherigen Forschungsbefunde fallen jedoch sehr unterschiedlich aus, wobei teilweise sogar unerwünschte Wirkungen durch außerunterrichtliche Bildungs- und Betreuungsangebote festgestellt wurden. Eine mögliche Erklärung für diese Unterschiede bieten die Interaktionen in den außerunterrichtlichen Bildungs- und Betreuungsangeboten. Allerdings fehlen empirische Untersuchungen zur Rolle der Gleichaltrigen und auch die Bedeutung der Interaktionsqualität zwischen dem Betreuungspersonal und den Schülerinnen und Schülern wurde bisher kaum berücksichtigt. Im Rahmen dieser Dissertation und des Forschungsprojekts EduCare-TaSe soll diesem Forschungsdesiderat begegnet werden.
\end{abstract}

Die vom Schweizerischen Nationalfonds finanzierte Längsschnittstudie EduCare-TaSe - Tagesschulen und Schulerfolg? untersuchte insgesamt 1'990 Primarschülerinnen und -schüler an 53 offenen Tagesschulen in der Deutschschweiz, wobei nur ein Teil der Schülerschaft das Tagesschulangebot nutzte. Der Untersuchungszeitraum erstreckte sich von Ende der 1. (T1) bis Ende der 2. (T2) Jahrgangsstufe. Dabei wurden Befragungen der Schul- und Tagesschulangebotsleitungen durchgeführt, um Informationen zum Ausbau des Tagesschulangebots, zu den zugrundeliegenden Motiven und zur Angebotsnutzung durch die einzelnen Schülerinnen und Schüler zu gewinnen. Die sozialen Kompetenzen der Schülerinnen und Schüler wurden zu beiden Messzeitpunkten durch die Lehrpersonen, anhand des „Strengths and Difficulties Questionnaire“ (SDQ), eingeschätzt. Die Interaktionen mit dem Betreuungspersonal wurden durch externe Beobachterinnen und Beobachter beurteilt. 
Mittels Mehrebenenanalysen wurde der generelle Einfluss der Nutzung von Tagesschulangeboten auf die Entwicklung von sozialen Kompetenzen überprüft, wobei Schülerinnen und Schüler mit einer dauerhaften Angebotsnutzung (d.h. Nutzung über ein ganzes Schuljahr) mit den Schülerinnen und Schülern ohne Angebotsnutzung verglichen wurden. Dabei zeigten sich hinsichtlich der Entwicklung von externalisierendem und internalisierendem Verhalten leicht verstärkende und somit unerwünschte Effekte durch eine dauerhafte Nutzung der Tagesschulangebote. Bezüglich prosozialem Verhalten zeigten sich hingegen keine Effekte.

Die gefundenen Effekte konnten zumindest teilweise durch die Interaktionen im Tagesschulangebot erklärt werden: Je mehr internalisierendes Verhalten von den Gleichaltrigen im Tagesschulangebot gezeigt wurde, desto unvorteilhafter wirkte sich die dauerhafte Angebotsnutzung auf die Entwicklung des internalisierenden Verhaltens aus. Ein analoger Moderationseffekt durch das Verhalten der Gleichaltrigen zeigte sich bezüglich der Entwicklung des externalisierenden Verhaltens, allerdings nur bei der dauerhaften Nutzung von Tagesschulangeboten mit einer unterdurchschnittlichen Interaktionsqualität mit dem Betreuungspersonal. Auch unabhängig vom Verhalten der Gleichaltrigen spielte die Interaktionsqualität mit dem Betreuungspersonal eine Rolle, wobei sich die dauerhafte Angebotsnutzung insbesondere bei einer niedrigen Interaktionsqualität verstärkend auf das externalisierende Verhalten auswirkte.

Die zukünftige Forschung zu außerunterrichtlichen Bildungs- und Betreuungsangeboten sollte vermehrt auf die Vorteile von Mehrebenenanalysen zurückgreifen und dabei mögliche Wirkungen durch die Gleichaltrigen in den Angeboten und durch die Betreuungsqualität berücksichtigen. Letztere zwei Punkte gelten auch für die Praxis an Tagesschulen, in der auf eine hohe Betreuungsqualität und eine gute Durchmischung der Schülerschaft im Tagesschulangebot geachtet werden sollte, damit es nicht zu unerwünschten Wirkungen kommt und die angestrebte Förderung von sozialen Kompetenzen erreicht werden kann. 


\section{Einleitung}

In den letzten Jahrzehnten gewann außerunterrichtliche Bildung und Betreuung im schulischen Rahmen zunehmend an Bedeutung. Dies gilt in besonderem Masse für den deutschsprachigen Raum: Während in Deutschland Ganztagsschulen vermehrt gesellschaftliche und politische Unterstützung fanden und stark ausgebaut wurden, zeigte sich in der Deutschschweiz mit den sogenannten Tagesschulen eine ähnliche Entwicklung. Unter anderem als bildungspolitische Maßnahme verstanden, wurde diese Zunahme ganztägiger Angebote von zahlreichen Erwartungen angetrieben oder zumindest begleitet. Eine davon bezieht sich auf eine verbesserte Förderung sozialer und emotionaler Kompetenzen ${ }^{1}$.

Ob diese Erwartung an Tages- und Ganztagsschulen tatsächlich erfüllt wird, wurde allerdings bisher kaum untersucht, wobei für die Schweiz lediglich eine Untersuchung zu Tagesschulen vorliegt (Schüpbach, 2010; Schüpbach, Ignaczewska \& Herzog, 2014). Ein ähnliches Forschungsdesiderat ist hinsichtlich Ganztagsschulen in Deutschland auszumachen, deren Wirkungen auf soziale Kompetenzen anhand von zwei Forschungsprojekten untersucht wurden, namentlich von der Studie ,peers in networks“ (PIN; Kanevski \& von Salisch, 2011) und der Studie zur Entwicklung von Ganztagsschulen, wobei für letztere mittlerweile Daten zur ersten (Fischer, Brümmer \& Kuhn, 2011; Fischer, Kuhn \& Züchner, 2011) und zur zweiten Erhebungswelle (StEG-P \& StEG-S; Sauerwein, Lossen, Theis, Rollett \& Fischer, 2018) vorliegen. Zusätzlich wird deshalb meist die umfangreiche Forschung zu sogenannten ,after-school“ Programmen herangezogen, die sich insbesondere in den USA als außerunterrichtliche Bildungsund Betreuungsangebote etabliert haben, und deren Wirkungen auf die Entwicklung sozialer

\footnotetext{
${ }^{1}$ Zur sozialen Kompetenz gehören auch emotionale Aspekte (vgl. Kapitel 3), weshalb einige Autorinnen und Autoren von „sozio-emotionaler Kompetenz“ sprechen. Zudem wird im Singular von der sozialen Kompetenz als Gesamtkonstrukt gesprochen, während mit dem Plural „soziale Kompetenzen“ die Mehrdimensionalität des Konstrukts verdeutlicht werden soll oder auf einzelne Dimensionen (z.B. aggressives Verhalten oder prosoziales Verhalten) Bezug genommen wird.
} 
Kompetenzen anhand von mehreren Meta-Analysen untersucht wurden (Durlak, Weissberg \& Pachan, 2010; Kremer, Maynard, Polanin, Vaughn \& Sarteschi, 2015; Taheri \& Welsh, 2016).

Sowohl im Hinblick auf Tages- und Ganztagsschulen als auch unter Berücksichtigung der Befunde zu after-school Programmen ist insbesondere die Heterogenität in den Forschungsergebnissen augenfällig. Deshalb wurden wiederholt Qualitätsanforderungen an außerunterrichtliche Bildungs- und Betreuungsangebote diskutiert, allerdings häufig ohne konkrete empirische Befunde. Ein Aspekt, der dabei besonders vernachlässigt wurde, stellt die Rolle der Gleichaltrigen dar. Dies ist nicht unproblematisch, könnten sich doch gerade Mitschülerinnen und Mitschüler für ungewollte Wirkungen, sogenannte ,,peer-contagion“ (Dishion \& Tipsord, 2011), verantwortlich zeigen. Auch zum Einfluss der Betreuungsqualität, respektive der Interaktionsqualität des Betreuungspersonals (bezogen auf die alltäglichen Interaktionen des Betreuungspersonals mit den Schülerinngen und Schülern), gibt es erst wenig Forschung (Fischer, Kuhn et al., 2011; Pierce, Bolt \& Vandell, 2010; Wade, 2015). Dabei fehlen bisher insbesondere Längsschnittstudien. Nebst der generellen Wirkung von Tagesschulangeboten auf die Entwicklung von sozialen Kompetenzen geht diese Dissertation deshalb der Frage nach, welche Rolle die alltäglichen Interaktionen mit den Gleichaltrigen und mit dem Betreuungspersonal spielen.

Dazu wird im zweiten Kapitel der Synopse genauer ausgeführt, was unter Tagesschulen verstanden wird, und wie diese in der gegenwärtigen Bildungslandschaft einzuordnen sind. Darauf folgt im dritten Kapitel eine Definition von sozialer Kompetenz, bevor sich das vierte Kapitel mit der Frage beschäftigt, wieso sich Tagesschulangebote auf die Entwicklung von sozialen Kompetenzen auswirken könnten. Kernstück der Synopse bildet die Darstellung der bisherigen Forschungsbefunde im fünften Kapitel, wobei ein besonderes Augenmerk auf die Forschungsdesigns und die jeweils angewandten statistischen Verfahren gelegt wird. Da die Dissertation im Rahmen des Forschungsprojektes „EduCare TaSe - Tagesschulen und Schulerfolg?“ durchgeführt wurde und sich auf entsprechende Daten stützt, wird im sechsten Kapitel kurz auf das 
Forschungsprojekt und das dabei angewendete methodische und statistische Design eingegangen. Im siebten Kapitel folgt ein Abriss über die vier Manuskripte und die damit verbundenen Ergebnisse, die im Rahmen dieser Dissertation publiziert oder eingereicht wurden. Den Abschluss bildet das achte Kapitel mit einer Integration der Ergebnisse im Hinblick auf den bisherigen Forschungsstand und den gewonnenen Implikationen für die weitere Forschung als auch für die Praxis. 


\section{Tagesschulen in der Deutschschweiz}

Gemäß Bundesverfassung sind in der Schweiz die 26 Kantone für das Schulwesen zuständig (SKBF, 2007), was eine gewisse Heterogenität in der konkreten Ausgestaltung der Lehrpläne, der Klassenstufen, oder auch des Schulalltags bedeutet. In Bezug auf letzteren herrschte gegen Ende des 19. Jahrhunderts eine traditionelle „Ganztagsschulorganisation“ vor, mit eher kurzen Vor- und Nachmittagsblöcken, deren Anfangs- und Schlusszeiten täglich variierten und die keine Mittagsbetreuung oder -verpflegung beinhalteten (Schüpbach, 2010). Damit war der Schulalltag am traditionellen Familien- und Erwerbsmodell ausgerichtet, bei dem primär die Mutter für die Kinderbetreuung zuständig ist, während der Vater einer Vollzeitbeschäftigung nachgeht, und erforderte eine große Flexibilität von Seite der Eltern. Der demographische Wandel und weitere gesellschaftliche Entwicklungen, namentlich die veränderten familialen Lebensformen, eine verminderte Familiengröße sowie eine zunehmende Bildungs- und Erwerbstätigkeit der Frauen, stärkte den Wunsch nach außerfamilialen Betreuungsformen und führte zu Konflikten mit der gängigen Schulorganisation (Schüpbach, 2010).

Eine Reaktion auf die gesellschaftlichen Veränderungen stellte die Einführung des Blockzeitenunterrichts dar, bei dem die Schülerinnen und Schüler an fünf Vormittagen pro Woche wenigstens zu dreieinhalb Stunden (oder während vier Lektionen) und an einem bis vier Nachmittagen unter der Obhut der Schule stehen (EDK, 2015). Dies führte insbesondere am Vormittag zu einheitlicheren Unterrichtszeiten und einer erleichterten Vereinbarkeit von Beruf und Familie. Im Schuljahr 2014/2015 verfügten bereits rund zwei Drittel der Schweizer Kantone über einen nahezu flächendeckenden Blockzeitenunterricht (EDK, 2015), womit der Grundstein für weitere Bildungs- und Betreuungsangebote vor Unterrichtsbeginn, über Mittag sowie in der unterrichtsfreien Zeit am Nachmittag gelegt wurde. 
Ein mögliches Gefäß für diese außerunterrichtlichen Angebote stellen Tagesschulen dar, welche durch die Schweizerische Konferenz der kantonalen Erziehungsdirektoren (EDK) als Schulen mit ganztägigen Betreuungsangeboten (inklusive Mittagsverpflegung) an mehreren Tagen pro Woche definiert wurden (EDK, 2015). Die Angebote an Tagesschulen beinhalten üblicherweise das Mittagessen, ein beaufsichtigtes Programm am Nachmittag (z.B. Aktivitäten, freies Spiel oder Hausaufgabenbetreuung) und etwas seltener eine Betreuung vor Schulbeginn. Während der Unterricht und die Angebote an einigen Tagesschulen mit Ausnahme sogenannter Auffangzeiten für alle Schülerinnen und Schüler verpflichtend sind (gebundene Tagesschulen), bieten die meisten Tagesschulen neben dem Unterricht modular aufgebaute Angebote an, die freiwillig genutzt werden können (offene Tagesschule) (Schüpbach, 2010).

Als Reaktion auf die ersten PISA-Ergebnisse, die insbesondere bezüglich Leseleistungen und Herkunftseffekten einen Handlungsbedarf aufzeigten, wurde die Einführung von Tagesschulen durch die nationale PISA-Projektleitung als mögliche Reaktion diskutiert (Coradi Vellacott, Hollenweger, Nicolet \& Wolter, 2003). Auch im HarmoS-Konkordat, das eine Harmonisierung des Schulwesens in Bezug auf strukturelle und inhaltliche Aspekte anstrebt und dem sich mittlerweile 15 Kantone angeschlossen haben, wird ein „,bedarfsgerechtes Angebot an Tagesstrukturen“ (SKBF, 2007, S. 60) gefordert. Zudem trat 2003 ein Bundesgesetz über Finanzhilfen für familienergänzende Kinderbetreuung in Kraft (Schüpbach, 2018). Als Folge dieser Anstrengungen ist denn auch ein Anstieg von Tagesschulen zu beobachten (Stern et al., 2013), allerdings sind diese nach wie vor wenig verbreitet und beschränken sich meist auf einige wenige Schulen pro Kanton (EDK, 2015). Genaue Angaben zur Verbreitung und Nutzung von Tagesschulen, wie sie für Ganztagsschulen in Deutschland existieren, fehlen bislang. 


\section{Soziale Kompetenz}

Eine Erwartung, die in den Begründungslinien von Tages- und Ganztagsschulen sowie in den kantonalen Leitfäden und Handreichungen zu Tagesschulen immer wieder auftaucht, bezieht sich auf die Entwicklung der sozialen Kompetenz. Grob formuliert bedeutet soziale Kompetenz eine „effectiveness in interactions“ (Rose-Krasnor, 1997, S. 112), also eine Effektivität in Interaktionen. Kanning (2002) definiert soziale Kompetenz als „Gesamtheit des Wissens, der Fähigkeiten und Fertigkeiten einer Person, welche die Qualität eigenen Sozialverhaltens [...] fördert“ (S. 155). Somit wird soziale Kompetenz als mehrdimensionales Konstrukt betrachtet, das sowohl konkrete Verhaltensweisen, als auch Wahrnehmungen, Kognitionen, Motive oder Emotionen umfasst (Kanning, 2002; Rose-Krasnor, 1997). Entsprechend wird auch im Plural von sozialen Kompetenzen oder von sozio-emotionalen Kompetenzen gesprochen (Kanning, 2002; Rose-Krasnor \& Denham, 2009). Ein Defizit an sozialer Kompetenz äußert sich in Verhaltensauffälligkeiten, namentlich in störendem oder aggressivem Verhalten, einer mangelhaften Konzentrationsfähigkeit, Problemen mit Gleichaltrigen oder auch emotionalen Problemen. Dabei wird häufig zwischen internalisierenden und externalisierenden Verhaltensweisen unterschieden (Fröhlich-Gildhoff, 2013; Liu, 2004): Internalisierendes Verhalten ist durch „Rückzug und Selbsteinschränkung“ gekennzeichnet, während externalisierendes Verhalten ein „Agieren nach außen (z. B. ein besonderes Maß an Aggressivität)“ darstellt (Fröhlich-Gildhoff, 2013, S. 39). Einen Kontrastpunkt zu diesen unerwünschten Verhaltensweisen setzt prosoziales Verhalten. Dieses wird aber nicht einfach als Gegenpol zu Verhaltensauffälligkeiten betrachtet, sondern bezieht sich auf eine besonders stark ausgeprägte Berücksichtigung fremder Interessen (Kanning, 2002). Eine faktorenanalytische Analyse von Goodman, Lamping und Ploubidis (2010) konnte prosoziales, internalisierendes und externalisierendes Verhalten als eigenständige Konstrukte bestätigen. 
Internalisierendes, externalisierendes und prosoziales Verhalten wirken sich auch auf die weitere Entwicklung aus: So erhöht internalisierendes Verhalten im Schulalter die Wahrscheinlichkeit, ein Opfer von Gewalt durch Gleichaltrige zu werden (Reijntjes, Kamphuis, Prinzie \& Telch, 2010). Externalisierendes Verhalten während den ersten Schuljahren führt vermehrt zu Suchtproblemen (Fergusson, Horwood \& Ridder, 2007) und wirkt sich negativ auf den Schulerfolg aus, wobei die schulische Leistung abnimmt und seltener ein Hochschulabschluss erreicht wird (McLeod \& Kaiser, 2004; Metsäpelto et al., 2015). Sowohl internalisierendes als auch externalisierendes Verhalten wurden mit erhöhten Entzündungswerden in Zusammenhang gebracht (Slopen, Kubzansky \& Koenen, 2013) und dürften sich somit auch ungünstig auf die psychische Gesundheit auswirken. Im Gegensatz dazu fördert prosoziales Verhalten positive Emotionen (Nelson, Layous, Cole \& Lyubomirsky, 2016) und kann einer Viktimisierung durch Gleichaltrige entgegenwirken (Leadbeater, Thompson \& Sukhawathanakul, 2016). Bereits geringe Ausprägungen von internalisierendem und externalisierendem Verhalten, oder ein Mangel an prosozialem Verhalten, begünstigen die Entwicklung bestimmter psychischer Störungen (Goodman et al., 2010). 


\section{Fördert die Teilnahme am Tagesschulangebot soziale Kompetenzen?}

Eines der Leitziele von Ganztagsschulen in Deutschland bezieht sich auf „Soziales Lernen über verschiedene Altersgruppen hinweg durch Angebote, die das Leben in Gemeinschaft, respektvollen Umgang miteinander und soziale Kompetenz fördern“ (Bundesministerium für Bildung und Forschung, 2003, S. 6). Auch in zahlreichen Leitlinien von Deutschschweizer Tagesschulen wurde soziales Lernen als pädagogischer Grundsatz und als Ziel von Tagesschulangeboten festgehalten, und wird - zusammen mit interkulturellem Lernen - sogar noch etwas häufiger genannt als die Kompetenzförderung und Persönlichkeitsentwicklung im generellen (Schüpbach, Andrey \& Arpagaus, 2018).

Aber wieso geht man überhaupt davon aus, dass der Besuch von Tagesschulangeboten soziale Kompetenzen fördern könnte? Eine zentrale Annahme bezieht sich darauf, dass bei einer Nutzung von außerunterrichtlichen Bildungs- und Betreuungsangeboten die unstrukturierte und unbeaufsichtigte Freizeit reduziert wird (Taheri \& Welsh, 2016). Während Schülerinnen und Schüler im Tagesschulangebot sind, so die Annahme, kann der Einfluss der Gleichaltrigen besser kontrolliert werden und sinnvolle, soziale Tätigkeiten können gefördert werden. Aeberli und Binder (2005) sprechen in ihrem Leitfaden zu Tagesschulen denn auch von einem pädagogisch und zeitlich erweiterten Förderrahmen, der den Kindern mehr Zeit und Gelegenheiten bietet, um soziale Fertigkeiten zu üben. Als konkrete Beispiele nennen sie „die gegenseitige Rücksichtnahme, das Einhalten von Regeln in der Gruppe, am Tisch oder beim Spiel oder auch Regeln im Gespräch“(Aeberli \& Binder, 2005). Eine weitere Argumentationslinie betont die soziale Integration und eine Erweiterung des sozialen Netzwerks (Coradi Vellacott et al., 2003). Gerade bei Schülerinnen und Schülern, die über weniger familiale und soziale Ressourcen verfügen, könnte der Besuch des Tagesschulangebots zu einer Integration in das schulische Milieu und zu neuen oder intensiveren Kontakten mit Mitschülerinnen und Mitschülern oder auch mit dem Lehr- und Betreuungspersonal führen. 
Die Erwartung an eine Förderung von sozialen Kompetenzen durch die Teilnahme an außerunterrichtlichen Bildungs- und Betreuungsangeboten ist auch im „Modell der Bildungsqualität außerunterrichtlicher Angebote in der Ganztagsschule“ dargestellt (Abbildung 1). Das Modell wurde ursprünglich für after-school Programme formuliert (Miller, 2003) und später an den Deutschsprachigen Kontext angepasst (Radisch, Stecher, Fischer \& Klieme, 2008; Stecher, Radisch, Fischer \& Klieme, 2007). Nebst den Wirkungen auf Leistungen und Schulerfolg geht das Modell auch von erzieherischen Wirkungen außerunterrichtlicher Angebote aus, darunter soziales Lernen. Beeinflusst wird die Wirkungsebene (Felder 7 und 8) einerseits durch eine Prozessebene (Felder 5 und 6) und andererseits durch eine Input- und Kontextebene (Felder 1-4). Zudem hält das Modell fest, dass es auch eine Wechselwirkung zwischen erzieherischen Wirkungen und der Leistungsentwicklung gibt. Als Input- und Kontextmerkmale gelten Merkmale der Schule und spezifisch auf den außerunterrichtlichen Teil bezogene Merkmale, aber auch

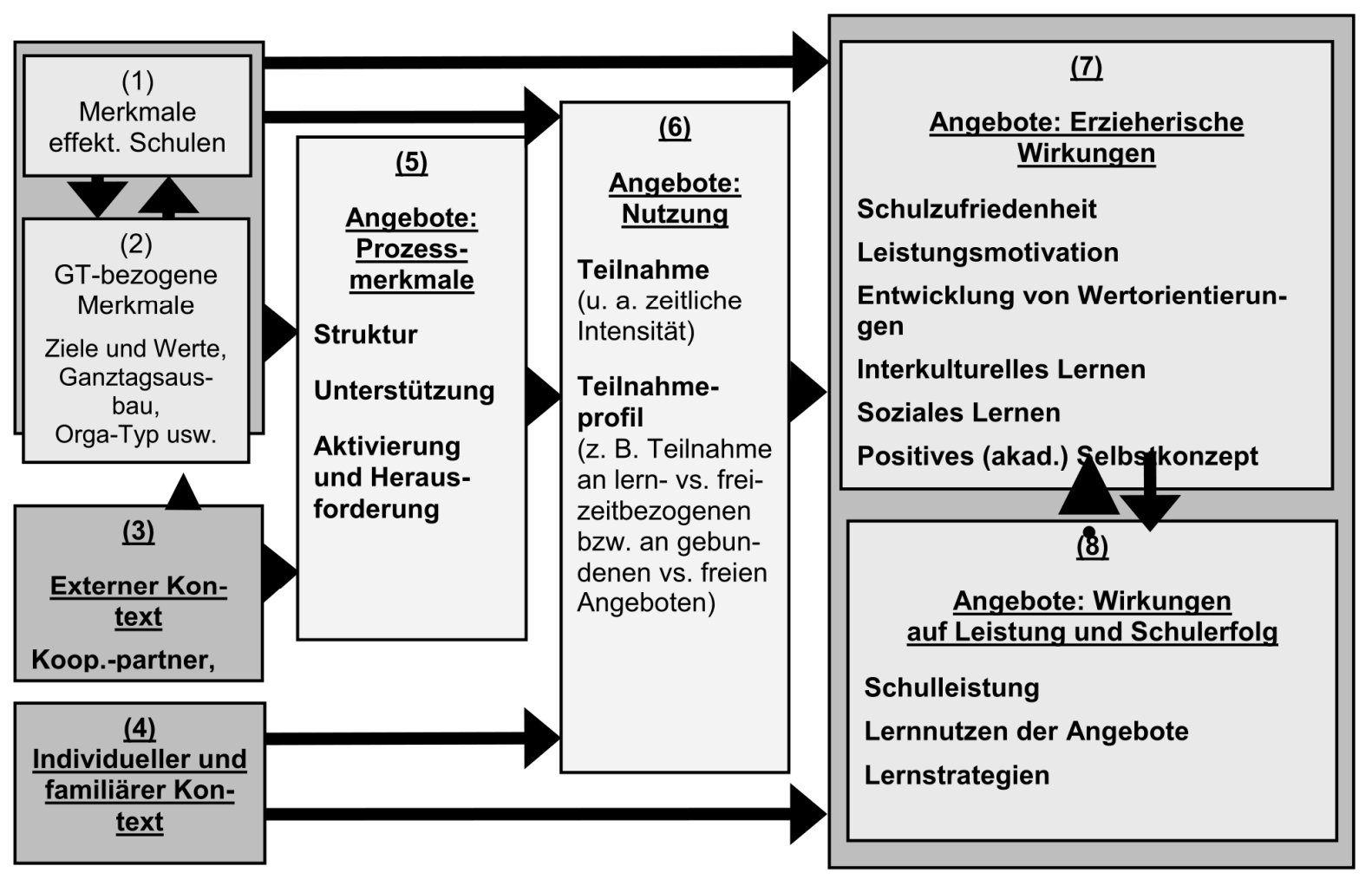

Abb. 1: Modell der Bildungsqualität außerunterrichtlicher Angebote in der Ganztagsschule (Radisch et al., 2008) 
der externe Kontext (z.B. mögliche außerschulische Kooperationspartner) sowie der individuelle und familiäre Kontext.

Zentraler Teil des Modells bildet die Prozessebene, welche auch für die vorliegende Untersuchung besonders relevant ist, wobei zwischen der Angebotsqualität und der Angebotsnutzung unterschieden wird. So geht das Modell davon aus, dass sich die außerunterrichtlichen Angebote nicht direkt auf die Persönlichkeits- und Leistungsentwicklung auswirken, sondern durch die Ausgestaltung dieser Angebote sowie durch die individuelle Nutzung der einzelnen Schülerinnen und Schüler moderiert und mediiert wird. Hinsichtlich der Angebotsqualität bezieht sich das Modell auf die unter anderem von Klieme (2006) genannten Basisdimensionen der Unterrichtsqualität, die von Radisch et al. (2008) auf den außerunterrichtlichen Kontext adaptiert wurden:

- „Verlässlichkeit, Sicherheit und Strukturiertheit der Lernumgebung, ablesbar u.a. an angemessenen und konsistenten Regeln und an klarer, altersangemessener Führung durch Erwachsene [Strukturdimension];

- akzeptierende und respektvolle Beziehungen zu anderen Kindern und Jugendlichen und zu Erwachsenen, die ein Gefühl der Zugehörigkeit geben, positive soziale Normen vermitteln und persönliches Wachstum unterstützen [Unterstützungs- und Orientierungsdimension];

- Herausforderungen und Gelegenheiten zur Entfaltung der körperlichen, intellektuellen, emotionalen und sozialen Fähigkeiten [Herausforderungs- und Aktivierungsdimension].“(Radisch et al., 2008, S. 278f)

Hierbei ist wichtig zu beachten, dass sich gerade die ersten beiden Dimensionen ganz konkret auf die Interaktionen und Beziehungen des Betreuungspersonals mit den Schülerinnen und 
Schülern beziehen, und in der zweiten Dimension zusätzlich die Bedeutung der Mitschülerinnen und Mitschüler betont wird. Für die vorliegende Untersuchung bedeutet dies, dass insbesondere dann positive Wirkungen einer Angebotsnutzung auf soziale Kompetenzen zu erwarten sind, wenn die Interaktionen mit dem Betreuungspersonal und den Gleichaltrigen von hoher Qualität sind.

Nebst der Bedeutung der Angebotsqualität betont das Modell, dass die Angebote auch entsprechend genutzt werden müssen, damit sie ihre Wirkungen entfalten können. So müssen die Angebote überhaupt einmal genutzt werden (generelle Nutzung), zudem dürfte der Teilnahmedauer (über welchen Zeitraum erstreckt sich die Nutzung?), der Teilnahmeintensität (wie häufig wird das Angebot über einen bestimmten Zeitraum genutzt?) sowie der Teilnahmebreite (an wie vielen Aktivitäten nimmt eine Schülerin oder ein Schüler teil?) eine wichtige Rolle zukommen (Radisch et al., 2008). Roth, Malone und Brooks-Gunn (2010) nennen zusätzlich die gesamthafte Exposition (,,total exposure“; wie häufig wird das Angebot über den gesamten Zeitraum genutzt?) sowie die persönliche Involviertheit der Schülerinnen und Schüler (,engagement") als wichtige Komponenten der Angebotsnutzung. 


\section{Forschungsstand}

Obwohl die Erwartung an eine verbesserte Förderung von sozialen Kompetenzen durch Tagesund Ganztagsschulen immer wieder anzutreffen ist, gibt es dazu bislang erst wenige und teils widersprüchliche Befunde. Der Mangel an Forschungsergebnissen dürfte insbesondere daran liegen, dass sowohl Tages- als auch Ganztagsschulen eine neuere Entwicklung darstellen und die meiste Forschung dazu - abgesehen von Einzelfallanalysen - innerhalb der letzten 15 Jahre anzusiedeln ist. Aus diesem Grund wird zusätzlich die deutlich umfangreichere Forschung zu after-school Programmen herangezogen, wozu es mittlerweile auch mehrere Metaanalysen gibt. Diese Programme sind insbesondere in den USA weit verbreitet und weisen einige Gemeinsamkeiten zu Tages- und Ganztagsschulen auf, indem sie eine strukturierte und beaufsichtigte Umgebung außerhalb des Unterrichts bieten, wobei verschiedenste soziale und lernförderliche Aktivitäten angeboten werden. Wie der Name schon sagt, handelt es sich dabei allerdings um eine Vielzahl unterschiedlicher Programme, in denen häufig auf spezifische Entwicklungsaspekte eingewirkt werden soll - manchmal mit einem Fokus auf ethnische Minderheiten oder auf Kinder mit einem niedrigen sozioökonomischen Status - und die nicht als direkter Bestandteil der Schule verstanden werden (Kremer et al., 2015). Trotz dieser Unterschiede und wenn man bedenkt, dass auch Tages- und Ganztagsschulen starke Unterschiede hinsichtlich der Ziele und Aktivitäten aufweisen können, bietet die umfangreiche Forschung zu after-school Programmen wertvolle Informationen zu möglichen Wirkungen von Tages- und Ganztagsschulen auf die Entwicklung von sozialen Kompetenzen.

Die Forschung zu außerunterrichtlichen Bildungs- und Betreuungsangeboten ist mit einigen methodischen und statistischen Herausforderungen behaftet: Erstens beruht die unabhängige Variable - die Teilnahme am außerunterrichtlichen Angebot - meist auf einer Selbstselektion. Dies nicht nur durch die Schulen, welche über das entsprechende Angebot verfügen müssen, sondern auch durch die einzelnen Schülerinnen und Schülern, welche die Angebote nutzen. 
Zweitens handelt es sich um eine geclusterte Stichprobe mit einer Mehrebenenstruktur, mit den Schülerinnen und Schülern als Teil einer Klasse und den Klassen als Teil einer Schule. Angesichts dieser Herausforderungen beschränkt sich die Darstellung des bisherigen Forschungsstandes nicht nur auf die Ergebnisse, sondern versucht auch zu ergründen, inwieweit die Selbstselektion und die Mehrebenenstruktur bei den einzelnen Studien und Metaanalysen berücksichtigt wurden.

Dazu wurde eine Literaturrecherche in einschlägigen Datenbanken (FIS-Bildung, PsycINFO, PSYNDEX) durchgeführt, wobei gezielt nach Studien zu Tages- und Ganztagsschulen, sowie nach Meta-Analysen zu after-school Programmen, gesucht wurde. Zentrales Kriterium war, dass Schülerinnen und Schüler mit einer Nutzung der außerunterrichtlichen Angebote mit Schülerinnen und Schülern ohne Nutzung verglichen wurden, wobei mindestens ein Aspekt von sozialer Kompetenz als abhängige Variable untersucht werden musste. Zudem wurden nur Studien und Metaanalysen berïcksichtigt, die seit 2006 publiziert wurden. Diese Arbeiten werden im Folgenden präsentiert, wobei jeweils auf die Stichprobe, die Ergebnisse sowie auf das methodische Design und das statistische Vorgehen - insbesondere im Hinblick auf mögliche Selektionseffekte und die Mehrebenenstruktur - eingegangen wird. Sofern Moderatoren untersucht wurden, werden die entsprechenden Ergebnisse ebenfalls berichtet. Am Schluss des Kapitels folgt ein Fazit zum bisherigen Forschungsstand.

\section{EduCare-Studie (Offene und gebundene Tagesschulen in der Deutschschweiz)}

Bisher hat lediglich eine Studie den Einfluss der Angebotsnutzung an Deutschschweizer Tagesschulen auf die Entwicklung sozialer Kompetenzen untersucht, nämlich die EduCare-Studie (Schüpbach, 2010; Schüpbach et al., 2014). Die Stichprobe bestand dabei aus Grundschülerinnen und -schülern, die im Schuljahr 2006/2007 die erste Primarschulklasse besuchten und zum Teil offene, zum Teil gebundene und zum Teil keine Tagesschulangebote nutzten, oder Schulen ohne Tagesschulangebot besuchten. Obwohl die berücksichtigten Schulen zufällig aus einem 
Pool von Schulen mit vergleichbaren sozio-demographischen Merkmalen ausgewählt wurden und auch auf Klassenebene eine Zufallsauswahl von durchschnittlich neun Kinder pro Klasse erfolgte, können Selektionseffekte in Abhängigkeit der Bereitstellung eines Tagesschulangebots (Ebene der Schule) oder der Teilnahme an demselben (Ebene der Schülerinnen und Schüler) nicht ausgeschlossen werden.

Schüpbach et al. (2014) untersuchten die Entwicklung von prosozialem Verhalten und sozioemotionalen Verhaltensstärken von Ende der ersten bis Ende der dritten Klassenstufe anhand von linearen Wachstumskurvenmodellen mit drei Messzeitpunkten, wobei die Schulzugehörigkeit bei der Schätzung der Standardfehler berücksichtigt wurde. Die Skala zu sozio-emotionalen Verhaltensstärken umfasste sowohl Items zu Hyperaktivität als auch zu emotionalen Problemen und wurde, ebenso wie die Skala zu prosozialem Verhalten, durch die Eltern der Schülerinnen und Schüler eingeschätzt. Von den 235 untersuchten Schülerinnen nutzten 201 Schülerinnen und Schüler kein Tagesschulangebot und wurden mit den 34 Schülerinnen und Schülern verglichen, welche die Angebote an mindestens drei Tagen pro Woche zu mindestens 7.5 Stunden nutzten. Die Analysen, welche hinsichtlich Geschlecht, IQ und familialen Ressourcen kontrollierten, zeigten keinen signifikanten Einfluss der Angebotsnutzung auf die Entwicklung von prosozialem Verhalten oder von sozio-emotionalen Verhaltensstärken (Schüpbach et al., 2014).

Zusätzlich wurde bei EduCare anhand von linearen Regressionsmodellen untersucht, ob die Breite und Qualität der Aktivitäten, respektive die Strukturierung der pädagogischen Arbeit, die Entwicklung der 34 Schülerinnen und Schüler mit Angebotsnutzung beeinflusst. Hinsichtlich der Entwicklung des prosozialen Verhaltens zeigte die Breite und Qualität der Aktivitäten einen signifikanten Einfluss, hinsichtlich der Entwicklung der sozio-emotionalen Verhaltensstärken wurde ein signifikanter Effekt der Strukturierung der pädagogischen Arbeit aufgezeigt. 


\section{PIN-Studie (gebundene Ganztagsschulen in Deutschland)}

Im Rahmen der Studie „Peers in Netzwerken“ (PIN) untersuchten Kanevski und von Salisch (2011) die Entwicklung von sozialen Kompetenzen im Verlauf der siebten Jahrgangsstufe (Herbst 2008 bis Sommer 2009), wobei Schülerinnen und Schüler an gebundenen Ganztagsschulen mit Schülerinnen und Schülern an Halbtagsschulen verglichen wurden. Um Selektionseffekte zu verhindern wurden ausschließlich Schulen mit einem relativ niedrigen sozioökonomischen Status, mit einem geringen Migrantenanteil und aus dem urbanen Raum berücksichtigt. Darüberhinausgehende Selektionseffekt hinsichtlich der Schülerschaft oder Merkmalen der Schulen können allerdings nicht ausgeschlossen werden. Da jeweils alle Schülerinnen und Schüler einer Schule die Ganztagsangebote nutzen oder nicht nutzen, sind Selektionseffekte auf der Individualebene unwahrscheinlich.

Die verschiedenen sozialen Kompetenzen wurden mittels Selbsteinschätzungen der Schülerinnen und Schüler erhoben, mit Ausnahme des körperlich aggressiven Verhaltens, welches zusätzlich durch die Mitschüler eingeschätzt wurde, und des prosozialen Verhaltens, welches auf einem Urteil der Lehrkräfte basierte. Für die Analysen wurden multivariate Varianzanalysen mit Messwiederholung gerechnet, wobei Ausgangsniveau und Geschlecht kontrolliert wurden. Je nach abhängiger Variable standen zwischen 307 und 380 Schülerinnen und Schüler für die Analysen zur Verfügung, wobei etwa 48 Prozent eine Ganztagsschule besuchten.

Kein Unterschied in der Entwicklung von Schülerinnen und Schülern an Ganz- oder Halbtagsschulen zeigte sich für das prosoziale Verhalten (Lehrkrafturteil), das körperlich aggressive Verhalten (Selbsteinschätzung), die Klarheit fremder oder eigener Emotionen sowie die Selbstabwertung als Mittel zur Selbstregulation. Eine vorteilhaftere Entwicklung zeigten die Ganztagsschülerinnen und -schüler hinsichtlich der emotionalen Fremdaufmerksamkeit (d.h. sie schenkten den Gefühlen anderer Menschen zunehmend mehr Aufmerksamkeit) und hinsichtlich der Aufmerksamkeitslenkung als Mittel zur Selbstregulation. Zudem nahm das körperlich 
aggressive Verhalten (Fremdurteil) bei den Knaben an Ganztagsschulen im Vergleich zu Halbtagsschülern leicht ab. Allerdings kam es bei den Mädchen zu einem gegenteiligen Effekt, wobei sich die Schülerinnen in den Halbtagsschulen vorteilhafter entwickelten als an Ganztagsschulen. Ein weiterer geschlechtsspezifischer Effekt zeigte sich hinsichtlich der emotionalen Selbstaufmerksamkeit, wobei nur Knaben vom Besuch der Ganztagsschule profitieren konnten.

\section{StEG - 1. Förderphase (offene und gebundene Ganztagsschulen in Deutschland)}

Die bisher größte Studie im Deutschsprachigen Raum stellt die „Studie zur Entwicklung von Ganztagsschulen (StEG)“ dar (Fischer, Brümmer et al., 2011; Fischer, Kuhn et al., 2011). In einer ersten Förderphase wurde eine für Deutschland repräsentative Stichprobe von Schülerinnen und Schülern rekrutiert, wobei bei einem Teil der Schülerschaft die Entwicklung von der fünften (Jahr 2005) über die siebte (Jahr 2007) bis in die neunte Jahrgangsstufe (Jahr 2009) untersucht wurde. Die Schülerinnen und Schüler stammen aus Schulen mit oder ohne außerunterrichtlichen Angeboten, wobei letztere sowohl der offenen als auch der gebundenen Form angehören können. Folglich sind Selektionseffekte hinsichtlich der Verfügbarkeit oder der Nutzung vom Ganztagsangebot - wie bereits bei Schüpbach et al. (2014) - nicht auszuschließen. Verglichen wurden Schülerinnen und Schüler mit einer dauerhaften Teilnahme am außerunterrichtlichen Angebot (Teilnahme zu mindestens zwei Messzeitpunkten) mit den Schülerinnen und Schülern, welche höchstens zu einem Messzeitpunkt am Angebot teilnahmen.

Als abhängige Variablen wurden das problematische Verhalten im Schulalltag, Gewalt und Absentismus, soziale Verantwortungsübernahme und schulisches Wohlbefinden - jeweils aus Perspektive der Schülerinnen und Schüler - erhoben (Fischer, Brümmer et al., 2011; Fischer, Kuhn et al., 2011). Die Analysen erfolgten mittels linearer Wachstumskurvenmodellen, unter Berücksichtigung der drei Messzeitpunkte und unter Kontrolle von sozioökonomischem Status, Migrationshintergrund, Geschlecht, Schulform (Gymnasium vs. andere) und Bindungsgrad (offene 
Ganztagsschule vs. andere). Auf Mehrebenenanalysen wurde aufgrund geringer Varianzen zwischen den Schulen verzichtet (Fischer, Kuhn et al., 2011), oder sie wurden - im Fall des schulischen Wohlbefindens - zwar durchgeführt aber nicht berichtet (Fischer, Brümmer et al., 2011). Informationen zur Varianz zwischen den Klassen liegen hingegen nicht vor. In den berichteten Analysen, welche insgesamt 6‘853 Schülerinnen und Schüler (davon 3‘163 Schülerinnen und Schüler mit einer dauerhaften Angebotsnutzung) umfassten, wurde schließlich eine Korrektur des Standardfehlers aufgrund der Schulzugehörigkeit vorgenommen.

Die Ergebnisse zeigten dahingehend einen wünschenswerten Einfluss der dauerhaften Angebotsnutzung, dass problematisches Verhalten im Schulalltag, ebenso wie Gewalt und Absentismus, reduziert werden konnten. Hinsichtlich der sozialen Verantwortungsübernahme und dem schulischen Wohlbefinden zeigten sich hingegen keine Effekte. Über diese generellen Wirkungen hinaus wurde anhand der Schülerinnen und Schüler mit einer dauerhaften Angebotsnutzung untersucht, ob die verschiedenen Merkmale sozialer Kompetenz mit der von den Schülerinnen und Schülern wahrgenommenen Prozessqualität sowie mit den von den Schülerinnen und Schülern eingeschätzten Beziehungen zum Betreuungspersonal zusammenhängen (Fischer, Brümmer et al., 2011; Fischer, Kuhn et al., 2011). Dabei zeigte die Prozessqualität zu sämtlichen Messzeitpunkten einen signifikant positiven Zusammenhang zum schulischen Wohlbefinden und zur sozialen Verantwortungsübernahme. Zudem zeigte die Schüler-Betreuer-Beziehung zu sämtlichen Messzeitpunkten einen signifikant negativen Zusammenhang zu problematischem Verhalten im Schulalltag sowie zu Gewalt und Absentismus und einen signifikant positiven Zusammenhang zum schulischen Wohlbefinden. Leider liegen keine prädiktiven Befunde zum Einfluss auf die Entwicklung der sozialen Kompetenzen vor, um die Bedeutung dieser wahrgenommenen Angebotsmerkmale zusätzlich zu untermauern. Hinsichtlich der sozialen Verantwortungsübernahme wurde zusätzlich anhand von Cross-Lagged-Panel-Modellen untersucht, ob diese durch die Möglichkeiten zur Mitbestimmung im Ganztagsangebot beeinflusst wird 
(Kuhn, Fischer \& Schoreit, 2016). Dabei zeigten sich die wahrgenommenen Möglichkeiten zur Mitbestimmung in der 5. Jahrgangsstufe als prädiktiv für die soziale Verantwortungsübernahme in der 7. Jahrgangsstufe, was allerdings nur für Knaben der Fall war. Die Mitbestimmungsmöglichkeiten in der 7. Jahrgangsstufe hatten hingegen keinen Einfluss auf die Verantwortungsübernahme in der 9. Jahrgangsstufe.

\section{StEG - 2. Förderphase (offene und gebundene Ganztagsschulen in Deutschland)}

In der 2. Förderphase von StEG wurde die Entwicklung von prosozialem Verhalten anhand von zwei Teilstudien untersucht (Sauerwein et al., 2018): StEG-P fokussierte das Ende der Primarstufe, wobei Schülerinnen und Schüler von Mitte der dritten bis Ende der vierten Jahrgangsstufe befragt wurden. StEG-S fokussierte den Anfang der Sekundarstufe I, wobei Schülerinnen und Schüler von Anfang bis Ende der fünften Jahrgangsstufe (Schuljahr 2013/2014) befragt wurden. Für die Stichprobe wurden ausschließlich gebundene oder offene Ganztagsschulen rekrutiert, wodurch die Teilnahme für einige Schülerinnen und Schüler freiwillig war, während andere dazu verpflichtet wurden. Entsprechend sind auch hier Selektionseffekte auf Schul- und Individualebene möglich. Anders als bei den zuvor besprochenen Analysen steht hier nicht die Teilnahme am Ganztagsangebot im generellen, sondern die Teilnahme an spezifischen Angeboten in den Bereichen Medien oder soziales Lernen im Fokus.

Um längsschnittliche Effekte der Teilnahme an solchen Angeboten auf die Entwicklung des per Selbsteinschätzung erhobenen prosozialen Verhaltens zu prüfen, wurden lineare Regressionsanalysen gerechnet, wobei die Schulzugehörigkeit bei der Schätzung der Standardfehler berücksichtigt wurde. Die genaue Stichprobengröße für diese Analysen ist nicht ersichtlich, dürfte aber sowohl für StEG-P als auch für StEG-S bei jeweils etwa 2`000 Schülerinnen und Schülern liegen. Ganztagsangebote im Bereich Medien, respektive soziales Lernen, wurden zu jedem Messzeitpunkt von etwa 10-20 Prozent der Schülerschaft besucht (Sauerwein et al., 2018). Un- 
ter Kontrolle des Ausgangsniveaus und weiterer Variablen (sozioökonomischer Status, Migrationshintergrund, Geschlecht und Schulform) hatte die Nutzung von Angeboten aus den Bereichen Medien oder soziales Lernen keinen Einfluss auf das prosoziale Verhalten am Ende der vierten Jahrgangsstufe (StEG-P) respektive am Ende der fünften Jahrgangsstufe (StEG-S).

Innerhalb der Schülerinnen und Schüler mit einer Nutzung dieser spezifischen Angebote wurde zudem erneut der Zusammenhang zwischen der selbsteingeschätzten Angebotsqualität und dem prosozialen Verhalten zu den verschiedenen Messzeitpunkten untersucht (jeweils unter Kontrolle des Ausgangsniveaus und der weiteren Kontrollvariablen), wobei die Stichprobengröße zwischen 52 und 188 Schülerinnen und Schülern variierte. Während es bei den meisten Zeitpunkten keinen signifikanten Zusammenhang zwischen der wahrgenommenen Qualität von Angeboten zu sozialem Lernen und dem prosozialen Verhalten gab, ging eine höhere Qualität in Medienangeboten bei den meisten Messzeitpunkten mit einem stärker ausgeprägten prosozialen Verhalten einher. Wie bereits bei der 1. StEG-Förderphase fehlen aber auch hier Analysen, welche einen möglichen Einfluss der Angebotsqualität auf die nachfolgende Entwicklung von sozialen Kompetenzen untersuchen.

\section{Metaanalyse von Durlak et al. (2010) zu after-school Programmen}

In der bisher umfangreichsten Meta-Analyse zu after-school Programmen in den USA berücksichtigten Durlak et al. (2010) insgesamt 75 Studien, welche unter anderem die Wirkungen der Teilnahme an einem after-school Programm auf die Selbstwahrnehmung der Kinder (z.B. Selbstwert oder Selbstkonzept), auf die Verbundenheit mit der Schule (d.h. Gefühle und Einstellungen gegenüber der Schule), auf positive soziale Verhaltensweisen, auf problematische externalisierende Verhaltensweisen oder auf Drogengebrauch untersuchten. Je nach Konstrukt konnte dabei auf zwischen 23 und 43 Studien zurückgegriffen werden, wobei für jede Studie eine standardisierte mittlere Differenz zwischen der Interventionsgruppe (Nutzung des after- 
school Programmes) und der Kontrollgruppe (keine Angebotsnutzung), unter Berücksichtigung der Standardabweichung beider Gruppen, berechnet wurde.

In etwa zwei Drittel der Fälle erfolgte die Teilnahme am außerunterrichtlichen Angebot nicht zufällig. Ein Teil dieser nicht-randomisierten Studien griff auf bereits implementierte afterschool Programme zurück (z.B. Astroth \& Haynes, 2002; Baker \& Witt, 1996), wodurch auch Evaluationen mit einem ex-post-facto Design in die Meta-Analyse eingeflossen sind. Zudem fehlen bei den meisten Studien Angaben zum Ausgangsniveau der untersuchten sozialen Kompetenzen, oder es lag lediglich ein Querschnittdesign vor (z.B. Astroth \& Haynes, 2002), weshalb die meisten Analysen ohne Kontrolle allfälliger Unterschiede im Ausgangsniveau erfolgten - was angesichts der vielen quasi-experimentellen und ex-post-facto Designs nicht unproblematisch ist. Weitere Kontrollvariablen auf individueller Ebene oder auf Klassen- und Schulebene oder die Mehrebenenstruktur wurden ebenfalls nicht berücksichtigt. Kremer et al. (2015) kritisieren zudem, dass einige nicht-signifikante Effektstärken mit dem Wert Null geschätzt wurden, sofern diese aufgrund fehlender Angaben nicht berechnet werden konnten.

Die Analysen zur Teilnahme an after-school Programmen zeigten die erwünschten Effekte im Hinblick auf die Selbstwahrnehmungen, die Verbundenheit mit der Schule, positive soziale Verhaltensweisen, und externalisierendes Verhalten. Einzig beim Drogenkonsum zeigte sich kein generell signifikanter Effekt. Zusätzliche Analysen zeigten allerdings, dass diese Ergebnisse nur auf Programme zutreffen, die über spezifische Ziele mit entsprechenden Programmkomponenten verfügten und in denen aktive Lernformen angeboten wurden, welche miteinander verknüpft und koordiniert waren. Die Teilnahme an diesen sogenannten „SAFE“-Programmen (Sequences, Active, Focused, Explicit) hing zusätzlich mit einem niedrigeren Drogenkonsum zusammen. 


\section{Meta-Analyse von Kremer et al. (2015) zu after-school Programmen}

Eine weitere Meta-Analyse, welche unter anderem die Wirkungen einer Teilnahme an afterschool Programmen auf die Entwicklung von externalisierendem Verhalten untersuchte, wurde von Kremer et al. (2015) durchgeführt. Dabei wurden jedoch nur Studien berücksichtigt, bei denen mindestens die Hälfte der Stichprobe aus sogenannten „,children at-risk“ bestand, also aus Schülerinnen und Schülern mit einem erhöhten Risiko für schulischen Misserfolg. Zudem mussten ausreichend Informationen vorliegen, um eine Kontrolle hinsichtlich des Ausgangsniveaus im externalisierenden Verhalten zu ermöglichen. Aufgrund dieser Kriterien konnte eine Stichprobe von 24 Studien mit insgesamt 109‘282 Schülerinnen und Schülern, nahezu ausschließlich aus den USA, gewonnen werden. Externalisierendes Verhalten wurde dabei in 19 Studien untersucht. Wie bereits bei Durlak et al. (2010) wurde eine standardisierte mittlere Differenz zwischen der Interventionsgruppe (Nutzung des after-school Programmes) und der Kontrollgruppe (keine Angebotsnutzung, „treatment-as-usual“ oder ein alternatives Treatment) berechnet, wobei zusätzlich hinsichtlich des Ausgangsniveaus kontrolliert wurde.

Erneut wies der Großteil der Studien (70.8\%) ein quasi-experimentelles oder ex-post-facto Design auf (z.B. Pastchal-Temple, 2012), weshalb Kremer et al. (2015) bei mindestens 71 Prozent der Studien von einem hohen Risiko für Selektionseffekte ausgehen. Durch die Kontrolle des Ausgangsniveaus werden solche Selektionseffekte zum Teil kontrolliert, allerdings werden weitere mögliche Unterschiede, welche die Entwicklung des externalisierenden Verhaltens beeinflussen könnten, nicht berücksichtigt. Ebenso fehlt eine Kontrolle der Klassen- und Schulzugehörigkeit.

Die Analysen konnten keinen signifikanten Effekt der Angebotsnutzung auf die Entwicklung des externalisierenden Verhaltens identifizieren. Zwar zeigte sich ein hoher Grad an Heterogenität in den Ergebnissen der einzelnen Studien, der allerdings mittels der durchgeführten Moderatoranalysen nicht weiter erklärt werden konnte: Weder die Nutzungsintensität, die Art der 
Kontrollgruppe, das Alter der Schülerinnen und Schüler, das Design der Studien noch der Fokus der Programme beeinflussten die Ergebnisse in signifikantem Ausmaß.

\section{Meta-Analyse von Taheri und Welsh (2016) zu after-school Programmen}

Die jüngste Meta-Analyse zu after-school Programmen und deren Einfluss auf die Entwicklung von sozialen Kompetenzen stammt von Taheri und Welsh (2016), wobei Studien berücksichtigt wurden, die strafbare Handlungen oder antisoziales Verhalten als abhängige Variablen untersuchten. Dies führte zu einer Stichprobe von 12 Studien, größtenteils zu after-school Programmen aus den USA. Als Effektstärke wurde jeweils die standardisierte mittlere Differenz zwischen der Interventionsgruppe (Nutzung des after-school Programmes) und der Kontrollgruppe (keine Angebotsnutzung, ,treatment-as-usual“ oder ein alternatives Treatment) berechnet. Sofern Odds-Ratios oder partielle Korrelationen angegeben waren, wurden diese umgerechnet.

Auch bei Taheri und Welsh (2016) wiesen 75 Prozent der Studien ein quasi-experimentelles oder ein ex-post-facto Design auf (z.B. Mahoney, Stattin \& Magnusson, 2001; Posner \& Vandell, 1994), wobei offensichtlich nicht immer Angaben zum Ausgangsniveau vorliegen. Es bleibt allerdings unklar, bei wie vielen Studien dies der Fall ist und ob das Ausgangsniveau sofern vorhanden - in der Meta-Analyse berücksichtigt wurde. Dasselbe gilt für weitere Kontrollvariablen, die in einigen Originalstudien berücksichtigt wurden. Unberücksichtigt blieb auf jeden Fall eine allfällige Mehrebenenstruktur der einzelnen Studien.

Die Ergebnisse zeigten keinen signifikanten Einfluss der Teilnahme an after-school Programmen auf strafbare Handlungen oder antisoziales Verhalten, wobei keine getrennten Analysen für die beiden Konstrukte ausgewiesen wurden. Ähnlich wie bei Kremer et al. (2015) zeigte sich eine hohe Heterogenität, die allerdings nicht durch die berücksichtigten Moderatoren (u.a. Fokus der Programme, Alter der Schülerinnen und Schüler, Nutzungsdauer und Studiendesign) erklärt werden konnte. 


\section{Fazit zum Forschungsstand}

Im Hinblick auf die Studien zu Tages- und Ganztagsschulen kann zusammenfassend festgehalten werden, dass die Teilnahme an den verfügbaren Angeboten keinen Einfluss auf die Entwicklung des prosozialen Verhaltens zeigte (Fischer, Kuhn et al., 2011; Kanevski \& von Salisch, 2011; Sauerwein et al., 2018; Schüpbach et al., 2014). Etwas heterogenere Befunde sind für internalisierendes und insbesondere für externalisierendes Verhalten auszumachen (Fischer, Brümmer et al., 2011; Fischer, Kuhn et al., 2011; Kanevski \& von Salisch, 2011; Schüpbach et al., 2014), wobei körperlich aggressives Verhalten bei den Mädchen in der Stichprobe von Kanevski und von Salisch (2011) durch den Besuch von gebundenen Ganztagsschulen sogar verstärkt wurde. Als mögliche Moderatoren etablierten sich die Breite und Qualität der Angebote sowie die Beziehungen zum Betreuungspersonal, wobei allerdings ein Mangel an längsschnittlichen Befunden festzustellen ist. Analysen, welche spezifisch die Interaktionen mit dem Betreuungspersonal oder den Einfluss der Gleichaltrigen untersuchten, fehlen bislang.

Aufgrund der Stichprobengenerierung muss davon ausgegangen werden, dass die dargestellten Studien, welche ausnahmslos ein ex-post-facto Design aufweisen, von Selektionseffekten auf Ebene der Schulen und auf Ebene der Schülerinnen und Schüler betroffen sind. Einzig bei der Studie von Kanevski und von Salisch (2011) erfolgte eine grobe Parallelisierung auf Schulebene, was die Wahrscheinlichkeit von Selektionseffekten - zumindest in Bezug auf die berücksichtigten Merkmale - reduziert. Um mögliche Verzerrungen auf Ebene der Schülerinnen und Schüler zu kontrollieren, wurden in den verschiedenen Studien jeweils diverse Einflussfaktoren (z.B. Ausgangsniveau, Geschlecht, sozioökonomischer Status, Migrationshintergrund oder IQ) in den Analysen berücksichtigt. Im Gegensatz dazu beschränkten sich die kontrollierten Merkmale auf Schulebene, mit Ausnahme der bereits angesprochenen Studie von Kanevski 
und von Salisch (2011), höchstens auf die Schulform oder den Bindungsgrad, wobei die schulischen Merkmale pragmatisch auf Ebene der Schülerinnen und Schüler angesiedelt wurden (Fischer, Brümmer et al., 2011; Fischer, Kuhn et al., 2011; Sauerwein et al., 2018).

Die Mehrebenenstruktur der jeweiligen Stichproben und damit verbundene Unterschiede im Ausgangsniveau der einzelnen Klassen und Schulen, sowie in den entsprechenden Entwicklungsverläufen, wurden ebenfalls höchstens ansatzweise berücksichtigt: Zwar erfolgte bei einzelnen Studien eine Korrektur des Standardfehlers aufgrund der Schulzugehörigkeit (Fischer, Brümmer et al., 2011; Fischer, Kuhn et al., 2011; Sauerwein et al., 2018; Schüpbach et al., 2014), die Effektstärken wurden hingegen nicht angepasst. Gänzlich unberücksichtigt blieb die Klassenzugehörigkeit, wobei auch keine Angaben zur Varianz zwischen den Klassen gemacht wurden. Dies ist nicht unproblematisch, wie Van den Noortgate, Opdenakker und Onghena (2005) anhand von empirischen Bildungsdaten sowie einer Simulationsstudie zeigten: Insbesondere wenn die direkt angrenzenden Ebenen (im Falle der besprochenen Studien wäre dies die Klassenebene) unberücksichtigt bleiben, kann dies zu einer Verzerrung der Parameterschätzungen und der dazugehörigen Standardfehler führen. Dies soll kurz an einem Beispiel zur Entwicklung sozialer Kompetenz illustriert werden: So besteht die Möglichkeit, dass Klassen mit einem besonders vorteilhaften Entwicklungsverlauf hinsichtlich der sozialen Kompetenz überdurchschnittlich häufig an außerunterrichtlichen Bildungs- und Betreuungsangeboten teilnehmen, z.B. vermittelt durch ein positives Klassenklima und eine stärker ausgeprägte Schulmotivation, die sich gleichzeitig auf die Angebotsnutzung und auf die Entwicklung von sozialer Kompetenz auswirken. Die Nutzungshäufigkeit auf Klassenebene hätte demnach einen positiven Zusammenhang zur Entwicklung der sozialen Kompetenz, ohne dass die Angebotsnutzung durch die einzelnen Schülerinnen und Schüler automatisch einen förderlichen Effekt hat. Wird 
die Varianz zwischen den Klassen allerdings nicht kontrolliert, z.B. mittels Mehrebenenanalysen, könnte der Effekt einer Angebotsnutzung durch die einzelnen Schülerinnen und Schüler überschätzt werden.

Im Hinblick auf die Metaanalysen zu after-school Programmen zeigten sich einzig bei Durlak et al. (2010) positive Effekte, wobei durch den Besuch der after-school Programme prosoziales Verhalten gefördert wurde, während es bezüglich internalisierenden und externalisierenden Verhaltensweisen zu einem hemmenden Effekt kam. Im Gegensatz dazu konnten weder Kremer et al. (2015) noch Taheri und Welsh (2016) Effekte hinsichtlich der Entwicklung von externalisierendem Verhalten aufzeigen. Auffällig ist, dass bei allen drei Metaanalysen eine große Heterogenität in den Ergebnissen der Originalstudien vorliegt, wobei auch in einigen Studien unvorteilhafte Auswirkungen auf soziale Kompetenzen beobachtet wurden.

Die Vorgehensweise bei den beschriebenen Metaanalysen ist insbesondere auf randomisierte Studien ausgelegt, bei denen weder eine Kontrolle des Ausgangsniveaus noch von weiteren Kontrollvariablen notwendig ist. Dies steht allerdings im Widerspruch zu den jeweiligen Stichproben, die jeweils zu etwa zwei Dritteln aus Studien mit quasi-experimentellem oder ex-postfacto Design bestehen. Einzig bei Kremer et al. (2015) wurde konsequent eine Kontrolle des Ausgangsniveaus vorgenommen - weitere Kontrollvariablen blieben aber auch hier unberücksichtigt. Ebenso fehlt eine Berücksichtigung der Mehrebenenstruktur, die zumindest bei einigen der jeweils eingeflossenen Originalstudien vorlag. 


\section{Design und Fragestellungen}

Die Frage nach einer möglichen Wirkung von Tagesschulen auf die Entwicklung sozialer Kompetenzen wurde im Rahmen der vom Schweizerischen Nationalfonds finanzierten Längsschnittstudie EduCare-TaSe - Tagesschulen und Schulerfolg? untersucht. Die Grundgesamtheit der Studie umfasst sämtliche offenen Tagesschulen in der Deutschschweiz, die somit nebst dem Unterricht ein modular aufgebautes und freiwilliges außerunterrichtliches Bildungs- und Betreuungsangebot anbieten, das an mindestens drei Tagen die Woche jeweils am Mittag sowie am Nachmittag zur Verfügung steht. Aus forschungsökonomischen Gründen wurden bei den Schulen mindestens zwei parallele Erstjahrgangsklassen vorausgesetzt, wodurch kleine Schulen ausgeschlossen wurden. Von den insgesamt 251 angefragten und genannter Definition entsprechenden offenen Tagesschulen erklärten sich 53 Schulen aus 13 Deutschschweizer Kantonen bereit, an der Studie teilzunehmen, was zu einer Stichprobe von 1990 Schülerinnen und Schülern aus 120 Klassen führte.

Der Untersuchungszeitraum erstreckte sich von Ende der 1. (T1; Sommer 2014) bis Ende der 2. Jahrgangsstufe (T2; Sommer 2015). Dabei wurden Befragungen der Schul- und Tagesschulangebotsleitungen durchgeführt, um Informationen zum Ausbau des Tagesschulangebots, zu den Motiven hinter dem Ausbau und zur Angebotsnutzung durch die einzelnen Schülerinnen und Schüler zu gewinnen. Die sozialen Kompetenzen der Schülerinnen und Schüler wurden zu beiden Messzeitpunkten durch die Lehrpersonen eingeschätzt, wobei der „Strengths and Difficulties Questionnaire“(SDQ: Goodman, 1997, 2001) verwendet wurde. Die dabei gewonnenen Angaben lieferten einerseits Informationen zum internalisierenden, externalisierenden und prosozialen Verhalten der einzelnen Schülerinnen und Schülern. Zudem konnte für jede Klasse die durchschnittliche Ausprägung der jeweiligen Verhaltensweisen ermittelt werden, wobei nur die Schülerinnen und Schüler mit einer Nutzung des Tagesschulangebots berücksichtigt wurden. 
Die Interaktionen mit dem Betreuungspersonal wurden durch geschulte externe Beobachterinnen und Beobachter anhand der „Hort- und Ganztagsangebote-Skala“ (HUGS: Tietze, Roßbach, Stendel \& Wellner, 2007) bewertet, wozu jeweils ein halbtägiger Besuch erfolgte. Zusätzlich wurden Leistungstests bei den Schülerinnen und Schülern sowie Befragungen deren Eltern durchgeführt. Ein guter Überblick zu den verschiedenen Fragestellungen und Ergebnissen der EduCare-TaSe-Studie findet sich bei Schüpbach, Frei und Nieuwenboom (2018). Genauere Angaben zu der Stichprobengenerierung und den jeweils eingesetzten Messinstrumenten finden sich in den einzelnen Manuskripten.

Ausgehend vom theoretischen Hintergrund und dem bisherigen Forschungsstand wird in der vorliegenden Dissertation untersucht, ob die Entwicklung von externalisierendem, internalisierendem und prosozialem Verhalten durch die Nutzung der Tagesschulangebote beeinflusst wird. Dabei wird ein besonderes Augenmerk auf die Interaktionen im Tagesschulangebot gelegt, die zwar theoretisch eine zentrale Bedeutung haben, bisher aber kaum untersucht wurden. Einerseits wird davon ausgegangen, dass die Nutzung des Angebots insbesondere dann soziale Kompetenzen fördert, wenn die Interaktionen zwischen dem Betreuungspersonal und den Schülerinnen und Schülern von hoher Qualität sind. Andererseits dürften auch die Gleichaltrigen im Angebot einen Einfluss haben: Insbesondere, wenn die Gleichaltrigen kaum externalisierendes oder internalisierendes Verhalten zeigen, ist eine hemmende Wirkung auf das jeweilige Verhalten zu erwarten. Analog dazu dürften prosoziale Verhaltensweisen insbesondere dann gefördert werden, wenn diese bei möglichst vielen Gleichaltrigen im Angebot zu beobachten sind. Somit ergeben sich folgende Leitfragen:

- Fördert die Nutzung von Tagesschulangeboten soziale Kompetenzen, indem prosoziales Verhalten verstärkt und externalisierendes sowie internalisierendes Verhalten gehemmt wird? 
- Wird die Wirkung der Angebotsnutzung auf prosoziales, externalisierendes und internalisierendes Verhalten durch die Ausprägung der jeweiligen Verhaltensweisen bei den Gleichaltrigen im Tagesschulangebot moderiert?

- Wird die Wirkung der Angebotsnutzung auf prosoziales, externalisierendes und internalisierendes Verhalten durch die Interaktionsqualität im Tagesschulangebot, bezogen auf die Interaktionen zwischen dem Betreuungspersonal und den Schülerinnen und Schülern, moderiert?

Da die Nutzung der Tagesschulangebote freiwillig ist und sich auch die Nutzungshäufigkeit je nach Schule oder auch je nach Klasse unterscheidet, ist auch bei EduCare-TaSe von Selektionseffekten auf Schulebene, auf Klassenebene und auf individueller Ebene auszugehen. Um diese Selektionseffekte (und somit auch Kompositionseffekte) bestmöglich zu kontrollieren, werden zur Beantwortung der Fragestellungen Mehrebenenmodelle gerechnet. Dadurch wird berücksichtigt, dass sich das Ausgangsniveau und die Entwicklungsverläufe der sozialen Kompetenzen (1) zwischen den Schulen, (2) zwischen den Klassen innerhalb der Schulen, und (3) zwischen den Schülerinnen und Schülern innerhalb der Klassen unterscheiden. Merkmale auf Schul- und Klassenebene, welche die Entwicklungsverläufe beeinflussen könnten, werden damit effektiv kontrolliert, ebenso wie die Mehrebenenstruktur (d.h. die Klassen- und Schulzugehörigkeit) der Daten. Um mögliche Selektionseffekte auf individueller Ebene zu kontrollieren, werden Ausgangsniveau, kognitive Grundfähigkeiten und Geschlecht bei den Analysen berücksichtigt. 


\section{Manuskripte der Dissertation}

Die formulierten Fragestellungen dieser Dissertation wurden im Rahmen von vier Manuskripten untersucht, die im Folgenden kurz dargestellt werden.

Manuskript 1: Frei, L., Schüpbach, M., von Allmen, B. \& Nieuwenboom, W. (2016). Bildungsbezogene Erwartungen an Tagesschulen. Förderangebote an offenen Tagesschulen in der Deutschschweiz. Schweizerische Zeitschrift für Bildungswissenschaften, 38 (3), 549-567.

Die verbesserte Förderung sozialer Kompetenzen stellt nur eine von mehreren Erwartungen an Tagesschulen dar. Entsprechend können auch unterschiedliche Motive für den Ausbau der Tagesschulangebote verantwortlich sein und die Angebote können sich in ihrer Ausrichtung und Zielsetzung deutlich unterscheiden. In einem ersten Manuskript wurde daher anhand von 52 offenen Tagesschulen untersucht (1) welche Motive hinter dem Ausbau von Tagesschulen in der Deutschschweiz stehen, (2) wie stark die Schulleitung sowie Lehrpersonen an der konzeptionellen Ausarbeitung beteiligt waren und (3) welche individuellen und differenzierenden Förderelemente im Tagesschulgebot zur Verfügung stehen. Schließlich wurde auch geprüft, (4) ob die Bereitstellung solcher Förderelemente von Schulentwicklungsmerkmalen beeinflusst wird.

Ein bemerkenswerter Befund war, dass der Ausbau offener Tagesschulen in der Deutschschweiz primär eine Folge des sozialen Bedarfs in den jeweiligen Gemeinden darstellen dürfte. Eine mögliche Verbesserung der Bildungschancen oder der individuellen Förderung waren gemäß Angaben der Schulleitungen deutlich weniger relevant, ebenso der Wunsch der unterrichtenden Lehrpersonen. Dies widerspiegelt sich auch darin, dass die Lehrpersonen insgesamt kaum bis gar nicht an der konzeptionellen Ausarbeitung beteiligt waren. Bei etwa zwei Drittel der Tagesschulangebote beschränkten sich die Förderelemente auf Aufgabenbetreuung, teils ergänzt durch eine Aufgabenhilfe. Weitere Förderelemente, spezifisch für Schülerinnen und 
Schüler mit schwachen oder mit sehr guten Schulleistungen, sowie für fremdsprachige Schülerinnen und Schüler, standen lediglich bei etwa einem Drittel der Tagesschulangebote zur Verfügung. Dies war insbesondere dann der Fall, wenn vermehrt konzeptionelle Festlegungen zur Organisation der Tagesschulangebote gemacht wurden und wenn Lehrpersonen und Schulleitung stärker an der Ausarbeitung von Leitlinien beteiligt waren.

Manuskript 2: Frei, L., Schüpbach, M., Nieuwenboom, W. \& von Allmen, B. (2016). Extended Education and Externalizing Behavior. Utilization Intensity, Interaction Quality and Peers as Possible Moderators. International Journal for Research on Extended Education, 4 (2), 92109.

Im ersten Beitrag wurde deutlich, dass Tagesschulangebote in der Deutschschweiz anscheinend häufiger als Betreuungs- denn als Bildungsangebote verstanden werden. Dennoch stellen Tagesschulangebote soziale Settings dar, in denen soziale Kompetenzen gefördert werden können. In einem zweiten Manuskript wurde untersucht, welche Faktoren dabei eine Rolle spielen. Dazu wurde der Blick auf externalisierendes Verhalten gelegt, zu dem bereits einige - allerdings auch widersprüchliche - Befunde vorliegen (vgl. Kapitel 5: Forschungsstand). In diesem Beitrag wurde davon abgesehen, einen Vergleich zwischen den Schülerinnen und Schülern mit oder ohne Angebotsnutzung anzustellen. Stattdessen lag der Fokus auf den Nutzerinnen und Nutzern der Angebote und auf deren Nutzungsintensität, d.h. der Anzahl Stunden, die sie wöchentlich im Tagesschulangebot verbringen. Dabei wurde untersucht (1) ob die Nutzungsintensität die Entwicklung von externalisierendem Verhalten beeinflusst, (2) ob die Ausprägung von externalisierendem Verhalten bei den Gleichaltrigen im Tagesschulangebot die Entwicklung von externalisierendem Verhalten beeinflusst, (3) ob die Interaktionsqualität durch das Betreuungspersonal die Entwicklung von externalisierendem Verhalten beeinflusst und (4) ob die Nutzungsintensität einerseits mit dem externalisierenden Verhalten bei Gleichaltrigen und anderseits mit der Interaktionsqualität durch das Betreuungspersonal interagiert. 
Die Stichprobe für diese Analysen bestand aus 492 Schülerinnen und Schülern an 51 offenen Tagesschulen, wobei sämtliche Schülerinnen und Schüler das Tagesschulangebot während mindestens einem Jahr nutzten. Anhand von Mehrebenenmodellen wurden Wirkungen auf die Entwicklung von externalisierendem Verhalten von Ende der ersten bis Ende der zweiten Jahrgangsstufe untersucht, wobei eine leichte Abnahme von externalisierendem Verhalten zu beobachten war. Es zeigten sich keine Haupteffekte durch die Nutzungsintensität, das externalisierende Verhalten bei Gleichaltrigen oder die Interaktionsqualität durch das Betreuungspersonal. Allerdings gab es Interaktionseffekte mit der Nutzungsintensität: Je stärker die Ausprägung von externalisierendem Verhalten bei den Gleichaltrigen oder je niedriger die Interaktionsqualität durch das Betreuungspersonal, desto unvorteilhafter wirkte sich eine intensivere Nutzung der Angebote auf das externalisierende Verhalten aus.

Manuskript 3: Frei, L., Schüpbach, M., von Allmen, B. \& Nieuwenboom, W. (2021). Außerunterrichtliche Bildungs- und Betreuungsangebote als Mittel gegen externalisierendes Verhalten? Die Bedeutung der Gleichaltrigen und des Betreuungspersonals an Deutschschweizer Tagesschulen. Psychologie in Erziehung und Unterricht, 68 (2), 117-134.

Nachdem im zweiten Beitrag - im Hinblick auf die Teilstichprobe der Angebotsnutzerinnen und -nutzer - zumindest Hinweise auf unerwünschte Effekte durch externalisierendes Verhalten bei Gleichaltrigen und eine niedrige Interaktionsqualität durch das Betreuungspersonal gefunden wurden, wurde die Bedeutung der Gleichaltrigen und des Betreuungspersonals im dritten Beitrag weiter untersucht. Der zweite Beitrag lieferte zudem keine Informationen dazu, wie sich externalisierendes Verhalten bei den Schülerinnen und Schülern mit Angebotsnutzung im Vergleich zu den Schülerinnen und Schülern ohne Angebotsnutzung entwickelt. Im dritten Beitrag wurde deshalb die generelle Angebotsnutzung berücksichtigt, wobei Schülerinnen und Schüler mit einer dauerhaften Angebotsnutzung (über zwei Jahrgangsstufen) mit Schülerinnen und Schülern ohne Angebotsnutzung verglichen wurden. Die Fragestellungen sind ähnlich wie 
beim zweiten Beitrag: (1) Wird externalisierendes Verhalten durch die Nutzung des Tagesschulangebots verstärkt oder gehemmt? (2) Wird der Effekt der Angebotsnutzung auf das externalisierende Verhalten durch das externalisierende Verhalten der Gleichaltrigen im Tagesschulangebot moderiert? (3) Wird der Effekt der Angebotsnutzung auf das externalisierende Verhalten durch die Interaktionsqualität im Tagesschulangebot moderiert? (4) Beeinflusst die Interaktionsqualität, ob das externalisierende Verhalten der Gleichaltrigen einen moderierenden Effekt hat?

Um diese Fragen zu untersuchen, wurden Mehrebenenanalysen mit einer Stichprobe von 1596 Schülerinnen und Schülern aus 113 Klassen und 52 Schulen (unter Ausschluss der Schülerinnen und Schüler mit einer geringen Nutzungsdauer) durchgeführt. Es zeigte sich erneut eine leichte Abnahme von externalisierendem Verhalten zwischen Ende erste und Ende zweite Jahrgangsstufe, die allerdings durch eine Nutzung des Tagesschulangebots abgeschwächt wurde (vgl. Fragestellung 1). Dieser unerwünschte Effekt der Angebotsnutzung auf das externalisierende Verhalten zeigt sich insbesondere dann, wenn die Interaktionen mit dem Betreuungspersonal eine niedrige Qualität aufwiesen (vgl. Fragestellung 3). Das externalisierende Verhalten der Gleichaltrigen zeigte hingegen keinen allgemeinen moderierenden Effekt (vgl. Fragestellung 2), sondern nur in Tagesschulen mit einer unterdurchschnittlichen Interaktionsqualität (vgl. Fragestellung 4): Bei einer unterdurchschnittlichen Interaktionsqualität wirkte sich externalisierendes Verhalten der Gleichaltrigen unvorteilhaft auf die weitere Entwicklung des externalisierenden Verhaltens aus. Die Bedeutung der Gleichaltrigen und insbesondere des Betreuungspersonals konnte somit auf die Gesamtstichprobe und auf den Effekt einer generellen Angebotsnutzung übertragen werden.

Manuskript 4: Frei, L., Schüpbach, M., Nieuwenboom, W. \& Allmen, B. von (2018). Sozioemotionale Entwicklung. In M. Schüpbach, L. Frei \& W. Nieuwenboom (Hrsg.), Tagesschulen (S. 213-228). Wiesbaden: Springer Fachmedien Wiesbaden 
Nachdem die Mehrebenenanalysen im zweiten und dritten Beitrag unerwünschte Effekte einer Nutzung von Tagesschulangeboten auf die Entwicklung von externalisierendem Verhalten aufzeigten und die Bedeutung der Interaktionen im Tagesschulangebot verdeutlichten, wurden die Analysen aus dem dritten Beitrag auf die Entwicklung von internalisierendem sowie prosozialem Verhalten übertragen. Auch im Hinblick auf diese Verhaltensweisen liegen unterschiedliche Befunde zu außerunterrichtlichen Bildungs- und Betreuungsangeboten vor (vgl. Kapitel 5: Forschungsstand), wobei die bisherige Forschung aus Deutschland und aus der Schweiz bisher lediglich für internalisierendes Verhalten signifikante Wirkungen aufzeigte. Die Fragestellungen sind analog zum zweiten Beitrag: (1) Wird internalisierendes (respektive prosoziales) Verhalten durch die Nutzung des Tagesschulangebots verstärkt oder gehemmt? (2) Wird die Wirkung der Angebotsnutzung auf das internalisierende (respektive prosoziale) Verhalten durch die durchschnittliche Ausprägung von internalisierendem (respektive prosozialem) Verhalten bei den Gleichaltrigen im Tagesschulangebot moderiert? (3) Wird die Wirkung der Angebotsnutzung auf das internalisierende (respektive prosoziale) Verhalten durch die Interaktionsqualität im Tagesschulangebot moderiert?

Die Stichprobe für die Mehrebenenanalysen bestand aus 1076 Schülerinnen und Schülern aus 84 Klassen und 47 Schulen $^{2}$ und es wurden wiederum Schülerinnen und Schüler mit einer dauerhaften Nutzung (über zwei Jahrgangsstufen) und ohne Nutzung von Tagesschulangeboten verglichen. Während internalisierendes Verhalten von Ende erster bis Ende zweiter Jahrgangsstufe leicht abnahm, kam es beim prosozialen Verhalten zu einer Zunahme. Wie bereits beim externalisierenden Verhalten hatte die dauerhafte Angebotsnutzung insgesamt einen verstärkenden Effekt auf internalisierendes Verhalten, insbesondere, wenn die Gleichaltrigen im Tagesschulangebot vermehrt internalisierendes Verhalten zeigten. Die Interaktionsqualität durch

\footnotetext{
${ }^{2}$ Die Stichproben des 3. und 4. Artikels unterscheiden sich leicht, da der Umgang mit fehlenden Werten im 3. Artikel nachträglich angepasst wurde: Während im 4. Artikel Schülerinnen und Schüler mit fehlenden Werten ausgeschlossen wurden, wurden die fehlenden Werte im 3. Artikel mittels FIML («full information maximum likelihood») geschätzt.
} 
das Betreuungspersonal im Tagesschulangebot hatte hingegen keinen moderierenden Effekt. Hinsichtlich der Entwicklung von prosozialem Verhalten zeigte sich kein signifikanter Effekt durch die Angebotsnutzung, was auch nicht durch prosoziales Verhalten bei den Gleichaltrigen oder durch die Interaktionsqualität des Betreuungspersonals moderiert wurde. 


\section{Diskussion}

Diese Dissertation hat zum Ziel, den Einfluss einer Nutzung von Tagesschulangeboten auf die Entwicklung von sozialen Kompetenzen und spezifisch auf die Entwicklung von externalisierendem, internalisierendem sowie prosozialem Verhalten zu untersuchen. Darüber hinaus soll die Rolle der Interaktionen innerhalb des Tagesschulangebots näher beleuchtet werden: Wird die Wirkung von Tagesschulangeboten durch das Verhalten der Gleichaltrigen im Tagesschulangebot oder durch die Interaktionsqualität mit dem Betreuungspersonal moderiert? In einem ersten Diskussionsteil werden die Ergebnisse diskutiert und in den bisherigen Forschungsstand eingeordnet. Im zweiten Diskussionsteil werden Implikationen und Anregungen für die Praxis sowie für die weitere Forschung ausgeführt.

\section{Generelle Effekte der Nutzung von Tagesschulangeboten}

Hinsichtlich externalisierendem Verhalten wirkte sich eine dauerhafte Nutzung des Tagesschulangebots insgesamt leicht verstärkend auf externalisierendes Verhalten aus, was einem unerwünschten Effekt entspricht. Damit reiht sich der Befund in eine Reihe von Einzelstudien zu after-school Programmen ein, die ebenfalls verstärkende Effekte durch die Nutzung von auBerunterrichtlichen Bildungs- und Betreuungsangeboten auf externalisierende Verhaltensweisen feststellten (z.B. Mahoney et al., 2001; O’Hare, Biggart, Kerr \& Connolly, 2015; Wade, 2015). Auch für Ganztagsschulen zeigten sich bereits unerwünschte Teileffekte, wobei Kanevski und von Salisch (2011) eine verstärkende Wirkung auf das körperlich aggressive Verhalten von Mädchen fanden. Das Ergebnis steht jedoch im Widerspruch zur Mehrzahl der Studien, die entweder keine (vgl. Kremer et al., 2015; Schüpbach et al., 2014; Taheri \& Welsh, 2016) oder hemmende Effekte (Durlak et al., 2010; Fischer, Kuhn et al., 2011) von außerunterrichtlichen Bildungs- und Betreuungsangeboten auf externalisierendes Verhalten zeigten. 
Auch die Entwicklung des internalisierenden Verhaltens war von einem unerwünschten, leicht verstärkenden Effekt, durch die dauerhafte Nutzung des Tagesschulangebots betroffen. Damit unterscheidet sich das Ergebnis von früheren Studien aus dem deutschsprachigen Raum, die entweder keine oder erwünschte Effekte durch eine Angebotsteilnahme auf Teilaspekte des internalisierenden Verhaltens gefunden haben (Fischer, Brümmer et al., 2011; Kanevski \& von Salisch, 2011; Schüpbach et al., 2014) und auch vom Ergebnis der Metaanalyse von Durlak et al. (2010) zu after-school Programmen.

Der Befund, dass die Nutzung von Tagesschulangeboten keinen Einfluss auf die Entwicklung von prosozialem Verhalten hat, deckt sich zwar mit den bisherigen Ergebnissen aus dem deutschsprachigen Raum (Fischer, Kuhn et al., 2011; Kanevski \& von Salisch, 2011; Sauerwein et al., 2018; Schüpbach et al., 2014), widerspricht jedoch dem Befund von Durlak et al. (2010) zu after-school Programmen.

Die möglichen Gründe für die fehlenden oder gar unvorteilhaften Effekte einer Angebotsnutzung auf die Entwicklung in Teilbereichen der sozialen Kompetenz in der vorliegenden Untersuchung sind vielfältig. Grundsätzlich gilt festzuhalten, dass die außerunterrichtlichen Bildungs- und Betreuungsangebote sowohl zwischen den Ländern als auch innerhalb der einzelnen Länder starke Unterschiede aufweisen, sowohl was die Ziele, die Inhalte, die Nutzerinnen und Nutzer als auch die Qualität der Umsetzung angeht. Insgesamt deuten die Ergebnisse aus dem ersten Manuskript darauf hin, dass das Tagesschulangebot in der Deutschschweiz primär als Beitrag zur soziokulturellen Infrastruktur - als direkte Antwort auf die elterliche Nachfrage verstanden wird und somit die Betreuung im Vordergrund steht. Da keine Motive hinsichtlich einer verbesserten sozialen Integration oder darauf bezogene Angebotselemente erfragt wurden, sind diese natürlich nicht auszuschließen. Aber das «bedarfsgerechte Angebot» und der vorherrschende Betreuungscharakter deuten doch eher darauf hin, dass auch solche Motive und 
eine damit verbundene Ausrichtung auf eine konkrete sozio-emotionale Förderung im Tagesschulangebot eher eine Seltenheit darstellen dürften - zumindest in Bezug auf die verbreitete Form der offenen Tagesschulen.

Festzuhalten bleibt auch, dass die meisten Studien zu den Wirkungen von außerunterrichtlichen Bildungs- und Betreuungsangeboten von Selektionseffekten betroffen sind, was eine Generalisierung und eine Vergleichbarkeit der Ergebnisse erschwert. Dies trifft auch auf die vorliegende Untersuchung zu, da sich nicht sämtliche Schulen der Grundpopulation an der Studie beteiligten. Es ist zudem naheliegend, dass sich die Wirkungen auch zwischen den einzelnen Tagesschulen in unserer Stichprobe unterscheiden. Spannender ist demnach weniger die Frage, ob sich gewisse Effekte zeigen, sondern vielmehr, wieso sich diese Effekte einstellen. Im nächsten Abschnitt werden deshalb die Interaktionen im Tagesschulangebot näher beleuchtet.

\section{Moderierende Effekte durch das Verhalten der Gleichaltrigen}

Gemäß Radisch et al. (2008) stellen "akzeptierende und respektvolle Beziehungen zu anderen Kindern und Jugendlichen und zu Erwachsenen, die ein Gefühl der Zugehörigkeit geben, positive soziale Normen vermitteln und persönliches Wachstum unterstützen" (S. 278) eine von drei Basisdimensionen der Prozessqualität im außerunterrichtlichen Bildungs- und Betreuungsangebot dar. In der bisherigen Forschung zu Tages- und Ganztagsschulen wurden aber insbesondere die Beziehungen und Interaktionen zwischen den Schülerinnen und Schülern im Angebot kaum berücksichtigt. Die in der vorliegenden Dissertation präsentierten Befunde unterstützen nun erstmals diesen bereits theoretisch formulierten Moderationseffekt: Je mehr externalisierendes Verhalten von den Gleichaltrigen im Tagesschulangebot gezeigt wurde, desto unvorteilhafter wirkte sich eine intensivere Angebotsnutzung auf die Entwicklung von externalisierendem Verhalten aus (Manuskript 2). Auch im Vergleich zur Schülerschaft ohne Angebotsnutzung wirkte sich die dauerhafte Angebotsnutzung zunehmend verstärkend auf das externalisie- 
rende Verhalten aus, wenn die Gleichaltrigen im Angebot vermehrt entsprechende Verhaltensweisen zeigten (Manuskript 3) - dies jedoch begrenzt auf Tagesschulen mit einer unterdurchschnittlichen Interaktionsqualität durch das Betreuungspersonal. Auch hinsichtlich der Wirkungen einer dauerhaften Angebotsnutzung auf die Entwicklung von internalisierendem Verhalten wurde ein entsprechender moderierender Effekt durch das Verhalten der Gleichaltrigen festgestellt (Manuskript 4).

Wenn Mitschülerinnen und Mitschüler im Tagesschulangebot externalisierendes und internalisierendes Verhalten zeigen, scheint dies insbesondere bei einer intensiveren Nutzung des Angebots und bei einer niedrigeren Betreuungsqualität übernommen zu werden. In dieser Hinsicht zeigten sich also auch im Tagesschulangebot unerwünschte Lerneffekte, wie diese bereits in anderen Gruppensettings, inklusive dem regulären Schulunterricht, festgestellt wurden (vgl. Dishion \& Tipsord, 2011). Auffallend ist jedoch, dass die erwünschten Lerneffekte hinsichtlich prosozialem Verhalten ausblieben (Manuskript 4). Eine mögliche Erklärung besteht darin, dass externalisierendes und internalisierendes Verhalten zu Störungen führt und dadurch mehr Aufmerksamkeit generiert. Andererseits könnte es auch am eingesetzten Messinstrument liegen (Strengths and Difficulties Questionnaire: Goodman, 1997), welches insbesondere Schülerinnen und Schüler mit einem Defizit an prosozialem Verhalten, einem Überschuss an externalisierendem Verhalten oder einem Überschuss an internalisierendem Verhalten differenziert und bei den restlichen Schülerinnen und Schülern kaum zwischen unterschiedlichen Ausprägungen unterscheidet. Unterschiede zwischen Schülerinnen und Schülern mit einer mittleren und einer hohen Ausprägung von prosozialem Verhalten werden somit kaum erfasst. Lerneffekte sind aber insbesondere dann zu erwarten, wenn die anwesenden Mitschülerinnen und Mitschüler besonders viel prosoziales Verhalten zeigen. 


\section{Moderierende Effekte durch die Interaktionsqualität des Betreuungspersonals}

Die zuvor genannte Basisdimension von Radisch et al. (2008) bezieht sich aber nicht nur auf die Interaktionen mit den Gleichaltrigen sondern auch auf jene mit dem Betreuungspersonal. Eine bisherige Studie zu Ganztagsschulen fokussierte bereits die Beziehung zwischen der Schülerschaft und dem Betreuungspersonal, wobei eine von den Schülerinnen und Schülern als besser eingeschätzte Beziehung zum Betreuungspersonal mit weniger externalisierendem Verhalten und einem besseren Wohlbefinden zusammen hing (Fischer, Brümmer et al., 2011; Fischer, Kuhn et al., 2011). Befunde zu den konkreten Interaktionen im Angebot fehlten allerdings bislang für den deutschsprachigen Raum, weshalb die vorliegende Dissertation die mittels externer Beobachtung eingeschätzte Interaktionsqualität fokussiert: Wird der Effekt einer Tagesschulangebotsnutzung auf die Entwicklung von sozialen Kompetenzen durch die Interaktionsqualität des Betreuungspersonals moderiert? Ein solcher Effekt konnte einzig für externalisierendes Verhalten bestätigt werden: Je höher die Interaktionsqualität im Tagesschulangebot ausfiel, desto vorteilhafter wirkte sich eine intensivere Angebotsnutzung auf die Entwicklung von externalisierendem Verhalten aus (Manuskript 2) und dasselbe gilt für den Effekt der dauerhaften Angebotsnutzung im Vergleich zur Schülerschaft ohne Angebotsnutzung (Manuskript 3). Keine moderierende Wirkung zeigte sich hinsichtlich der Entwicklung von internalisierendem und prosozialem Verhalten (Manuskript 4).

Wieso zeigte sich der Moderationseffekt nur für externalisierendes aber nicht für internalisierendes und prosoziales Verhalten? Auch hier könnte die Operationalisierung eine Rolle spielen: Die eingesetzte Hort- und Ganztagsangebote-Skala (Tietze et al., 2007) erfasst unter anderem, wie es um die Beaufsichtigung steht, wie Verhaltensregeln und Disziplin umgesetzt werden, wie Begrüßung und Verabschiedung strukturiert sind und wie die Interaktionen zwischen den 
Schülerinnen und Schülern gestaltet werden. Möglicherweise wirken sich diese Merkmale stärker auf externalisierendes Verhalten aus, als dies bei internalisierendem und prosozialem Verhalten der Fall ist.

\section{Implikationen für die weitere Forschung}

Erstmals in der Forschung zu Tages- und Ganztagsschulen wurden Mehrebenenanalysen eingesetzt um den Einfluss der Angebotsnutzung auf die Entwicklung von sozialen Kompetenzen zu untersuchen. Dies ist besonders deshalb bemerkenswert, da die Ergebnisse im Vergleich zu ergänzenden Analysen, in denen die Schul- oder Klassenzugehörigkeit nur zur Kontrolle des Standardfehlers einbezogen wurde, zum Teil markante Unterschiede zeigten. Diese Beobachtung stärkt die bereits im Forschungsteil ausgeführte Empfehlung von Van den Noortgate et al. (2005), insbesondere die angrenzende Ebene (im vorliegenden Fall die Klassenebene, die an die im Fokus stehende Individualebene angrenzt) mittels Mehrebenenanalyse zu berücksichtigen.

Eine Neuheit stellt auch die Berücksichtigung der Gleichaltrigen im Tagesschulangebot dar. Bisherige Untersuchungen beschränkten sich hauptsächlich auf Merkmale des Angebots, des Betreuungspersonals, der einzelnen Schülerinnen und Schüler oder der wahrgenommenen Beziehungen der Schülerinnen und Schüler zum Betreuungspersonal. Die gefundenen Effekte hinsichtlich der Entwicklung von externalisierendem und internalisierendem Verhalten zeigen, dass die Bedeutung der Gleichaltrigen weiter untersucht werden sollte. Dabei dürften die Effekte in den vorliegenden Analysen sogar eher unterschätzt worden sein: Die Einschätzung des Verhaltens der Gleichaltrigen im Tagesschulangebot erfolgte eher indirekt, nämlich über eine Einschätzung durch die jeweilige Klassenlehrperson einer Schülerin oder eines Schülers, und berücksichtigte ausschließlich das im Unterricht gezeigte Verhalten derjenigen Klassenkolleginnen und -kollegen, welche das Tagesschulangebot nutzten. Eine Operationalisierung, die näher am tatsächlich beobachtbaren Verhalten bei den Gleichaltrigen im Tagesschulangebot 
dran ist (z.B. über eine Einschätzung des Betreuungspersonals, der Schülerinnen und Schüler oder durch eine standardisierte Beobachtung), könnte mehr Aufschluss über die tatsächliche Effektgröße geben. Zudem sollte untersucht werden, durch welche Maßnahmen unerwünschte Wirkungen der Gleichaltrigen abgeschwächt oder verhindert werden können. Ansatzpunkte bieten der Betreuungsschlüssel, die Qualifikationen des Betreuungspersonals, die Strukturierung und Beaufsichtigung des Angebots, aber auch die Akzeptanz des Angebots bei der Lehrerschaft, den Schülerinnen und Schülern sowie bei deren Eltern.

Eine Stärke der vorliegenden Untersuchung stellt die mittels standardisierter Beobachtung erhobene Interaktionsqualität dar. Die Hort- und Ganztagsangebote-Skala (Tietze et al., 2007) hat allerdings ein etwas spezielles System der Punktevergabe bei den einzelnen Items und ist insbesondere für die Tagesschulentwicklung geeignet: Es handelt sich nicht um eine LikertSkala, stattdessen müssen aufbauend einzelne Bedingungen erfüllt sein, um eine höhere Punktzahl zu erreichen. So müssen beispielsweise beim Item „Erzieher-Kind-Kommunikation“ die Bedingungen „Erzieherin initiiert kurze Gespräche, die nicht auf die Alltagsroutine bezogen sind“ und „Erzieherin geht auf einige Gesprächsinitiativen und Fragen der Kinder ein“ erfüllt sein, damit sich nachgeschaltete Merkmale zur Erzieher-Kind-Kommunikation auf die Punktevergabe bei diesem Item auswirken können. Künftige Studien sollten die Skala daher durch Fragebogen und weitere Beobachtungsinstrumente zur Interaktionsqualität ergänzen. Zudem erfolgte nur eine einmalige Beobachtung und nur durch eine Person, weshalb der Messfehler relativ groß sein dürfte. Um die Reliabilität der Einschätzung zu erhöhen, sollten mehrere Beobachtungen zu mehreren Zeitpunkten und durch mehrere Beobachter respektive Beobachterinnen erfolgen (Oh, Osgood \& Smith, 2015). Künftige Studien könnten auch untersuchen, ob die Interaktionsqualität durch weitere Merkmale (beispielsweise die Qualifikationen des Personals, den Betreuungsschlüssel oder gezielte Tagesschulentwicklungsmaßnahmen) beeinflusst wird, um entsprechende Schlüsse für die Praxis zu ziehen. 
Der Strengths and Difficulties Questionnaire (SDQ), welcher zur Erfassung von sozialen Kompetenzen eingesetzt wurde, ist ein gut etabliertes und ökonomisches Messinstrument, dessen Validität und Reliabilität in verschiedenen Studien bestätigt wurde (Goodman et al., 2010; Saile, 2007; Woerner, Becker \& Rothenberger, 2004). Allerdings dient das Messinstrument primär zur Identifikation von Kindern mit Defiziten in ihrer sozialen Kompetenz, die auch gegen aussen sichtbar werden - kleinere Veränderungen, insbesondere bei Kindern mit einer stärker ausgeprägten sozialen Kompetenz, werden hingegen weniger gut erfasst. Insofern sollten sich zukünftige Studien nicht ausschliesslich auf den SDQ verlassen sondern auch andere Messinstrumente berücksichtigen.

Eine weitere Einschränkung bei der vorliegenden Dissertation stellt der Untersuchungszeitraum dar. Um längerfristige oder verzögerte Effekte zu untersuchen, sollte eine Studie möglichst über mehrere Jahre angelegt sein. Für die vorliegende Untersuchung war eine Verlängerung der Erhebungsdauer zwar geplant, konnte aber mangels finanzieller Mittel nicht realisiert werden.

Eine Herausforderung in der Forschung zu Tages- und Ganztagsschulen stellt die Selbstselektion auf verschiedenen Ebenen dar: Selbst wenn eine Vollerhebung angestrebt wird, hängt die Stichprobe davon ab, ob sich Schulen, Lehrpersonen, Schülerinnen und Schüler sowie deren Eltern beteiligen. Wie bereits angesprochen, wurden Selektionseffekte auf Klassen- und Schulebene bei der vorliegenden Untersuchung mittels Mehrebenenanalysen kontrolliert, zudem wurden Ausgangsniveau, Geschlecht und kognitive Grundfähigkeiten auf Ebene der Schülerinnen und Schüler als Kontrollvariablen berücksichtigt. Eine andere Möglichkeit, Selektionseffekte zu kontrollieren, bietet Propensity Score Matching (Stuart, 2010). Dies erlaubt es, möglichst vergleichbare Interventions- und Kontrollgruppen zu generieren. Das Vorgehen ist insbesondere bei kleineren Stichproben zu empfehlen, die keine Mehrebenenanalysen zulassen, kann aber auch mit Mehrebenenanalysen kombiniert werden (Hong \& Raudenbush, 2016). 
Ein Aspekt, der in der vorliegenden Dissertation vernachlässigt wurde, ist die Perspektive der Schülerinnen und Schüler. Es ist unklar, wie die einzelnen Schülerinnen und Schüler die Tagesschulangebote nutzen, welche Motivation sie mitbringen oder wie sie sich emotional einbringen. Es ist auch nicht bekannt, wie die Schülerinnen und Schüler ihre Freizeit neben dem Tagesschulangebot verbringen und das gilt ebenso für die Schülerinnen und Schüler ohne Angebotsnutzung. Dies zu erheben stellt gerade kurz nach Schuleintritt eine Herausforderung dar, da die Schülerinnen und Schüler in diesem Alter nur begrenzt Auskunft geben können. Umso wichtiger erscheinen qualitative Untersuchungen, die den Alltag der Schülerinnen und Schüler und die Bedeutung des Tagesschulangebots abbilden können. Aber auch für quantitative Studien gilt es, neue Wege zu suchen, um diese Perspektive stärker zu berücksichtigen.

\section{Implikationen für die Praxis}

Wie im Manuskript 1 aufgezeigt wurde, bieten offene Tagesschulen in der Deutschschweiz primär ein «bedarfsgerechtes Angebot», wie dies auch in der interkantonalen Vereinbarung über die Harmonisierung der obligatorischen Schule (EDK, 2007) verlangt wird. Darüber hinaus bestehen keine nationalen Vorgaben und auch auf kantonaler Ebene bestehen große Unterschiede ob und wieweit Anforderungen an Tagesschulen gestellt werden (Schultheiss \& Stern, 2013). Insofern wäre es Aufgabe der Politik, zu klären, inwiefern mit Tagesschulen pädagogische Ziele verfolgt werden und daraufhin entsprechende Rahmenbedingungen zu schaffen. Dazu gehören Vorgaben zum Betreuungsschlüssel, zu den Qualifikationen des Personals, zu pädagogischen Konzepten, Maßnahmen zur Qualitätssicherung und natürlich die nötigen finanziellen Mittel. Dabei ist wichtig zu beachten, dass sich «pädagogische Ziele» nicht bloß an einer zusätzlichen Förderung orientieren, sondern auch daran, unerwünschte Effekte zu vermeiden. In diesem Zusammenhang gilt es auch, eine gute Durchmischung der Schülerschaft im Tagesschulangebot zu erreichen (Manuskripte 3 \& 4), wozu wiederum Angebote benötigt werden, die für alle Familien sowohl finanziell als auch inhaltlich attraktiv sind. 
Auf Schulebene gilt es, ein Bewusstsein für das (positive und negative) Potential von Tagesschulangeboten hinsichtlich der Entwicklung von sozialer Kompetenz zu schaffen und die nötigen Kompetenzen des Betreuungspersonals zu fördern. Die pädagogischen Ziele und die dazugehörigen Mittel sollten in pädagogischen Konzepten festgehalten und durch einen regelmäBigen Austausch sowie durch Weiterbildungen verankert werden. Wie im Manuskript 1 festgehalten, sollten die Konzepte möglichst unter Einbezug der Lehrpersonen und der Schulleitung erstellt werden. Um schädliche Effekte durch die Gleichaltrigen im Tagesschulangebot zu vermeiden, sollte auch auf Schulebene eine gute Durchmischung der Schülerschaft angestrebt werden. Damit ist gemeint, dass Tagesschulangebote nicht primär von Schülerinnen und Schülern mit Verhaltensauffälligkeiten genutzt werden sollten, sondern von einer breiten Schülerschaft. Ein Mittel dazu dürfte insbesondere ein attraktives Tagesschulangebot darstellen, aber auch eine intensive Kommunikation und Kooperation mit den Eltern, der Schulleitung und der Lehrerschaft. Schließlich könnten auch spezifische Interventionen im Tagesschulangebot und Kooperationen mit Fachpersonen dazu beitragen, soziale Kompetenzen zu fördern und allfällige Konflikte zu lösen. Als konkrete Maßnahmen um schädliche Wirkungen durch Gleichaltrige in sozialen Settings zu verringern schlagen Dishion und Tipsord (2011) eine ausreichend strukturierte (Aktivitäten, Verhaltensregeln, Disziplin) und beaufsichtigte Umgebung vor. 


\section{Literaturverzeichnis}

Aeberli, C. \& Binder, H.-M. (2005). Das Einmaleins der Tagesschule. Ein Leitfaden für Gemeinde- und Schulbehörden. Zürich: Avenir Suisse.

Astroth, K. A. \& Haynes, G. W. (2002). More than cows and cooking. Newest research shows the impact of 4-H. Journal of Extension, 40 (4), 1-10.

Baker, D. \& Witt, P. A. (1996). Evaluation of the impact of twoafter-school programs. Journal of Park and Recreation Administration, 14 (3), 60-81.

Bundesministerium für Bildung und Forschung. (2003). Ganztagsschulen. Zeit für mehr. Investitionsprogramm "Zukunft Bildung und Betreuung". Bonn: BMBF.

Coradi Vellacott, M., Hollenweger, J., Nicolet, M. \& Wolter, S. C. (2003). Soziale Integration und Leistungsförderung. Thematischer Bericht der Erhebung PISA 2000 (Bildungsmonitoring Schweiz). Neuchâtel: BFS.

Dishion, T. J. \& Tipsord, J. M. (2011). Peer Contagion in Child and Adolescent Social and Emotional Development. Annual Review of Psychology, 62 (1), 189-214.

Durlak, J. A., Weissberg, R. P. \& Pachan, M. (2010). A Meta-Analysis of After-School Programs That Seek to Promote Personal and Social Skills in Children and Adolescents. American journal of community psychology, 45 (3-4), 294-309.

EDK. (2007). Interkantonale Vereinbarung über die Harmonisierung der obligatorischen Schule (HarmoS-Konkordat). Zugriff am 22.07.2021. Verfügbar unter http://edudoc.ch/record/24711/files/HarmoS_d.pdf

EDK. (2015). Kantonsumfrage 2014/2015. Zugriff am 22.07.2021. Verfügbar unter https://edudoc.ch/record/122866/files/Archiv_KU_14_15_d.pdf

Fergusson, D. M., Horwood, L. J. \& Ridder, E. M. (2007). Conduct and attentional problems in childhood and adolescence and later substance use, abuse and dependence. Results of a 25-year longitudinal study. Drug and Alcohol Dependence, 88, S14-S26.

Fischer, N., Brümmer, F. \& Kuhn, H. P. (2011). Entwicklung von Wohlbefinden und motivationalen Orientierungen in der Ganztagsschule. Zusammenhänge mit der Prozess- und Beziehungsqualität in den Angeboten. In N. Fischer, H. G. Holtappels, E. Klieme, T. Rauschenbach, L. Stecher \& I. Züchner (Hrsg.), Ganztagsschule: Entwicklung, Qualität, Wirkungen. Längsschnittliche Befunde der Studie zur Entwicklung von Ganztagsschulen (StEG) (S. 227-245). Weinheim \& Basel: Beltz Juventa.

Fischer, N., Kuhn, H. P. \& Züchner, I. (2011). Entwicklung von Sozialverhalten in der Ganztagsschule. Wirkungen der Ganztagsteilnahme und der Angebotsqualität. In N. Fischer, H. G. Holtappels, E. Klieme, T. Rauschenbach, L. Stecher \& I. Züchner (Hrsg.), Ganztagsschule: Entwicklung, Qualität, Wirkungen. Längsschnittliche Befunde der Studie zur Entwicklung von Ganztagsschulen (StEG) (S. 246-266). Weinheim \& Basel: Beltz Juventa.

Frei, L., Schüpbach, M., Nieuwenboom, W. \& Allmen, B. von (2018). Sozio-emotionale Entwicklung. In M. Schüpbach, L. Frei \& W. Nieuwenboom (Hrsg.), Tagesschulen (S. 213228). Wiesbaden: Springer Fachmedien Wiesbaden. 
Frei, L., Schüpbach, M., Nieuwenboom, W. \& von Allmen, B. (2016). Extended Education and Externalizing Behavior. Utilization Intensity, Interaction Quality and Peers as Possible Moderators. International Journal for Research on Extended Education, 4 (2), 92-109.

Frei, L., Schüpbach, M., von Allmen, B. \& Nieuwenboom, W. (2016). Bildungsbezogene Erwartungen an Tagesschulen. Förderangebote an offenen Tagesschulen in der Deutschschweiz. Schweizerische Zeitschrift für Bildungswissenschaften, 38 (3), 549-567.

Frei, L., Schüpbach, M., von Allmen, B. \& Nieuwenboom, W. (2021). Außerunterrichtliche Bildungs- und Betreuungsangebote als Mittel gegen externalisierendes Verhalten? Die Bedeutung der Gleichaltrigen und des Betreuungspersonals an Deutschschweizer Tagesschulen. Psychologie in Erziehung und Unterricht, 68 (2), 117-134.

Fröhlich-Gildhoff, K. (2013). Verhaltensauffälligkeiten bei Kindern und Jugendlichen (2. Aufl.). Stuttgart: W. Kohlhammer.

Goodman, A., Lamping, D. L. \& Ploubidis, G. B. (2010). When to use broader internalising and externalising subscales instead of the hypothesised five subscales on the Strengths and Difficulties Questionnaire (SDQ): data from British parents, teachers and children. Journal of abnormal child psychology, 38 (8), 1179-1191.

Goodman, R. (1997). The Strengths and Difficulties Questionnaire. A Research Note. Journal of Child Psychology and Psychiatry, 38 (5), 581-586.

Goodman, R. (2001). Psychometric Properties of the Strengths and Difficulties Questionnaire. Journal of the American Academy of Child \& Adolescent Psychiatry, 40 (11), 1337-1345.

Hong, G. \& Raudenbush, S. W. (2016). Effects of Kindergarten Retention Policy on Children's Cognitive Growth in Reading and Mathematics. Educational Evaluation and Policy Analysis, 27 (3), 205-224.

Kanevski, R. \& von Salisch, M. (2011). Fördert die Ganztagsschule die Entwicklung sozialer und emotionaler Kompetenzen bei Jugendlichen? Zeitschrift für Erziehungswissenschaft, 14 (3), 237-259.

Kanning, U. P. (2002). Soziale Kompetenz - Definition, Strukturen und Prozesse. Zeitschrift für Psychologie, 210 (4), 154-163.

Klieme, E. (2006). Empirische Unterrichtsforschung: Aktuelle Entwicklungen, theoretische Grundlagen und fachspezifische Befunde. Zeitschrift für Pädagogik, 52 (6), 765-773.

Kremer, K. P., Maynard, B. R., Polanin, J. R., Vaughn, M. G. \& Sarteschi, C. M. (2015). Effects of After-School Programs with At-Risk Youth on Attendance and Externalizing Behaviors. A Systematic Review and Meta-Analysis. Journal of Youth and Adolescence, 44 (3), 616-636.

Kuhn, H. P., Fischer, N. \& Schoreit, E. (2016). Soziales Lernen von Jungen und Mädchen in der Ganztagsschule. Zur Bedeutung der Mitbestimmung in den Angeboten für die Entwicklung der schulbezogenen sozialen Verantwortungsübernahme. In N. Fischer, H. P. Kuhn \& C. Tillack (Hrsg.), Theorie, Forschung und Praxis zur Qualität von Ganztagsschulen (Theorie und Praxis der Schulpädagogik, Band 38, S. 148-167). Immenhausen: Prolog-Verlag.

Leadbeater, B. J., Thompson, K. \& Sukhawathanakul, P. (2016). Enhancing Social Responsibility and Prosocial Leadership to Prevent Aggression, Peer Victimization, and Emotional 
Problems in Elementary School Children. American journal of community psychology, 58 (3-4), 365-376.

Liu, J. (2004). Childhood Externalizing Behavior. Theory and Implications. Journal of Child and Adolescent Psychiatric Nursing, 17 (3), 93-103.

Mahoney, J. L., Stattin, H. \& Magnusson, D. (2001). Youth recreation centre participation and criminal offending. A 20-year longitudinal study of Swedish boys. International Journal of Behavioral Development, 25 (6), 509-520.

McLeod, J. D. \& Kaiser, K. (2004). Childhood Emotional and Behavioral Problems and Educational Attainment. American Sociological Review, 69 (5), 636-658.

Metsäpelto, R.-L., Pakarinen, E., Kiuru, N., Poikkeus, A.-M., Lerkkanen, M.-K. \& Nurmi, J.E. (2015). Developmental dynamics between children's externalizing problems, taskavoidant behavior, and academic performance in early school years. A 4-year follow-up. Journal of Educational Psychology, 107 (1), 246-257.

Miller, B. M. (2003). Critical hours. Afterschool programs and educational success. Quincy, MA: Nellie Mae Education Foundation.

Nelson, S. K., Layous, K., Cole, S. W. \& Lyubomirsky, S. (2016). Do unto others or treat yourself? The effects of prosocial and self-focused behavior on psychological flourishing. Emotion, 16 (6), 850-861.

O’Hare, L., Biggart, A., Kerr, K. \& Connolly, P. (2015). A Randomized Controlled Trial Evaluation of an After-School Prosocial Behavior Program in an Area of Socioeconomic Disadvantage. The Elementary School Journal, 116 (1), 1-29.

Oh, Y., Osgood, D. W. \& Smith, E. P. (2015). Measuring afterschool program quality using setting-level observational approaches. The Journal of early adolescence, 35 (5-6), 681713.

Pastchal-Temple, A. S. (2012). The effect of regular participation in an after-school program on student achievement, attendance, and behavior. Mississippi State: Mississippi State University.

Pierce, K. M., Bolt, D. M. \& Vandell, D. L. (2010). Specific Features of After-School Program Quality. Associations with Children's Functioning in Middle Childhood. American journal of community psychology, 45 (3-4), 381-393.

Posner, J. K. \& Vandell, D. L. (1994). Low-Income Children's After-School Care. Are There Beneficial Effects of After-School Programs? Child development, 65 (2), 440.

Radisch, F., Stecher, L., Fischer, N. \& Klieme, E. (2008). Was wissen wir über die Kompetenzentwicklung in Ganztagsschulen? In C. Rohlfs, M. Harring \& C. Palentien (Hrsg.), Kompetenz-Bildung. Soziale, emotionale und kommunikative Kompetenzen von Kindern und Jugendlichen (S. 275-288). Wiesbaden: VS Verlag für Sozialwissenschaften / GWV Fachverlage GmbH Wiesbaden.

Reijntjes, A., Kamphuis, J. H., Prinzie, P. \& Telch, M. J. (2010). Peer victimization and internalizing problems in children: a meta-analysis of longitudinal studies. Child abuse \& neglect, 34 (4), 244-252.

Rose-Krasnor, L. (1997). The Nature of Social Competence. A Theoretical Review. Social Development, 6 (1), 111-135. 
Rose-Krasnor, L. \& Denham, S. (2009). Social-emotional competence in early childhood. In K. H. Rubin, W. M. Bukowski \& B. Laursen (Hrsg.), Social, emotional, and personality development in context. Handbook of peer interactions, relationships, and groups (S. 162179). New York, NY, US: Guilford Press.

Roth, J. L., Malone, L. M. \& Brooks-Gunn, J. (2010). Does the amount of participation in afterschool programs relate to developmental outcomes? A review of the literature. American journal of community psychology, 45 (3-4), 310-324.

Saile, H. (2007). Psychometrische Befunde zur Lehrerversion des "Strengths and Difficulties Questionnaire“" (SDQ-L). Zeitschrift für Entwicklungspsychologie und Pädagogische Psychologie, 39 (1), 25-32.

Sauerwein, M., Lossen, K., Theis, D., Rollett, W. \& Fischer, N. (2018). Zur Bedeutung des Besuchs von Ganztagsschulangeboten für das prosoziale Verhalten von Schülerinnen und Schülern. Ergebnisse der Studie zur Entwicklung von Ganztagsschulen. In M. Schüpbach, L. Frei \& W. Nieuwenboom (Hrsg.), Tagesschulen (S. 269-288). Wiesbaden: Springer Fachmedien Wiesbaden.

Schultheiss, A. \& Stern, S. (2013). Familienergänzende Kinderbetreuung im Schulbereich. Stand in den Kantonen. Zürich: INFRAS im Auftrag der schweizerischen Konferenz der kantonalen Erziehungsdirektoren (EDK).

Schüpbach, M. (2010). Ganztägige Bildung und Betreuung im Primarschulalter. Qualität und Wirksamkeit verschiedener Schulformen im Vergleich. Wiesbaden: VS Verlag für Sozialwissenschaften.

Schüpbach, M. (2018). Was ist eine Tagesschule? Eine historische Herleitung der Zeitorganisation an Schulen und eine begriffliche Klärung. In M. Schüpbach, L. Frei \& W. Nieuwenboom (Hrsg.), Tagesschulen (S. 15-27). Wiesbaden: Springer Fachmedien Wiesbaden.

Schüpbach, M., Andrey, S. \& Arpagaus, A. (2018). Ziele und Konzeption der Tagesschule Tagesschulleitlinien im Fokus. In M. Schüpbach, L. Frei \& W. Nieuwenboom (Hrsg.), Tagesschulen (S. 119-144). Wiesbaden: Springer Fachmedien Wiesbaden.

Schüpbach, M., Frei, L. \& Nieuwenboom, W. (Hrsg.). (2018). Tagesschulen. Wiesbaden: Springer Fachmedien Wiesbaden.

Schüpbach, M., Ignaczewska, J. \& Herzog, W. (2014). Sozio-emotionale Entwicklung von Ganztagsschulkindern auf der Primarschulstufe. Zeitschrift für Entwicklungspsychologie und Pädagogische Psychologie, 46 (1), 11-23.

SKBF. (2007). Bildungsbericht Schweiz 2006 (2. Aufl.). Aarau: Schweizerische Koordinationsstelle für Bildungsforschung. Zugriff am 22.07.2021. Verfügbar unter http://www.skbfcsre.ch/fileadmin/files/pdf/bildungsberichte/2006/bildungsbericht_06_de.pdf

Slopen, N., Kubzansky, L. D. \& Koenen, K. C. (2013). Internalizing and externalizing behaviors predict elevated inflammatory markers in childhood. Psychoneuroendocrinology, 38 (12), 2854-2862.

Stecher, L., Radisch, F., Fischer, N. \& Klieme, E. (2007). Bildungsqualität außerunterrichtlicher Angebote in der Ganztagsschule. Zeitschrift für Soziologie der Erziehung und Sozialisation, 27 (4), 346-366. 
Stern, S., Iten, R., Schwab, S., Felfe, C., Lechner, M. \& Thiemann, P. (2013). Familienergänzende Kinderbetreuung und Gleichstellung. Zürich, St. Gallen: INFRAS, Schweizerisches Institut für Empirische Wirtschaftsforschung, Universität St. Gallen.

Stuart, E. A. (2010). Matching methods for causal inference: A review and a look forward. Statistical science : a review journal of the Institute of Mathematical Statistics, 25 (1), 121.

Taheri, S. A. \& Welsh, B. C. (2016). After-School Programs for Delinquency Prevention. A Systematic Review and Meta-Analysis. Youth Violence and Juvenile Justice, 14 (3), 272290.

Tietze, W., Roßbach, H.-G., Stendel, M. \& Wellner, B. (2007). Hort- und GanztagsangeboteSkala (HUGS). Feststellung und Unterstützung pädagogischer Qualität in Horten und außerunterrichtlichen Angeboten. Berlin: Cornelsen Scriptor.

Van den Noortgate, W., Opdenakker, M.-C. \& Onghena, P. (2005). The Effects of Ignoring a Level in Multilevel Analysis. School Effectiveness and School Improvement, 16 (3), 281303.

Wade, C. E. (2015). The longitudinal effects of after-school program experiences, quantity, and regulatable features on children's social-emotional development. Children and Youth Services Review, 48, 70-79.

Woerner, W., Becker, A. \& Rothenberger, A. (2004). Normative data and scale properties of the German parent SDQ. European Child \& Adolescent Psychiatry, 13 (S2), 3-10. 


\section{Manuskript 1}

Frei, L., Schüpbach, M., von Allmen, B. \& Nieuwenboom, W. (2016). Bildungsbezogene Erwartungen an Tagesschulen. Förderangebote an offenen Tagesschulen in der Deutschschweiz. Schweizerische Zeitschrift für Bildungswissenschaften, 38 (3), 549-567. https://doi.org/ $\underline{10.24452 / \text { sjer.38.3.4993 }}$ 


\section{Bildungsbezogene Erwartungen an Tagesschulen: Förderangebote an offenen Tagesschulen in der Deutschschweiz.}

\section{Lukas Frei, Marianne Schüpbach, Benjamin von Allmen und Wim Nieuwenboom}

Im aktuellen Bildungsdiskurs wird Tagesschulen gerade im Hinblick auf Bildungsgerechtigkeit und verbesserter individueller Förderung eine zentrale Rolle eingeräumt. Ob Deutschschweizer Tagesschulen die entsprechenden Lerngelegenheiten bieten, ist allerdings kaum bekannt. Dieser Beitrag diskutiert bildungsbezogene Erwartungen an Tagesschulen sowie gängige Qualitätsmodelle und untersucht anhand offener Tagesschulen aus der Deutschschweiz, welche Rolle pädagogische Motive bei der Gründung von Tagesschulen spielen, wie stark sich Schulleitung und Lehrpersonen an der konzeptionellen Ausarbeitung beteiligen, welche individualisierenden und differenzierenden Förderelemente im Tagesschulangebot verankert sind und ob Letztere von Schulentwicklungsmerkmalen beeinflusst werden.

\section{Einleitung}

In den letzten 15 Jahren wurde das Angebot an Tagesschulen - Schulen, welche neben dem regulären Unterricht zusätzliche Bildungs- und Betreuungsangebote während des ganzen Tages bieten - in der Deutschschweiz deutlich ausgebaut (Stern et al., 2013). Verbunden mit der zunehmenden Dichte an Tagesschulen und dem ebenfalls stark intensivierten Ausbau ganztägiger Bildungs- und Betreuungsangebote in Deutschland, kam es im deutschsprachigen Raum zu einer vertieften Auseinandersetzung mit der Qualität entsprechender Angebote und deren Funktionen innerhalb des Bildungssystems (Fischer et al., 2011; Schüpbach, 2010). Da Tagesschulen die Möglichkeit für erweiterte Lerngelegenheiten ausserhalb des Unterrichts bieten, beispielsweise durch stärker individualisierende und differenzierende Förderangebote, wecken sie Hoffnungen auf eine verbesserte individuelle Förderung und mehr Bildungsgerechtigkeit. Allerdings ist bisher kaum bekannt, ob entsprechende Lerngelegenheiten in Deutschschweizer Tagesschulen überhaupt angeboten werden und welche Entwicklungsprozesse hinter dem Ausbau von Tagesschulen stehen. Dieser Beitrag untersucht, welche Motive dem Ausbau offener Tagesschulen in der Deutsch- 
schweiz zugrunde liegen, wie stark Lehrpersonen und Schulleitungen an konzeptionellen Festlegungen zu den Tagesschulangeboten beteiligt sind und inwieweit Aufgabenhilfe und -betreuung sowie stärker differenzierende Förderangebote zur Verfügung stehen. Zudem wird untersucht, ob sich Entwicklungsmerkmale identifizieren lassen, welche den Ausbau solcher Förderangebote begünstigen.

\section{Erwartungen an Tagesschulen}

Die Schweizerische Konferenz der kantonalen Erziehungsdirektoren definiert Tagesschulen als «Schulen mit ganztägigen Betreuungsangeboten (inklusive Mittagsverpflegung) an mehreren Tagen pro Woche» (EDK, 2013, S. 349), was in etwa der Definition von Ganztagsschulen in Deutschland entspricht (Sekretariat der Ständigen Konferenz der Kultusminister der Länder in der Bundesrepublik Deutschland, 2011). Einige Tagesschulen werden als gebundene Tagesschulen geführt, wobei sämtliche Kinder neben den Unterrichtszeiten auch an bestimmten ausserunterrichtlichen Angeboten teilnehmen müssen. Häufiger trifft man aber auf offene Tagesschulen, d.h. Schulen, die den Kindern nebst dem Unterricht ein modulares Bildungs- und Betreuungsangebot anbieten, welches freiwillig genutzt werden kann. Diese ausserunterrichtlichen Angebote - im Folgenden als Tagesschulangebote bezeichnet - stehen in der Regel unter Aufsicht und Verantwortung der Schulleitung und werden von einer Tagesschulangebotsleitung geführt.

Gesellschaftliche Veränderungen im Verlauf des 20. Jahrhunderts, darunter die veränderten Sozialisationsbedingungen, der Wunsch nach einer besseren Vereinbarkeit von Familie und Beruf sowie ein Anstieg in der Bedeutung schulischer Bildung prägen im deutschsprachigen Raum den Diskurs um den Inhalt von Bildung und die Bedeutung des Bildungssystems (Schüpbach, 2010). Dabei werden gerade Tagesschulen oftmals als mögliche Antwort auf wachsende Herausforderungen und Ansprüche betrachtet, was sich auch in den vielfältigen Begründungen des Tagesschulausbaus zeigt (Holtappels \& Rollett, 2009): Erstens werden Tagesschulen als wertvoller Beitrag zur soziokulturellen Infrastruktur gesehen, welcher die Erwerbstätigkeit der Eltern erleichtert und auch den Kindern eine gesellschaftliche und bildungsbezogene Teilhabe ermöglicht. Zweitens sollen Tagesschulen dem zunehmenden Bedürfnis nach ausserfamilialer und institutionalisierter Sozialisation gerecht werden und zu sozialer Integration beitragen. Drittens erhofft man sich durch ausserunterrichtliche Aktivitäten eine veränderte und erweiterte Lernkultur mit differenzierten Lerngelegenheit, durch die alle Schülerinnen und Schüler erreicht und gefördert werden. Diese Erwartung hinsichtlich einer verbesserten schulischen Förderung bezieht sich insbesondere auf Schülerinnen und Schüler, die während und zum Schluss ihrer Bildungslaufbahn nur unzureichende schulische Leistungen vorweisen können. Von diesem Schulversagen sind Kinder aus Familien mit niedrigem sozioöko- 
nomischem Status und mit Migrationshintergrund überdurchschnittlich häufig betroffen, weshalb man sich durch die erweiterten Lerngelegenheiten im Tagesschulangebot viertens einen Abbau von herkunftsbedingter Chancenungleichheit im Bildungssystem erhofft.

\section{Tagesschulqual ität}

Der fortgeschrittene Ausbau von Tagesschulen und Ganztagsschulen sowie die damit verbundenen Erwartungen führten im letzten Jahrzehnt zu einer Intensivierung der wissenschaftlichen Auseinandersetzung mit deren Qualität. Während in Deutschland insbesondere die Studie zur Entwicklung von Ganztagsschulen [StEG] zu einer immensen Datensammlung und zahlreichen Veröffentlichungen führte (z.B. Fischer et al., 2011), wurden in der Deutschschweiz das vom Schweizerischen Nationalfonds finanzierte Forschungsprojekt EduCare - Qualität und Wirksamkeit der familialen und ausserfamilialen Bildung und Betreuung im Primarschulalter (Schüpbach, 2010) sowie dessen Nachfolgeprojekt EduCare-TaSe - Tagesschulen und Schulerfolg lanciert.

Wenn es darum geht, Qualitätskriterien für Tagesschulen oder Ganztagsschulen zu bestimmen, lassen sich zwei Perspektiven unterscheiden (Holtappels, 2009). Einerseits beschreibt Holtappels eine systematisch-normative Perspektive, die sich an sozialisations-, bildungs- und schultheoretischen Begründungen orientiert. Nebst normativen Bestimmungen, ab wann man überhaupt von einer Tagesschule spricht, geht es hier also primär darum, welche Funktionen und Aufgaben von Tagesschulen zu erfüllen sind. Im Gegensatz dazu bezieht sich die empirische Perspektive auf "theoriegeleitete und empirisch fundierte Erkenntnisse über Zusammenhänge von Qualitätsmerkmalen, Bedingungen und Wirkungen» (Holtappels, 2009, S. 11). Die beiden Perspektiven schliessen sich aber keineswegs aus, so bedürfen systematisch-normative Festlegungen einer «durch empirische Erkenntnisse fundierten Schultheorie» (Holtappels, 2009, S. 12) und auch empirisch abgestützte Qualitätsmodelle nehmen immer gewisse normative Haltungen ein, insbesondere in Bezug auf die angestrebten Wirkungen.

Übersichtsarbeiten zu gängigen Qualitätsmodellen von ausserunterrichtlichen Angeboten an Tagesschulen oder Ganztagsschulen offenbaren eine Vielfalt an Dimensionen und Strukturen, die sich zum Teil auch in den verwendeten Begrifflichkeiten und deren Bedeutung stark unterscheiden (z.B. Fischer et al., 2011; Holtappels, 2009; Schüpbach, 2010). Gemeinsam ist den meisten Modellen, dass im Sinne des CIPO-Modells (Context Input Process Output oder auch CIPP-Modell; Stufflebeam, 1972) zwischen einer Input- bzw. Kontextebene, einer Prozessebene und einer Output- bzw. Wirkungsebene unterschieden wird. Häufig wird auch dem Umstand Rechnung getragen, dass sich Qualitätsmerkmale auf das Bildungssystem, auf die Einzelschule, auf Klassen oder auf 
einzelne Kinder beziehen können. Ausgehend von Angebots-Nutzungs-Modellen (z.B. Helmke, 2003) berücksichtigen neuere Modelle zudem stärker, dass die Wirkung der Angebote auch von der Nutzung der einzelnen Schülerinnen und Schüler abhängt.

\section{Theoretisches Rahmenmodell zur Erklärung der Ausbauqualität}

Forschungsbemühungen zur Qualität von Tagesschulen lassen sich je nach Fragestellung stärker der Schuleffektivitätsforschung oder der Schulentwicklungsforschung zuordnen: Gemäss Fischer, Radisch, Theis und Züchner (2012) befasst sich die Schuleffektivitätsforschung mit den Bedingungen "guter Schulen» im Hinblick auf schulische Lernergebnisse, während die Schulentwicklungsforschung Schulqualität bezüglich deren Entwicklungsbedingungen untersucht. Ein Qualitätsmodell zu letzterem Forschungszweig unterscheidet drei Bedingungsfelder, welche die Ausbauqualität des Ganztagsbetriebs beeinflussen (Holtappels \& Rollett, 2008, 2009, Abs. 1). Da dieses Modell für die in diesem Artikel fokussierten Fragestellungen den stärksten Detaillierungsgrad aufweist, wird im Folgenden kurz auf die einzelnen Aspekte eingegangen.

Das theoretische «Rahmenmodell zur Erklärung der Ausbauqualität des Ganztagsbetriebes» (Holtappels \& Rollett, 2008, 2009) bildet den theoretischen Wirkungszusammenhang zwischen verschiedenen Schulentwicklungsmerkmalen und der Ausbauqualität der ausserunterrichtlichen Angebote einer Ganztagsschule ab, wobei es sich auf empirische Ergebnisse der Innovations- und Schulentwicklungsforschung abstützt (einen Überblick dazu bieten z.B. Rollett \& Holtappels, 2010). Unter "Ausbauqualität» wird dabei "das Ergebnis eines mehr oder minder gelungenen Ganztagsschulausbaus» (Holtappels \& Rollett, 2009, S. 22) verstanden, wobei hier ausgehend von bildungs- und schultheoretischen Begründungen des Ausbaus eine normative Wertung vorgenommen wird. Dazu gehören beispielsweise Umfang und Breite des Angebots, zeitlicher Umfang des Betriebes, Umfang der Schülerteilnahme am Angebot oder auch die Zufriedenheit der beteiligten Gruppen mit demselben. Als zentrale Bedingungsfelder der Ausbauqualität unterscheiden Holtappels und Rollett zwischen «Ziele und Konzeption», "Organisationskultur» sowie dem "Entwicklungsprozess»: Der Qualitätsbereich "Ziele und Konzeption» bezieht sich auf die Ziele, welche mit dem Angebot verfolgt werden, worunter auch die Gründungsmotive hinter dem Ausbau fallen, sowie den erreichten konzeptionellen Entwicklungsstand. Unter "Organisationskultur» werden insbesondere das Gelingen inner- und ausserschulischer Kooperation, die Innovationsbereitschaft und die Beteiligung verschiedener Personengruppen am Ganztagsbetrieb zusammengefasst. Der eigentliche «Entwicklungsprozess», welcher dem Ganztagsausbau zugrunde liegt, wird als eigener Qualitätsbereich angesehen, mit welchem unter anderem die Art 
und der Umfang von Entwicklungsmassnahmen sowie die Beteiligung interner und externer Gruppen oder Institutionen am Entwicklungsprozess gemeint sind. Im Hinblick auf die zuvor beschriebenen Qualitätsmodelle bleibt festzuhalten, dass mit dem Rahmenmodell von Holtappels und Rollett $(2008,2009)$ nur ein Ausschnitt der Tagesschulqualität abgebildet werden kann. So bleiben beispielsweise nationale oder kantonale Vorgaben, ausserschulische Akteure oder auch die Wirkungsebene unberücksichtigt.

\section{Forschungsstand}

Die genannten Erwartungen an eine erweiterte Lernkultur und mehr Bildungsgleichheit stellen klare Anforderungen an ein vielfältiges Förderangebot in Tagesschulen, in welchem individuelle Bedürfnisse der einzelnen Schülerinnen und Schüler berücksichtigt werden. Ob diese Anforderungen erfüllt werden, ist für die Deutschschweiz allerdings weitgehend unklar. Im Hinblick auf gesetzliche Regulierungen bestehen auf nationaler Ebene keinerlei pädagogische Vorgaben für Tagesschulen und auf kantonaler Ebene zeigen sich deutliche Unterschiede, ob und inwieweit solche Anforderungen existieren (Schultheiss \& Stern, 2013). Es ist denn auch nicht erstaunlich, wenn in Bezug auf Tagesschulen und deren Qualitätskriterien eine gewisse Konzeptlosigkeit konstatiert wird (Flitner, 2011). Im Folgenden wird ein kurzer Überblick über den bisherigen Forschungsstand zum Ausbau von Tagesschulen respektive von Ganztagsschulen gegeben. Für weitere Befunde aus der Innovations- und Schulentwicklungsforschung, welche auch dem theoretischen Rahmenmodell von Holtappels und Rollett (2008, 2009) zugrunde liegen, verweisen wir an dieser Stelle auf den Überblick bei Rollett \& Holtappels (2010).

Bisher fehlen für die Deutschschweiz Befunde zu den Motiven hinter dem Ausbau von Tagesschulen und zur Beteiligung unterschiedlicher Professionsgruppen an der Ausarbeitung von Konzepten. Eine bessere Datenlage präsentiert sich in Deutschland durch eine repräsentative Befragung im Rahmen von StEG (Holtappels, 2008): So zeigte sich, dass insbesondere die Verbesserung von Bildungschancen und der individuellen Förderung zentrale Motive für den Ausbau darstellten, während der soziale Bedarf im Stadtteil und insbesondere der Wunsch des Lehrerkollegiums weniger ausschlaggebend waren. Nach eigener Angabe der Lehrkräfte waren diese in 61\% der Fälle zumindest teilweise an der Erarbeitung des Ganztagsschulkonzepts beteiligt. Insgesamt war die Lehrerpartizipation an Entwicklungsarbeiten in Grundschulen deutlich höher als in Sekundarschulen und in offenen Ganztagsschulen niedriger als in gebundenen Ganztagsschulen.

Informationen zur Häufigkeit von individualisierenden und differenzierenden Fördermassnahmen an Deutschschweizer Tagesschulangeboten sind bisher ebenfalls kaum vorhanden. Schüpbach (2010) konnte aufzeigen, dass 
«Hausaufgabenhilfe und -betreuung» in Deutschschweizer Tagesschulen signifikant häufiger vorkommt als in anderen Schulformen. Dabei wurde allerdings auf eine Unterscheidung offener und gebundener Tagesschulen verzichtet und die einzelnen Förderangebote sind nicht weiter differenziert dargestellt. Für Deutschland zeigte die erste StEG-Erhebungswelle, dass im Jahr 2009 nahezu sämtliche Ganztagsschulen in Deutschland Hausaufgabenhilfe oder zumindest -betreuung angeboten haben. Etwas seltener wurden Förderunterricht für Schülerinnen und Schüler mit über- oder unterdurchschnittlich guten Fachleistungen (85\%) und spezifische Fördermassnahmen, z.B. für Schülerinnen und Schüler nichtdeutscher Muttersprache oder Herkunft (61\%) angeboten (Rollett, Lossen, Jarsinski, Lüpschen \& Holtappels, 2011). Auch für das Jahr 2012 zeigte die zweite StEG-Erhebungswelle, dass lernunterstützende Elemente in ausserunterrichtlichen Ganztagsangeboten in Deutschland weit verbreitet sind und dass dies auch für den Primarbereich gilt (Fischer, Klieme, Holtappels, Stecher \& Rauschenbach, 2013). Leider wurde bei diesen Ergebnissen nicht zwischen offenen und gebundenen Ganztagsschulen unterschieden, wobei gebundene Modelle einen grösseren Handlungsspielraum durch Schulleitung und Lehrpersonen bieten, z.B. hinsichtlich der Rhythmisierung von unterrichtlichen und ausserunterrichtlichen Elementen. Der hohe Anteil offener Ganztagsschulen (80\% im Jahr 2012) deutet immerhin darauf hin, dass Hausaufgabenbetreuung und weitere Förderangebote auch von der Mehrzahl offener Ganztagsschulen angeboten werden.

Erste empirische Analysen zur Überprüfung des Rahmenmodells zur "Ausbauqualität des Ganztagbetriebes» wurden im Rahmen des Forschungsprojekts StEG durchgeführt (Holtappels \& Rollett, 2008; Rollett \& Holtappels, 2010; Rollett et al., 2011). Spezifische Ergebnisse zu individualisierenden und differenzierenden Förderangeboten an Ganztagsschulen fehlen bisher, dafür wurden Breite und Umfang der Angebotsstruktur untersucht. Dabei zeigte sich für den Grundschulbereich, dass intensivere Entwicklungsbemühungen, eine stärkere Beteiligung der Lehrkräfte in den Ganztagsangeboten und eine flexiblere Zeitorganisation die Entwicklung von Angebotsbreite und -umfang positiv beeinflussten. Im Gegensatz dazu zeigte eine bessere Zusammenarbeit zwischen Lehrkräften und pädagogisch tätigem Personal einen leicht hemmenden Einfluss auf die Angebotsbreite, was mit einer möglicherweise stärker vorhandenen Fokussierung auf gewisse Angebotsmerkmale begründet wurde (Rollett et al., 2011). Für die Entwicklung der Angebotsbreite im Sekundarschulbereich zeigten stärker ausgeprägte pädagogische Entwicklungsziele und umfangreichere konzeptuellen Festlegungen einen positiven Effekt. Dasselbe gilt für die Innovationsbereitschaft und Zusammenarbeit in den Lehrerkollegien sowie eine stärkere Beteiligung der Lehrkräfte in den Ganztagsangeboten. Systematische Qualitätsentwicklung, externe Unterstützungsmassnahmen sowie eine intensivere Weiterentwicklung des Ganztagsschulkonzepts wirkten sich ebenfalls förderlich auf Angebotsbreite und -umfang aus (Rollett et al., 2011). 


\section{Fragestellungen}

Ausgehend vom bisherigen Forschungsstand lassen sich folgende Fragestellungen skizzieren, die im Weiteren untersucht werden:

(1) Welche Rolle spielten pädagogische Motive bei der Tagesschulgründung?

(2) Wie intensiv waren Schulleitung und Lehrpersonen an der Erarbeitung von Leitlinien zum Tagesschulangebot beteiligt?

(3) Wie stark sind Hausaufgabenhilfe und -betreuung sowie stärker differenzierende Förderangebote in den Tagesschulangeboten vertreten?

(4) Können Unterschiede in der Verfügbarkeit solcher Förderangebote durch pädagogische Motive, konzeptionelle Beteiligung der Schulleitung oder der Lehrpersonen und durch konzeptionelle Festlegungen zur Organisation der Tagesschulangebote erklärt werden?

\section{Me thode}

\section{Stichprobenziehung}

Die Daten für die nachfolgenden Analysen stammen vom laufenden Forschungsprojekt EduCare-TaSe - Tagesschulen und Schulerfolg?, welches durch den Schweizerischen Nationalfonds finanziert wird. Im Rahmen des Forschungsprojekts wurde eine Vollerhebung bei offenen Deutschschweizer Tagesschulen im Primarschulbereich durchgeführt, wobei aus forschungsökonomischen Gründen mindestens zwei parallele Erstjahrgangsklassen vorausgesetzt wurden. Ausgehend von der Definition der kantonalen Erziehungsdirektoren (EDK, 2013) wurde folgende Operationalisierung vorgenommen: Eine offene Tagesschule ist eine Schule, die ein modular aufgebautes und freiwilliges ausserunterrichtliches Bildungs- und Betreuungsangebot anbietet, welches an mindestens drei Tagen die Woche jeweils am Mittag sowie am Nachmittag zur Verfügung steht. Von den insgesamt 251 angefragten offenen Tagesschulen erklärten sich 53 bereit, an der Studie teilzunehmen, wodurch 13 Deutschschweizer Kantone vertreten sind. Da bei einer Tagesschule die Angaben zu den Förderangeboten fehlen, beschränkt sich dieser Artikel auf die verbleibenden 52 Tagesschulen.

\section{Datenerhebung}

Die Daten für den vorliegenden Artikel wurden zwischen Dezember 2013 und September 2014 bei den 52 Schul- und Tagesschulangebotsleitungen via online-Fragebogen erhoben. Dabei wurden Angaben zu verschiedenen Förderangeboten, zu pädagogischen Motiven hinter dem Tagesschulausbau, zu konzeptionellen Festlegungen in den Leitlinien der Tagesschulangebote und zur Beteiligung verschiedener Professionsgruppen an der konzeptionellen Arbeit erfragt. Dazu orientierten wir uns hauptsächlich an Messinstrumenten, welche bereits bei StEG eingesetzt wurden (Quellenberg, 2009), wobei wir die Fragen an den Schweizerischen Sprach- und Kulturraum adaptierten. 
Pädagogische Motive bei der Tagesschulgründung

Mittels Befragung der Schulleitungen wurde erhoben, inwieweit pädagogische Motive («Sozialer Bedarf in der Gemeinde», "Wunsch der unterrichtenden Lehrpersonen», "Verbesserung der Bildungschancen» und «Verbesserung der individuellen Förderung»; adaptiert von Quellenberg, 2009) zur Umwandlung der Schule in eine Tagesschule führten. Dazu mussten die vier Items auf einer vierstufigen Skala ( $0=$ "Trifft gar nicht zu», $1=$ "Trifft eher nicht zu", $2=$ «Trifft eher zu» und 3 = «Trifft voll und ganz zu») beurteilt werden. Die interne Konsistenz der Skala "pädagogische Motive» ist akzeptabel $(\alpha=0.74)$ und vergleichbar mit den Werten bei Quellenberg $(\alpha=0.78)$.

\section{Konzeptionelle Festlegungen zur Organisation}

Über die Tagesschulangebotsleitungen wurde das Ausmaß konzeptioneller Festlegungen zur Organisation der Tagesschulangebote erfasst (adaptiert von Quellenberg, 2009). Auf einer vierstufigen Skala ( $0=$ "Gar nicht», $1=$ "Ansatzweise», 2 = «Weitgehend» und $3=$ "Umfassend») wurde beurteilt, inwieweit in den Leitlinien der Tagesschulangebote Aussagen zu neun verschiedenen Arbeitsfeldern («Zeitorganisation und Rhythmisierung», «Schulräumliche Organisation und Gestaltung», «Elternmitarbeit bzw. Kooperation mit Eltern», etc.) gemacht wurden. Die Skala erzielte eine gute interne Konsistenz $(\alpha=0.86)$, was in etwa dem Wert bei Quellenberg entspricht $(\alpha=0.80)$.

Partizipation an (Weiter-) Entwicklung der Leitlinien Ebenfalls über die Tagesschulangebotsleitungen wurde für verschiedene Professionsgruppen erfragt, inwieweit diese an der (Weiter-) Entwicklung der Leitlinien zum Tagesschulangebot beteiligt waren $(0=$ "Gar nicht», $1=$ «In geringem Umfang», 2 = «In mässigem Umfang» und 3 = "In hohem Umfang»; adaptiert von Quellenberg, 2009). Da die Items nur eine geringe interne Konsistenz aufweisen $(\alpha=0.34)$ und sich auch nicht für eine Faktorenanalyse eignen $(\mathrm{KMO}=0.46$; Hutcheson \& Sofroniou, 1999), wurden keine Skalen gebildet. Stattdessen wurden für weitere Analysen die Einzelitems zur Partizipation durch die Schulleitung und zur Partizipation durch die Lehrpersonen verwendet, wobei letzteres aufgrund einzelner Ausreisser zusätzlich dichotomisiert $0=$ «keine Partizipation», $1=$ «Partizipation»; $\mathrm{M}=0.25, \mathrm{SD}=0.44$ wurde.

\section{Förderangebote}

Schliesslich wurde die Tagesschulangebotsleitung auch noch $\mathrm{zu}$ möglichen Förderangeboten im Tagesschulangebot befragt (adaptiert von Quellenberg, 2009). Dabei sollten die Tagesschulangebotsleiterinnen und -leiter auf einer sechsstufigen Skala $(0=$ «Nein», $1=$ "Ja, monatlich», $2=$ "Ja, mehrmals im Monat», $3=$ "Ja, wöchentlich», $4=$ "Ja, mehrmals die Woche» und $5=$ $=$ Ja, täglich») festhalten, ob und wie häufig verschiedene Förderangebote («Hausaufgabenhilfe», «Hausaufgabenbetreuung», «Förderung von Kindern mit schwachen 
Schulleistungen», "Förderung von Kindern mit sehr guten Schulleistungen» und «Förderung von Kindern mit nichtdeutscher Muttersprache») in den Tagesschulangeboten verfügbar waren.

Alter der Tagesschule

Um einen entsprechenden Einfluss zu kontrollieren, wurden die Schulleitungen zusätzlich danach befragt, seit wie vielen Jahren Tagesschulangebote geführt werden $(\mathrm{M}=7.90, \mathrm{SD}=7.89)$.

\section{Ergebnisse}

\section{Deskriptive Ergebnisse zu den Schulentwicklungs- merkmalen}

In Tabelle 1 sind die deskriptiven Ergebnisse zu den drei untersuchten Schulentwicklungsmerkmalen ersichtlich. Zusätzlich sind in Tabelle 2 die Antworthäufigkeiten zu den einzelnen pädagogischen Motiven und der konzeptionellen Partizipation dargestellt. Unter den erfragten pädagogischen Motiven für die Tagesschulgründung nahm der soziale Bedarf in der Gemeinde die zentralste Rolle ein: $82.7 \%(\mathrm{M}=2.23)$ der Schulleitungen stimmten zu, dass dieses Motiv zumindest teilweise für den Ausbau der Schule in eine Tagesschule verantwortlich war. Die Motive «Verbesserung der Bildungschancen» (48.1\%, $\mathrm{M}=1.35$ ), «Verbesserung der individuellen Förderung» $(25 \%, \mathrm{M}=1.02)$ und «Wunsch der unterrichtenden Lehrpersonen» $(25 \%, M=0.85)$ fanden hingegen deutlich weniger Zustimmung. Bezüglich der Ausarbeitung oder Weiterentwicklung von Leitlinien zum Tagesschulangebot wurde angegeben, dass die Schulleitung in 67.3\% der Fälle zumindest teilweise und zu 32.7\% in hohem Umfang beteiligt war. Im Gegensatz dazu beteiligten sich nur 25\% der Lehrpersonen zumindest teilweise und lediglich 5.8\% in hohem Umfang.

\section{Deskriptive Ergebnisse und Clusteranalyse zu}

Förderangeboten

Tabelle 3 zeigt die Verfügbarkeit der erfragten Förderangebote in den untersuchten Tagesschulen. Nahezu sämtliche Tagesschulen bieten täglich oder zumindest mehrmals die Woche Hausaufgabenbetreuung an. Konkrete Hausaufgabenhilfe steht hingegen noch in rund 50\% der Tagesschulen zur Verfügung und Förderangebote für bestimmte Schülerinnen und Schüler sind selten. Um mögliche Muster zu identifizieren, wurde mit den fünf Items eine hierarchische Clusteranalyse nach Ward durchgeführt. Eine Visualisierung des Anstiegs in der Fehlerquadratsumme mittels Dendrogramm und Struktogramm sprach deutlich für eine Lösung mit zwei Clustern. Mit den dadurch ermittelten Clusterzentren wurde im Anschluss eine nichthierarchische Clusteranalyse (k-means) durchgeführt, wodurch vier Fälle (7.7\%) das Cluster wechselten. Diese Lösung konnte 
durch eine Diskriminanzanalyse bestätigt werden, wobei 98.1\% der Fälle richtig klassifiziert wurden und schliesslich führte auch eine Kreuzvalidierung in 96.2\% der Fälle zu einer korrekten Zuordnung.

Aus Abbildung 2 ist ersichtlich, dass nahezu sämtliche Tagesschulangebote, unabhängig von der Gruppenzugehörigkeit, täglich oder mehrmals wöchentlich die Möglichkeit zur betreuten Bearbeitung von Hausaufgaben anbieten. Deutliche Unterschiede zeigen sich allerdings bezüglich der Verfügbarkeit von weiteren Förderangeboten: Hausaufgabenhilfe (z.B. durch die Lehrperson) steht im Cluster 1 nur in 37.1\% der Tagesschulangebote zur Verfügung und differenzierende Förderangebote für leistungsschwache, leistungsstarke oder fremdsprachige Schülerinnen und Schüler fehlen fast gänzlich (jeweils in höchstens $11.4 \%$ der Tagesschulangebote vorhanden). Im Gegensatz dazu bieten 88.2\% der Tagesschulangebote im Cluster 2 eine Hausaufgabenhilfe an und auch die differenzierenden Förderangebote sind jeweils in mindestens $70.6 \%$ der Tagesschulangebote anzutreffen. Während sich Tagesschulen im Cluster 1 also vorwiegend auf Hausaufgabenbetreuung beschränken, verfügen Tagesschulen im Cluster 2 über ein breiteres Förderangebot. Im nächsten Schritt soll anhand einer logistischen Regressionsanalyse untersucht werden, ob die Clusterzugehörigkeit mittels Schulentwicklungsmerkmalen vorhergesagt werden kann.

\section{Logistische Regressionsanalyse zur Vorhersage der Verfügbarkeit von Förderangeboten}

Um mögliche Wirkungen der beschriebenen Schulentwicklungsmerkmale auf die Verfügbarkeit von individualisierenden und differenzierenden Förderangeboten zu untersuchen, wurde schrittweise eine logistische Regressionsanalyse gerechnet, mit der Clusterzugehörigkeit $(0=$ "Hausaufgabenbetreuung»; $1=$ «Hausaufgabenbetreuung und weitere Förderangebote») als zu erklärende Variable (Tabelle 4). In einem ersten Schritt wurden die pädagogischen Motive in das Modell aufgenommen $\left(C h i^{2}=1.77, p=0.18\right)$, wobei diese keinen signifikanten Einfluss auf die Clusterzugehörigkeit zeigten $(p=0.20)$. Im zweiten Schritt wurde das Ausmass konzeptueller Festlegungen zur Organisation der Tagesschulangebote aufgenommen, wodurch ein signifikanter Anteil der Varianz erklärt werden konnte $\left(\Delta C h i^{2}=6.28, p<0.05\right)$ : Umfassendere konzeptuelle Festlegungen erhöhten die Wahrscheinlichkeit, nebst Hausaufgabenbetreuung auch noch weitere Förderangebote vorzufinden $(\operatorname{Exp}(b)=3.36, p<0.05)$. Im dritten Schritt wurde die Partizipation durch Schulleitung und Lehrpersonen an der Ausarbeitung der Leitlinien zum Tagesschulangebot in das Modell aufgenommen. Weder die Beteiligung der Schulleitung ( $p=0.14$ ) noch der Lehrpersonen $(p=0.21)$ zeigte einen signifikanten Einfluss auf die Clusterzugehörigkeit, gemeinsam leisteten die beiden Items aber einen signifikanten Beitrag zur erklärten Varianz $\left(\Delta C h i^{2}=8.05, \mathrm{p}<0.05\right)$. Das Ausmass konzeptueller Festlegungen zur Organisation der Tagesschulangebote zeigte nach wie vor einen signifikanten Einfluss auf die Clusterzugehörigkeit $(\operatorname{Exp}(b)=4.97, p<0.01)$. 
Insgesamt konnte dieses Modell 37\% der Varianz in der Verfügbarkeit von individualisierenden und differenzierenden Förderangeboten erklären. Im vierten und letzten Schritt wurde schliesslich das Alter der Tagesschule als Kontrollvariable hinzugefügt, welches allerdings keinen Einfluss auf die Clusterzugehörigkeit zeigte $(p=0.12)$ und das Modell nicht signifikant veränderte $(\Delta$ $\left.C h i^{2}=3.48, p=0.06\right)$.

\section{Diskussion}

Unsere Studie untersuchte anhand einer Stichprobe von offenen Tagesschulen, (1) welche Rolle pädagogische Motive bei der Tagesschulgründung spielten, (2) wie intensiv Schulleitung und Lehrpersonen an der Erarbeitung von Leitlinien zum Tagesschulangebot beteiligt waren, (3) wie stark Hausaufgabenhilfe und -betreuung sowie stärker differenzierende Förderangebote in den Tagesschulangeboten vertreten sind und (4) ob Unterschiede in der Verfügbarkeit solcher Förderangebote durch ausgewählte Schulentwicklungsmerkmale erklärt werden können.

\section{Pädagogische Motive \& konzeptionelle Beteiligung}

Die Ergebnisse zu den pädagogischen Motiven zeigen, dass der Ausbau offener Tagesschulen in der Deutschschweiz ganz im Gegensatz zu Deutschland (Holtappels, 2008) nur selten durch eine erhoffte Verbesserung der Bildungschancen oder der individuellen Förderung angeregt wurde und dass auch der Wunsch der unterrichtenden Lehrpersonen nur beim Ausbau weniger Tagesschulen eine Rolle spielte. Deutlich ausschlaggebender scheint hingegen der soziale Bedarf in der jeweiligen Gemeinde zu sein. Unsere Befunde zu den untersuchten Motiven verdichten demnach den Eindruck, dass bildungsbezogene Überlegungen für den gegenwärtigen Ausbau von Tagesschulen in der Deutschschweiz nur eine untergeordnete Rolle spielen und dass Tagesschulen primär eine Antwort auf die gewachsene Nachfrage nach ausserfamiliären Betreuungsformen durch Familien und durch die Wirtschaft darstellen. Vor diesem Hintergrund ist auch nicht weiter erstaunlich, dass Lehrpersonen nur bei 25\% der Tagesschulen zumindest teilweise an der Ausarbeitung von Leitlinien zum Tagesschulangebot beteiligt waren und dass selbst die Schulleitung zu einem Drittel überhaupt nicht involviert war.

\section{Individualisierende und differenzierende}

\section{Förderangebote}

Während fast alle untersuchten Tagesschulen Hausaufgabenbetreuung anbieten, sind konkrete Hausaufgabenhilfen und insbesondere Förderangebote für bestimmte Schülerinnen und Schüler, auch im Vergleich zu den Ganztagsschulen in Deutschland (z.B. Fischer et al., 2013), eher selten anzutreffen. 
Eine Clusteranalyse zeigte auf, dass sich etwa zwei Drittel der Tagesschulen fast ausschliesslich auf Hausaufgabenbetreuung beschränken, während das Angebot bei den restlichen Tagesschulen deutlich vielfältiger ausfällt. Insbesondere die differenzierenden Förderangebote für leistungsschwache, leistungsstarke oder fremdsprachige Schülerinnen und Schüler werden demnach selten alleine, sondern meist in Kombination mit weiteren Förderelementen angeboten.

\section{Einfluss von Schulentwicklungsmerkmalen auf die Verfügbarkeit von Förderangeboten}

Ausgehend vom «Rahmenmodell zur Erklärung der Ausbauqualität des Ganztagsbetriebes» (Holtappels \& Rollett, 2008) haben wir untersucht, ob ein vielfältigeres Förderangebot durch ausgewählte Schulentwicklungsmerkmale vorhergesagt werden kann. Dabei zeigte sich erstens, dass insbesondere Tagesschulangebote mit umfassenderen konzeptuellen Festlegungen über eine breitere Palette an Förderangeboten verfügen, was sich mit den Befunden von Rollett et al. (2011) zur Angebotsbreite an deutschen Ganztagsschulen im Sekundarschulbereich deckt. Dass eine konzeptuelle Verankerung der Gestaltungselemente, der Zeitorganisation oder der Verknüpfung von Unterricht und Tagesschulangebot zusätzliche Förderangebote anregen kann, ist naheliegend. Möglich wäre aber auch, dass die Förderangebote zu neuen konzeptuellen Festlegungen führen, z.B. wenn dadurch erstmals eine Kooperation zwischen Lehrpersonal und weiterem pädagogisch tätigem Personal stattfindet.

Zweitens zeigte die Beteiligung durch Lehrpersonen und Schulleitung bei der Ausarbeitung von Leitlinien zum Tagesschulangebot einen Einfluss auf die Verfügbarkeit von Förderangeboten. Für sich genommen wirkte sich allerdings weder die Partizipation der Lehrpersonen noch der Schulleitung auf die Verfügbarkeit der Förderangebote aus. Dieser Befund könnte ein Ergebnis unserer kleinen Stichprobe und der damit verbundenen geringen Teststärke sein, könnte aber auch auf ein komplexeres Zusammenspiel zwischen der Beteiligung der beiden Professionsgruppen hinweisen.

Drittens konnten wir keinen Einfluss der pädagogischen Motive hinter dem Tagesschulausbau auf die Verfügbarkeit von Förderangeboten feststellen. Dies ist insofern erstaunlich, als dass gerade die Motive «bessere Bildungschancen» und «bessere individuelle Förderung» einen konkreten Bezug zu den untersuchten Förderangeboten haben und bei StEG ein positiver Einfluss von stärker ausgeprägten pädagogischen Entwicklungszielen auf die Entwicklung der Angebotsbreite festgestellt wurde (Rollett et al., 2011). Allerdings scheinen diese beiden Motive bei nahezu sämtlichen Tagesschulen nur eine untergeordnete Rolle zu spielen, was den fehlenden Zusammenhang erklären könnte. Alternativ wäre es aber auch möglich, dass sich die Motive hinter der Tagesschulgründung nicht auf die konkreten Ziele und deren Umsetzung in den Tagesschulangeboten auswirken. 
Insbesondere die Analyse zum Einfluss der Schulentwicklungsmerkmale auf die Verfügbarkeit von Förderangeboten ist mit einigen Einschränkungen verbunden: So konnten wir (1) nicht sämtliche Bereiche abdecken, die gemäss Rahmenmodell von Holtappels und Rollett (2008) von Bedeutung sind, was Erkenntnisse über die genauen Wirkmechanismen erschwert. Ein Grund dafür liegt im bereits sehr umfangreichen Fragenkatalog, bei dem wir uns auf ausgewählte Aspekte von Schulentwicklungsmerkmalen beschränken mussten. Ein zweiter Grund stellt die Stichprobe dar, die bereits für die vorliegende Regressionsanalyse eher klein war und mit weiteren Items an ihre Grenze gestossen wäre. Als weitere Einschränkung ist denn auch (2) die kleine Stichprobe zu nennen. So war die Teststärke eher gering und die einzelnen Tagesschulen hatten einen starken Einfluss auf die gerechneten Modelle, was sich auch an teils auffälligen Residuen zeigte. Für die gefundenen Effekte spricht allerdings, dass sich diese auch unter Ausschluss von Ausreissern als einigermassen stabil erwiesen. Wie bereits angesprochen, erlaubt unser Beitrag (3) keine abschliessenden Aussagen zur Kausalität, da Förderangebote und Entwicklungsmerkmale im Querschnitt erhoben wurden und es sich bei den pädagogischen Motiven und der konzeptionellen Beteiligung nur um retrospektive Einschätzungen handelt - im Fall der konzeptionellen Beteiligung durch Lehrpersonen und Schulleitung gar um eine Fremdeinschätzung. (4) Schliesslich ist festzuhalten, dass unserer Analyse hinsichtlich der Verfügbarkeit von Förderangeboten nur einen Teilaspekt der Tagesschulqualität abdeckt und weitere Qualitätsmerkmale unberücksichtigt bleiben.

\section{Faz it}

Im gegenwärtigen Diskurs um Bildung nehmen Tagesschulen eine zentrale Rolle ein, wobei auch Hoffnungen hinsichtlich mehr Bildungsgerechtigkeit und einer verbesserten individuellen Förderung geäussert werden. Bezogen auf offene Tagesschulen aus der Deutschschweiz zeigen unsere Ergebnisse allerdings, dass bildungsbezogene pädagogische Motive beim Ausbau von Tagesschulangeboten nur selten von Bedeutung sind und dass sich gerade Lehrpersonen kaum an der konzeptionellen Ausarbeitung beteiligen. Zwar bieten nahezu sämtliche untersuchten Tagesschulangebote Hausaufgabenbetreuung an, darüber hinaus sind individualisierende und differenzierende Förderangebote allerdings nur bei etwa einem Drittel der Tagesschulen vorhanden. Ob solche Förderangebote zur Verfügung stehen und dadurch im Sinne aktueller Qualitätsmodellen eine Zunahme von Bildungserfolg und -gerechtigkeit erwartet werden kann, dürfte einerseits von konzeptionellen Festlegungen zur Organisation der Tagesschulangebote und andererseits von der Beteiligung durch Lehrpersonen und Schulleitung bei der Ausarbeitung von Leitlinien abhängen. 


\section{Literaturverzeichnis}

EDK (2013). Kantonsumfrage 2012/2013. Zugriff am 14.09.2015-unter http://edudoc.ch/ record/115194/files/2013.pdf

Fischer, N., Holtappels, H. G., Klieme, E., Rauschenbach, T., Stecher, L. \& Züchner, I. (2011). Ganztagsschule: Entwicklung, Qualität, Wirkungen. Längsschnittliche Befunde der Studie zur Entwicklung von Ganztagsschulen (StEG). Weinheim \& Basel: Beltz Juventa.

Fischer, N., Klieme, E., Holtappels, H. G., Stecher, L. \& Rauschenbach, T. (2013). Ganztagsschule 2012/2013. Deskriptive Befunde einer bundesweiten Befragung. Frankfurt: DIPF/ IFS/JLU/DJI.

Fischer, N., Radisch, F., Theis, D. \& Züchner, I. (2012). Qualität von Ganztagsschulen Bedingungen, Wirkungen und Empfehlungen. Expertise für die SPD Bundestagsfraktion. Frankfurt am Main.

Flitner, C. (2011). Absturzgefährdet - schulische Tagesbetreuung in der Schweiz. Zürich: vpod.

Helmke, A. (2003). Unterrichtsqualität erfassen, bewerten, verbessern. Seelze: Kallmeyer.

Holtappels, H. G. (2008). Ziele, Konzepte, Entwicklungsprozesse. In H. G. Holtappels (Hrsg.), Ganztagsschule in Deutschland. Ergebnisse der Ausgangserhebung der «Studie zur Entwicklung von Ganztagsschulen» (StEG) (2., korrigierte Aufl., S. 139-163). Weinheim: Juventa.

Holtappels, H. G. (2009). Qualitätsmodelle - Theorie und Konzeptionen. In I. Kamski, H. G. Holtappels \& T. Schnetzer (Hrsg.), Qualität von Ganztagsschule. Konzepte und Orientierungen für die Praxis (S. 11-25). Münster: Waxmann Verlag GmbH.

Holtappels, H. G. \& Rollett, W. (2008). Organisationskultur, Entwicklung und Ganztagsschulausbau. In H. G. Holtappels (Hrsg.), Ganztagsschule in Deutschland. Ergebnisse der Ausgangserhebung der «Studie zur Entwicklung von Ganztagsschulen» (StEG) (2., korrigierte Aufl., S. 209-226). Weinheim: Juventa.

Holtappels, H. G. \& Rollett, W. (2009). Schulentwicklung in Ganztagsschulen. Zur Bedeutung von Zielorientierungen und Konzeption für die Qualität des Bildungsangebots. In L. Stecher, C. Allemann-Ghionda, W. Helsper \& E. Klieme (Hrsg.), Ganztägige Bildung und Betreuung (Zeitschrift für Pädagogik, Beiheft, Bd. 54, S. 18-39). Weinheim u.a.: Beltz.

Hutcheson, G. \& Sofroniou, N. (1999). The multivariate social scientist. Introductory statistics using generalized linear models. London: Sage Publications.

Quellenberg, H. (2009). Studie zur Entwicklung von Ganztagsschulen (StEG) - ausgewählte Hintergrundvariablen, Skalen und Indices der ersten Erhebungswelle. Frankfurt am Main: Gesellschaft zur Förderung Pädagogischer Forschung [u.a.].

Rollett, W. \& Holtappels, H. G. (2010). Entwicklung von Ganztagsschulen in Deutschland. Analysen zum Ausbaustand der Ganztagsschullandschaft und zu Entwicklungsbedingungen der Schülerteilnahme. In N. Berkemeyer, W. Bos, H. G. Holtappels, N. McElvany \& R. Schulz-Zander (Hrsg.), Jahrbuch der Schulentwicklung. Daten, Beispiele und Perspektiven (S. 99-129). Weinheim: Beltz.

Rollett, W., Lossen, K., Jarsinski, S., Lüpschen, N. \& Holtappels, H. G. (2011). Ausserunterrichtliche Angebotsstruktur an Ganztagsschulen. Entwicklungstrends und Entwicklungsbedingungen. In N. Fischer, H. G. Holtappels, E. Klieme, T. Rauschenbach, L. Stecher \& I. Züchner (Hrsg.), Ganztagsschule: Entwicklung, Qualität, Wirkungen. Längsschnittliche Befunde der Studie zur Entwicklung von Ganztagsschulen (StEG) (S. 76-96). Weinheim \& Basel: Beltz Juventa.

Schultheiss, A. \& Stern, S. (2013). Familienergänzende Kinderbetreunng im Schulbereich. Stand in den Kantonen. Zürich: INFRAS im Auftrag der schweizerischen Konferenz der kantonalen Erziehungsdirektoren (EDK).

Schüpbach, M. (2010). Ganztägige Bildung und Betreuung im Primarschulalter. Qualität und Wirksamkeit verschiedener Schulformen im Vergleich. Wiesbaden: VS Verlag für Sozialwissenschaften. 
Sekretariat der Ständigen Konferenz der Kultusminister der Länder in der Bundesrepublik Deutschland (2011). Definitionenkatalog zur Schulstatistik. Zugriff am 14.09.2015-unter http://www.kmk.org/fileadmin/pdf/Statistik/Defkat2011.pdf

Stern, S., Iten, R., Schwab, S., Felfe, C., Lechner, M. \& Thiemann, P. (2013). Familienergänzende Kinderbetreuung und Gleichstellung. Zürich, St. Gallen: INFRAS, Schweizerisches Institut für Empirische Wirtschaftsforschung, Universität St. Gallen.

Stufflebeam, D. L. (1972). Evaluation als Entscheidungshilfe. In C. Wulf (Hrsg.), Evaluation. Beschreibung und Bewertung von Unterricht, Curricula und Schulversuchen (S. 113-145). München: Piper.

Schlagworte: Tagesschulen, Förderangebote, Bildungsgerechtigkeit, Tagesschulqualität, Schulentwicklung

\section{Enjeux éducatifs des écoles à horaire continu: quelques offres d'encouragement réalisées en Suisse alémanique}

\section{Résumé}

La thématique des horaires scolaires continus est au cœur des discussions actuelles sur l'éducation, notamment par rapport à la justice scolaire et au soutien individualisé. Toutefois, on manque encore d'informations sur les opportunités réelles d'apprentissage offertes par les écoles à horaire continu de Suisse alémanique. Cet article met en discussion les attentes éducatives envers ces écoles à horaire continu ainsi que les modèles de qualité en vigueur. Il examine également, pour les écoles à horaire continu de Suisse alémanique, les motivations pédagogiques qui prévalent à leur fondation, la participation des directions d'école et celle des enseignants lors de leur création, les différents dispositifs de soutien individualisé et différencié proposés tout en analysant si ceux-ci sont influencés par les développements actuels dans le champ scolaire.

Mots-clés: Écoles à horaire continu, offres d'encouragement, justice scolaire, qualité des écoles à horaire continu, développement scolaire 


\title{
Le sfide educative delle scuole a orario continuato: alcune proposte di promozione realizzate in Svizzera tedesca
}

Riassunto

L'orario scolastico continuato é una tematica importante nelle discussioni attuali in campo educativo, centrata soprattutto sull'equità scolastica e il sostegno individuale. Malgrado ciò, mancano ancora oggi le informazioni sulle reali opportunità d'apprendimento che esistono nelle scuole a orario continuato della Svizzera tedesca. L'articolo mette in discussione le aspettative scolastiche nei confronti delle scuole a orario continuato e i modelli di qualità di riferimento in vigore. L'articolo esamina inoltre le motivazioni pedagogiche che prevalgono nella fondazione di queste scuole, la partecipazione dei direttori e degli insegnanti durante la fondazione, $\mathrm{i}$ dispositivi di sostegno differenziato proposti e se questi sono influenzati dagli sviluppi attuali in campo educativo.

Parole chiave: Scuole a orario continuato, proposte di promozione, equità scolastica, qualità delle scuole a orario continuato, sviluppo scolastico.

\section{Educational expectations of all-day schools: Educational promotion at open-attendance all-day schools in Switzerland}

\begin{abstract}
Summary
In current debates on education, all-day schools are granted a central role especially in view of educational equity and improved individual educational support. However, whether all-day schools in the German-speaking part of Switzerland indeed offer learning opportunities in accordance with that is not known. This paper discusses educational expectations placed on all-day schools as well as current quality models and, looking at all-day schools in the Germanspeaking part of Switzerland, examines what role educational motives play in the establishing of all-day schools, to what extent school principals and teachers participate in conceptual development, what individualizing and differentiating elements of educational promotion are anchored within the offerings of all-day schools, and whether the offerings are influenced by features of school development.
\end{abstract}

Keywords: All-day schools, educational promotion, educational equity, all-day school quality, school development 
Abbi ldungsverzeichnis

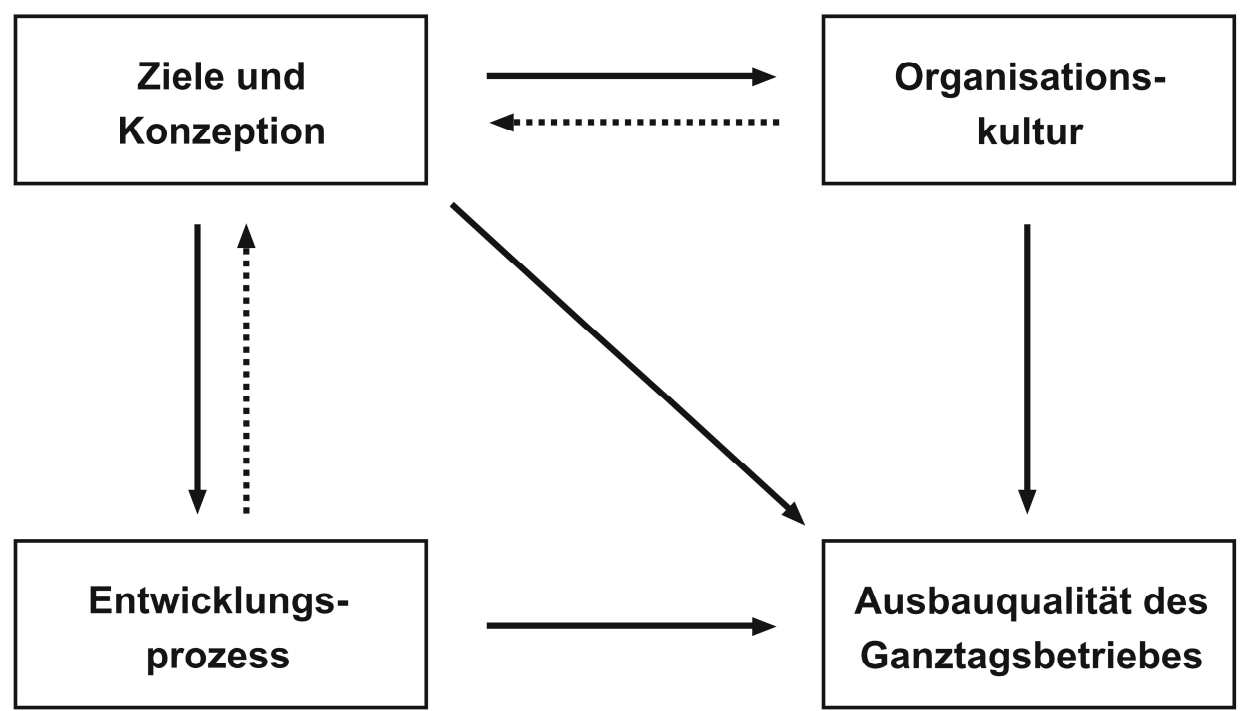

Abbildung 1. Theoretisches Rahmenmodell zur Erklärung der Ausbauqualität des Ganztagsbetriebes (Holtappels o Rollett, 2009, S. 23).



Abbildung 2. Verfügbarkeit von Förderangeboten in Abhängigkeit der Clusterzugehörigkeit. 
Tabel Lenverzeichnis

Tabelle 1. Deskriptive Ergebnisse zu den Schulentwicklungsmerkmalen.

\begin{tabular}{lcccc}
\hline & \# Items & $\begin{array}{c}\text { Skalie- } \\
\text { rung }\end{array}$ & M & SD \\
\hline Pädagogische Motive & 4 & $0-3$ & 1.36 & 0.65 \\
Konzeptionelle Festlegungen zur Organisation & 9 & $0-3$ & 1.44 & 0.67 \\
Partizipation durch Schulleitung & 1 & $0-3$ & 1.62 & 1.25 \\
Partizipation durch Lehrpersonen & 1 & $0-3$ & 0.38 & 0.80 \\
\hline
\end{tabular}

Anmerkungen: $N=52$

Tabelle 2. Antworthäufigkeiten zu den pädagogischen Motiven sowie zur konzeptionellen Partizipation.

\begin{tabular}{lcccc}
\hline Pädagogische Motive & $\begin{array}{c}\text { Trifft gar } \\
\text { nicht zu }\end{array}$ & $\begin{array}{c}\text { Trifft eher } \\
\text { nicht zu }\end{array}$ & $\begin{array}{c}\text { Trifft } \\
\text { eher zu }\end{array}$ & $\begin{array}{c}\text { Triff voll } \\
\text { und ganz } \\
\text { zu }\end{array}$ \\
\hline Sozialer Bedarf in der Gemeinde & $7.69 \%$ & $9.62 \%$ & $34.62 \%$ & $48.08 \%$ \\
Wunsch der unterrichtenden I ehrpersonen & $40.38 \%$ & $34.62 \%$ & $25.00 \%$ & $0.00 \%$ \\
Verbesserung der Bildungschancen & $23.08 \%$ & $28.85 \%$ & $38.46 \%$ & $9.62 \%$ \\
Verbesserung der individuellen Förderung & $26.92 \%$ & $48.08 \%$ & $21.15 \%$ & $3.85 \%$ \\
\hline Konzeptionelle Partizipation & Gar nicht & $\begin{array}{c}\text { In } \\
\text { geringem }\end{array}$ & $\begin{array}{c}\text { In } \\
\text { mässigem } \\
\text { Umfang }\end{array}$ & $\begin{array}{c}\text { In hohem } \\
\text { Umfang }\end{array}$ \\
\hline Partizipation durch Schulleitung & $32.69 \%$ & $5.77 \%$ & $28.85 \%$ & $32.69 \%$ \\
Partizipation durch Lehrpersonen & $75.00 \%$ & $17.31 \%$ & $1.92 \%$ & $5.77 \%$ \\
\hline
\end{tabular}

Anmerkungen: $N=52$

Tabelle 3. Antworthäufigkeiten hinsichtlich der Verfügbarkeit von Förderangeboten.

\begin{tabular}{lcccccc}
\hline & $\begin{array}{c}\text { Nicht } \\
\text { vorhanden }\end{array}$ & $\begin{array}{c}\text { Monat- } \\
\text { lich }\end{array}$ & $\begin{array}{c}\text { Mehrmals } \\
\text { im Monat }\end{array}$ & $\begin{array}{c}\text { Wöchent- } \\
\text { lich }\end{array}$ & $\begin{array}{c}\text { Mehrmals } \\
\text { die Woche }\end{array}$ & Täglich \\
\hline Hausaufgabenbetreuung & $3.85 \%$ & $0.00 \%$ & $0.00 \%$ & $0.00 \%$ & $17.31 \%$ & $78.85 \%$ \\
$\begin{array}{l}\text { Hausaufgabenhilfe } \\
\begin{array}{l}\text { Förderangebote für SuS mit } \\
\text { schwachen Schulleistungen }\end{array}\end{array}$ & $67.15 \%$ & $0.00 \%$ & $0.00 \%$ & $3.85 \%$ & $26.92 \%$ & $23.08 \%$ \\
$\begin{array}{l}\text { Förderangebote für SuS mit } \\
\text { sehr guten Schulleistungen }\end{array}$ & $75.00 \%$ & $1.92 \%$ & $0.00 \%$ & $3.85 \%$ & $17.31 \%$ & $9.62 \%$ \\
$\begin{array}{l}\text { Förderangebote für } \\
\text { fremdsprachige SuS }\end{array}$ & $59.62 \%$ & $1.92 \%$ & $1.92 \%$ & $7.69 \%$ & $11.54 \%$ & $17.31 \%$ \\
\hline
\end{tabular}

Anmerkungen: SuS=Schülerinnen und Schüler; $N=52$ 
Tabelle 4. Logistische Regression zur Vorhersage der Clusterzugehörigkeit ( $0=$ Aufgabenbetreunng / 1 =Aufgabenbetreuung und weitere Förderangebote).

\begin{tabular}{lcccc}
\hline Prädiktoren & $\begin{array}{c}\text { Modell 1 } \\
\operatorname{Exp}(\mathbf{b})\end{array}$ & $\begin{array}{c}\text { Modell 2 } \\
\operatorname{Exp}(\mathbf{b})\end{array}$ & $\begin{array}{c}\text { Modell 3 } \\
\operatorname{Exp}(\mathbf{b})\end{array}$ & $\begin{array}{c}\text { Modell 4 } \\
\operatorname{Exp}(\mathbf{b})\end{array}$ \\
\hline Pädagogische Motive & 1.88 & 1.90 & 1.78 & 1.24 \\
Konzeptionelle Festlegungen & & $3.36^{*}$ & $4.97^{* *}$ & $4.53^{*}$ \\
Partizipation durch LP & & & 3.73 & 2.76 \\
Partizipation durch SL & & & 1.59 & 1.60 \\
Alter der Tagesschule & & & & 1.19 \\
Konstante & $0.20^{*}$ & $0.03^{* *}$ & $0.01^{* *}$ & $0.00^{* *}$ \\
$\mathrm{R}^{2}$ (Nagelkerke) & 0.05 & 0.20 & 0.37 & 0.44 \\
Chi $^{2}$ & 1.77 & $8.05^{*}$ & $16.09^{* *}$ & $19.57^{* *}$ \\
$\Delta \mathrm{Chi}^{2}$ & 1.77 & $6.28^{*}$ & $8.05^{*}$ & 3.48 \\
\hline
\end{tabular}

Anmerkungen:LP=Lehrpersonen, $S L=$ Schulleitung; ${ }^{*} p \leq .05 ;{ }^{* *} p \leq .01 ; N=52$ 


\section{Manuskript 2}

Frei, L., Schüpbach, M., Nieuwenboom, W. \& von Allmen, B. (2016). Extended Education and Externalizing Behavior. Utilization Intensity, Interaction Quality and Peers as Possible Moderators. International Journal for Research on Extended Education, 4 (2), 92-109. https://doi.org/10.3224/ijree.v4i2.25783 


\title{
Extended Education and Externalizing Behavior: Utilization Intensity, Interaction Quality and Peers as Possible Moderators
}

\author{
Lukas Frei, Marianne Schuepbach, Wim Nieuwenboom and \\ Benjamin von Allmen
}

\begin{abstract}
So far, empirical evidence regarding the effects of extended education on externalizing behavior is mixed. To explore possible moderators, multilevel-analyses were conducted in a longitudinal sample of 492 students from 51 all-day schools in Switzerland. No main effects of utilization intensity, interaction quality and externalizing behavior in peers on the development of externalizing behavior from grade 1 to grade 2 were found. However, the relationship between utilization intensity and change in externalizing behavior was moderated by externalizing behavior in peers and by caregiver-student interactions. Subsequent analyses display a complex pattern of these cross-level interactions, indicating confounding characteristics. Implications for future research are discussed.
\end{abstract}

Keywords: extended education, all-day schools, externalizing behavior, quality, peer contagion

\section{Introduction}

Expectations regarding the benefit of extended education are manifold, including the promotion of prosocial and desirable behavior while counteracting externalizing behavior such as physical or verbal aggression, disruptive manners, delinquency and the like. And why should this not be the case? Extended education settings offer structure, supervision, activities and interactions with both peers and adults, which might otherwise be missing. However, empirical evidence so far is inconclusive, and some studies have even found adverse effects of extended education, amplifying the need to find out more about the conditions under which extended education succeeds in reducing externalizing behavior (Durlak, Weissberg, \& Pachan, 2010; Fischer, Kuhn, \& Züchner, 2011; Kremer, Maynard, Polanin, Vaughn, \& Sarteschi, 2015; O’Hare, Biggart, Kerr, \& Connolly, 2015; Schüpbach, Ignaczewska, \& Herzog, 2014; Wade, 2015). In this article, several possible moderators are addressed in a longitudinal sample of primary all-day schools in Switzerland: Does the degree of externalizing behavior in peers, the quality of interactions between caregivers and students, or the intensity of extended education utilization influence outcomes? 
Most primary schools in Switzerland provide school hours during five mornings a week and additional school hours in one to four afternoons (EDK, 2013). Beyond those regular school hours, supervision usually has to be organized by the parents. However, especially in the past decade, societal changes and political efforts have led to an increase in all-day schools across Switzerland (Stern et al., 2013), which are defined as schools not only comprising regular school hours but also offering education and care during the rest of the day (EDK, 2013). Those services (referred to as extended education) generally include lunch, a supervised program in the afternoon (e.g. activities, free-play, or homework), and, although less frequently, before-school care. While utilization of extended education is obligatory in some instances (obligatory all-day schools), most all-day schools implement an open-attendance model, referring to a modular system of extended education, which allows parents to decide whether their children use the respective services or not (voluntary all-day schools).

Since all-day schools offer additional opportunities to develop positive relationships in a structured and supervised environment, they are met with high societal and academic expectations regarding children's socio-emotional development (Aeberli \& Binder, 2005), including the reduction or prevention of externalizing behavior. Externalizing behavior refers "to a grouping of behavior problems that are manifested in children's outward behavior and reflect the child negatively acting on the external environment" (Liu, 2004, p. 93), such as disruptive, hyperactive and aggressive behavior. Externalizing behavior during the first school years has been found to reduce the probability of receiving a high school degree (McLeod \& Kaiser, 2004), was linked to low academic performance in higher grades (Metsäpelto et al., 2015), and predicted substance use, abuse and dependence in late adolescence and young adulthood (Fergusson, Horwood, \& Ridder, 2007). Additionally, even milder levels of externalizing behavior during school years seem to increase the likelihood of developing clinical disorders (Goodman, Lamping, \& Ploubidis, 2010).

\section{Review of the Literature}

\section{Effects of Extended Education on Externalizing Behavior}

So far, little is known about the actual effects of extended education in all-day schools on externalizing behavior, especially regarding such schools in Switzerland: In a sample of 295 first- to third-graders, Schüpbach et al. (2014) did not find a significant effect of participation in extended education on the development of parent-rated socio-emotional behavioral strengths (including items measuring hyperactivity). Additional research regarding all-day schools stems from Germany, where a comparable education and care system has been implemented. Fischer et al. (2011) examined 6'853 fifth-grade students, using 3 measurement points during 2005 to 2009. Participation in extended education was associated with a decrease in self-rated problematic behavior at school, as well as in self-rated violence and absenteeism. Kanevski and von Salisch (2011) explored physically aggressive behavior in a sample of 380 seventh-grade students. Male students from all-day schools showed a de- 
crease in peer-rated physically aggressive behavior during seventh-grade, compared to male students from half-day schools. However, for female students, the opposite results were found, favoring half-day schools.

Although not identical to all-day schools, after-school programs, which have been implemented in several other countries, share some properties in that they provide a structured and supervised setting for children after regular school hours, and offer a variety of social activities and academic enrichment. Yet, as the name implies, after-school programs are comprehensive programs, often targeting specific developmental aspects, and they are not part of the school itself (Kremer et al., 2015). Beside these differences and keeping in mind that all-day schools may also strongly vary in goals and activities, the extensive research on after-school programs provides some insight regarding the possible effects of extended education on externalizing behavior. However, results from meta-analyses are mixed (Durlak et al., 2010; Kremer et al., 2015). In fact, several studies even reported adverse effects of after-school programs on externalizing behavior (O'Hare et al., 2015; Wade, 2015), highlighting the need to consider and explore possible moderating aspects.

Since both after-school programs and all-day schools can be considered as prevention programs, the broader field of prevention research may offer valuable clues regarding such moderators. Extensive reviews of prevention efforts identified several features, distinguishing successful interventions from rather ineffective ones (Browne, Gafni, Roberts, Byrne, \& Majumdar, 2004; Nation et al., 2003), including sufficient dosage, opportunities to develop positive relationships, and well-trained personnel. An additional review points to potential negative influences of peers ("peer-contagion", Dishion \& Tipsord, 2011), especially when elevated levels of problematic behavior are prevalent.

\section{Utilization Intensity}

Among possible moderators, utilization intensity has probably received the biggest attention in past studies. In their review on participation in after-school programs, Roth, Malone, and Brooks-Gunn (2010) defined intensity as "frequency of attendance during one program year" (p. 314). Although some of the reviewed studies reported that intensity had a positive effect on various developmental outcomes, including problem behavior and peer relations, this was mainly the case when students with high participation were compared to students with no participation. Most studies exploring higher versus lower participation did not find that intensity was a significant influencing factor. The authors conclude that, "general statements proclaiming that greater participation in formal afterschool programs leads to improved outcomes are premature and inaccurate" (p. 321). As with general utilization, intensity alone does not seem to warrant positive effects which raises the question of possible moderators. Regarding the cited studies on all-day schools, only Schüpbach et al. (2014) explored a possible influence of intensity on externalizing behavior: Among 34 students utilizing extended education, utilization intensity was not linked to the development of socio-emotional behavioral strengths. 


\section{Caregiver-Student Interactions}

One of the main assumptions underlying expectations of positive socio-emotional outcomes through all-day schools and after-school programs is the provision of a structured environment where children experience positive interactions with peers and caregivers (Fischer et al., 2011; Wade, 2015). In line with those expectations, positive caregiver-student relations in after-school programs have been linked to a more favorable socio-emotional development, also with regard to externalizing behavior (e.g. Pierce, Bolt, \& Vandell, 2010; Wade, 2015). Even more importantly, in one study, participants who experienced negative relationships with their caregivers showed an increase in externalizing behavior compared to non-participants (Wade, 2015). With regard to all-day schools, Fischer et al. (2011) included caregiver-student relationship (rated by students) as predictor of problematic behavior at school, finding a negative association for all three time points. Unfortunately, they did not include caregiver-student relationships to predict change in problematic behavior nor as a possible moderator of the effect of all-day schools. Further, none of the other reviewed papers investigating all-day schools considered caregiver-student interactions in their analyses.

\section{Externalizing Behavior in Peers}

In their review regarding peer contagion, Dishion and Tipsord (2011) point out that peers can exert both positive and adverse influences during childhood and adolescence. The latter can be caused through deviancy training, which refers to interactions promoting deviant talk or behavior. For example, one reviewed paper studied coercive behavior in children and found that coercion by peers at age 5 had a significant effect on conduct problems at age 8 (Snyder et al., 2008). Such processes can be particularly problematic in settings, where children with elevated levels of externalizing behavior are aggregated, as is often the case in intervention studies but may also happen in general education settings, sometimes with adverse outcomes (Kellam, Ling, Merisca, Brown, \& Ialongo, 1998; Warren, Schoppelrey, Moberg, \& McDonald, 2005). Since many after-school programs specifically target students at risk, it may seem surprising that this aspect has received little to no attention so far. There are no publications we know of to date, that have explored the possible (moderating) effect of externalizing behavior in peers with regard to after-school programs or all-day schools.

\section{Hypotheses}

According to our review of the literature, previous studies produced mixed results regarding the effects of extended education on externalizing behavior. Therefore, it seems important to understand under which circumstances extended education can reduce externalizing behavior. While a possible moderating effect of utilization 
intensity has been studied in several articles, the quality of caregiver-student interactions and especially possible adverse effects of externalizing behavior in peers have received little to no attention. Taking a longitudinal approach towards change in externalizing behavior in a sample of first to second graders enrolled in extended education, this contribution aims to explore the following research questions: (1) Does the intensity of extended education utilization influence change in externalizing behavior? (2) Does the level of externalizing behavior in peers influence change in externalizing behavior? (3) Does the quality of caregiver-student interactions influence change in externalizing behavior? (4) With regard to change in externalizing behavior, does the intensity of extended education utilization interact with externalizing behavior in peers or with the quality of caregiver-student interactions? Consequently, the hypotheses with regard to main effects of possible moderators $(\mathrm{H} 1$ $-\mathrm{H} 3)$ and their interactions $(\mathrm{H} 4 \mathrm{a}-\mathrm{H} 4 \mathrm{~b})$ are formulated as following:

H1: Higher intensity of extended education utilization predicts change in externalizing behavior (i.e. increase or decrease) compared to lower intensity.

$\mathrm{H} 2$ : Higher levels of externalizing behavior in peers predict an increase in externalizing behavior compared to lower levels.

H3: Higher quality of caregiver-student interactions predicts a decrease in externalizing behavior compared to lower quality.

H4a: With increasing levels of externalizing behavior in peers, the effect of utilization intensity on externalizing behavior becomes more positive (i.e. less favorable: greater increase or smaller decrease in externalizing behavior as an effect of higher utilization intensity).

H4b: With an increasing quality of caregiver-student interactions, the effect of utilization intensity on externalizing behavior becomes less positive (i.e. more favorable: smaller increase or greater decrease in externalizing behavior as an effect of higher utilization intensity).

\section{Method}

\section{Sample}

Data for this article are drawn from the longitudinal research project EduCare-TaSe - All-Day School and School Success?, which is funded by the Swiss National Science Foundation. EduCare-TaSe is studying children in grades 1 and 2 at voluntary all-day schools, with some children utilizing extended education, whereas others do not. For economic reasons, only primary schools with at least two parallel classes at the primary school level were considered. Based on the definition proposed by the Swiss Conference of Cantonal Ministers of Education (EDK, 2013), all-day schools were defined as schools with (1) open-attendance, voluntary extended education, (2) extended education on at least 3 days per week, and (3) extended education at lunch- 
time and in the afternoon. Using estimates provided by the education departments of the cantons in German-speaking Switzerland, 251 primary all-day schools were identified, of which 53 schools with a total of 1'990 students agreed to participate, representing 13 cantons from the German-speaking part of Switzerland. By the end of grade 2, the sample decreased by 127 students, with additional students missing ratings regarding their participation in extended education (58 students), their externalizing behavior (234 students) or their sex (10 students). Only students enrolled in extended education for at least one year are considered for this article, resulting in a subsample of 492 students from 102 classes and 51 all-day schools which is used in subsequent analyses.

\section{Data-Collection}

Data-collection took place in between 2014 and 2015. At the end of grade 1 and again at the end of grade 2, class-teachers rated each of their students' externalizing behavior via online-survey. For each grade, intensity of extended education utilization was inquired through the head of extended education. Additionally, at the end of grade 1, quality of caregiver-student-interactions was rated via observational measure.

\section{Change in Externalizing Behavior}

The German version of the Strengths and Difficulties Questionnaire for teachers (SDQ; Goodman, 1997) was used to measure students' externalizing behavior. The SDQ for children and adolescents aged 4-17 consists of five subscales, including behavioral problems and hyperactivity, which can be rated by teachers, parents, or in the case of adolescents, by students themselves. Factorial structure, internal consistency, test-retest reliability, inter-rater agreement between parents and teachers, concurrent validity and predictive validity regarding subsequent clinical diagnoses has been explored in several international samples with generally good results, especially for the teacher version (Stone, Otten, Engels, Vermulst, \& Janssens, 2010). Regarding the German translation, factorial structure and internal consistency has been confirmed in a representative sample (Woerner, Becker, \& Rothenberger, 2004). However, because of the low discriminative validity between behavioral problems and hyperactivity in general population samples, Goodman et al. (2010) proposed a second-order factor to combine those two subscales into externalizing behavior. The resulting scale consists of ten items such as "Often fights with other children or bullies them", "Often has temper tantrums or hot tempers" or "Generally obedient, usually does what adults request" (the complete SDQ-survey including its German translation can be accessed on www.sdqinfo.org). Teachers are asked to rate each item with respect to a child's behavior over the last six months as "Not True" (0), "Somewhat True" (1) or "Certainly True" (2) and ratings are added up to a subscale representing externalizing behavior, with possible values from 0 to 20 . Internal consistency is good ( $\alpha=0.86$ for grade 1, 0.87 for grade 2 ) and comparable to those found by Goodman et al. $(2010 ; \alpha=0.88)$. Since the SDQ is a screening instrument 
with a non-normal distribution and a strong floor effect, the difference in externalizing behavior between the two time-points was calculated by subtracting the level of externalizing behavior at the end of grade 1 from the level of externalizing behavior at the end of grade 2 . The resulting dependent variable, representing change in externalizing behavior (scaled from -20 to 20 ), has an approximately normal distribution with positive scores indicating an increase and negative scores a decrease of externalizing behavior between $\mathrm{t} 1$ and $\mathrm{t} 2$, respectively $(\mathrm{M}=-0.48, \mathrm{SD}=2.60)$. To control for initial levels, externalizing behavior at the end of grade 1 was included as control variable $(\mathrm{M}=4.71, \mathrm{SD}=4.16)$.

\section{Student's Sex}

As a second control variable, student's sex was inquired through class lists and a short survey with students during grade 2 , and coded as male (0) or female (1), with $51 \%$ of the students being female.

\section{Utilization Intensity}

At the beginning of each grade, parents usually have to register their children for certain days and modules of extended education during that school year (e.g. each Wednesday from 11:45 am to 4:30 pm). For each child, intensity of extended education utilization (in minutes) during both grade 1 and grade 2 was inquired through the head of extended education. With regard to this paper, the average amount of hours spent taking part in extended education during both grades was calculated. On average students utilized extended education for 8.14 hours per week ( $\mathrm{SD}=7.24)$.

\section{Externalizing Behavior in Peers}

Since classmates constitute a student's primary peer group in school, we also expect them to spend more time together during extended education. Therefore, teachers' ratings of externalizing behavior in students utilizing extended education during grade 1 were aggregated at the class level, indicating higher or lower initial levels of externalizing behavior in peers, with possible values from 0 to $20(\mathrm{M}=4.65$ $\mathrm{SD}=2.69)$.

\section{Quality of Caregiver-Student Interactions}

At the end of grade 1, trained members of the research team took observations within extended education. Observations took place for at least four hours with the "Hortund Ganztagsangebote-Skala" (HUGS; Tietze, Rossbach, Stendel, \& Wellner, 2007), an adaption of the School-Age Care Environment Rating Scale (Harms, Jacobs, \& White, 1996) being used as rating instrument. HUGS consists of fifty features, of which nine features constitute a subscale measuring interactions. Since three of those features specifically tap into caregiver-parents interactions or aspects of collabora- 
tion, we only consider six features (i.e. communication between caregiver and children, interactions between caregiver and children, code of conduct / discipline, child supervision, welcome- and goodbye-procedures, handling of interactions between children) as representing caregiver-student interactions in this contribution. For each feature, ratings between 0 (inadequate quality) and 6 (excellent quality) were possible. Internal consistency for caregiver-student interactions is acceptable $(\alpha=0.72)$.

\section{Results}

\section{Analytic Strategy}

Multilevel regression analyses were performed using Mplus 7.3 (Muthén \& Muthén, 1998-2012). Descriptive statistics for all variables in our models are presented in table 1 . To test our hypotheses, several three-level analyses were performed to predict change in externalizing behavior, with students (level 1) nested into classes (level 2) nested into schools (level 3). First, we calculated a one-way ANOVA model (null-model) to determine the variation in the development of externalizing behavior for different levels. Second, we calculated several random-intercept models by adding predictors at the student level (model 1), the class level (model 2) and the school level (model 3).

Table 1. Descriptive Statistics.

\begin{tabular}{lccccc}
\hline & N & Min & Max & M & SD \\
\hline Students & & & & & \\
Change in Externalizing Behavior & 492 & -9.00 & 8.00 & -0.48 & 2.60 \\
Utilization Intensity & 492 & 0.50 & 36.67 & 8.14 & 7.24 \\
Externalizing Behavior (Grade 1) & 492 & 0.00 & 18.00 & 4.71 & 4.16 \\
Sex & 492 & 0.00 & 1.00 & 0.51 & 0.50 \\
Classes & & & & & \\
Externalizing Behavior in Peers & 102 & 0.00 & 13.50 & 4.65 & 2.69 \\
Schools & & & & & \\
Caregiver-Student Interactions & 51 & 1.20 & 6.00 & 4.48 & 0.95 \\
\hline
\end{tabular}

Measurement Scales: Change in Externalizing Behavior (-20 to 20), Utilization Intensity (ratio scale), Externalizing Behavior (0 to 20), Sex ( $0=$ male, $1=$ female), Externalizing Behavior in Peers (0 to 20), Caregiver-Student Interactions (0 to 6) 
Finally, we calculated an intercept-and-slopes-as-outcomes model (model 4; figure 1) to explore possible cross-level interactions. As an estimator, we used ML estimation with robust standard errors (MLR) and predictors were centered at the grand-mean. Additionally, externalizing behavior in peers and caregiverstudent interactions were z-standardized. Unstandardized coefficients are reported and we calculated $\mathrm{R}^{2}$ for each level by comparing the initial variance estimates to those in the respective models as proposed by Heck and Thomas (2015). Since the total amount of variance on each level also varies across models, $\mathrm{R}^{2}$-estimates have to be considered with caution. Therefore, we further provide log-likelihood and AIC to allow for a better comparison of different models, with lower values indicating better model-fit (Byrne, 2012). A robust chi-square difference test based on log-likelihood and scaling correction factors was used to compare models (http://www.statmodel.com/chidiff.shtml), as proposed by Muthén and Muthén (1998-2012).

Figure 1. Intercept-and-Slopes-as-Outcomes (Model 4).

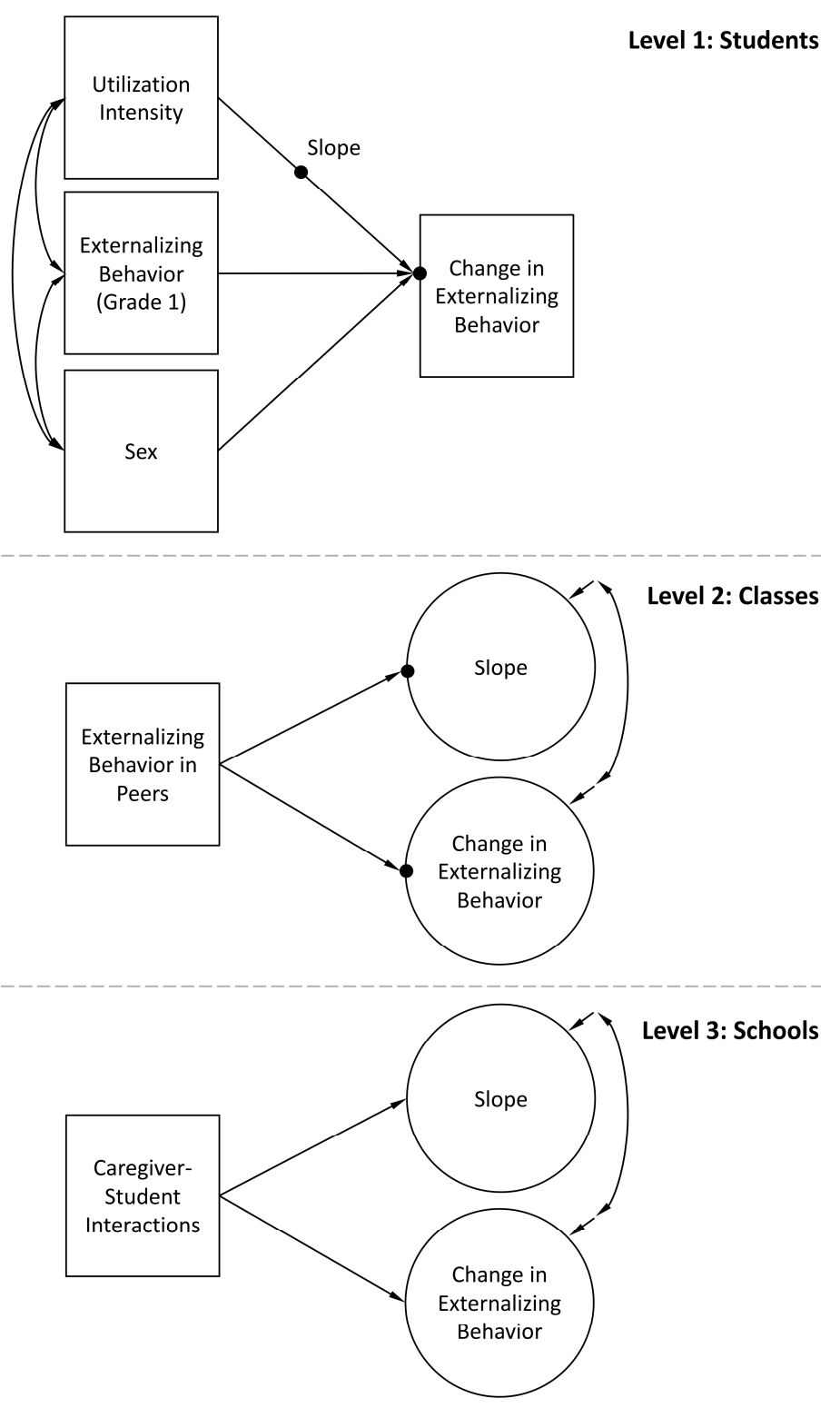




\section{Variance Partitioned at the Student, Class, and School Level}

On average, externalizing behavior decreased by 0.46 scale points from grade 1 to grade 2. Most of the variance in this change in externalizing behavior is due to student characteristics $(86.3 \%)$, with class characteristics $(8.5 \%)$ and school characteristics $(5.2 \%)$ having a smaller impact. Results for subsequent analyses are reported in table 2.

\section{Hypothesis 1: Intensity of Extended Education Utilization}

To examine a possible main effect of extended education utilization intensity on change in externalizing behavior, we added utilization intensity as a predictor on the student level, while controlling for initial levels of externalizing behavior and student's sex (Model 1). As results show, the development in externalizing behavior is more favorable for students with higher initial levels of externalizing behavior $(\mathrm{p} \leq .001)$ : A one-point increase in externalizing behavior in grade 1 is associated with a 0.20 decrease in externalizing behavior by the end of grade 2. For student's sex, a trend emerged, with female students having a 0.47 decrease in externalizing behavior $(\mathrm{p} \leq .10)$ compared to male students. In contrast, intensity of extended education utilization failed to exert a significant impact. Compared to the null-model, those three predictors explained about $9 \%$ of the variance at the student level, $11 \%$ at the class level and $19 \%$ at the school level. Model-fit also improved significantly $\left(\chi^{2}=37.92, \mathrm{df}=3, \mathrm{p} \leq .001\right)$, with both smaller log-likelihood and BIC.

\section{Hypotheses 2 and 3: Peer Group and Caregiver-Student Interactions}

Next, we tested a possible main effect of initial externalizing behavior in peers on the development of externalizing behavior by adding this variable at the class level (Model 2), although with no significant result. Similarly, we tested a possible impact of caregiver-student-interactions (Model 3) at the school level. Again, no significant main effect was found. Both model 2 and 3 did not substantially improve $\mathrm{R}^{2}$ or fit indices, compared to model 1.

\section{Hypotheses 4a and 4b: Cross-Level Interactions}

In order to test possible interactions between predictors, the slope between utilization intensity and change in externalizing behavior was allowed to vary between classes and schools and was tested for cross-level interactions. As can be seen in Model 4 , the impact of extended education utilization intensity on externalizing behavior was moderated by both initial externalizing behavior in peers and caregiver-student interactions: For a one standard-deviation increase in peers' externalizing behavior, an additional hour of extended education utilization predicted a 0.05 increase in externalizing behavior by the end of grade $2(\mathrm{p} \leq .05)$. 





Additionally, for a one standard-deviation increase in caregiver-student interaction quality, an additional hour of extended education utilization predicted a 0.04 decrease in externalizing behavior by the end of grade $2(\mathrm{p} \leq .01)$. In short: Lower levels of externalizing behavior in peers and higher quality of caregiver-student interactions were both linked to a more favorable relationship between utilization intensity and change in externalizing behavior. Compared to model 1, after adding both cross-level interactions, another $5 \%$ of the variance at the student level and another $23 \%$ at the school level was explained. Model-fit also improved, with both lower log-likelihood and AIC $\left(\chi^{2}=20.32, \mathrm{df}=8, \mathrm{p} \leq .01\right)$.

To allow for an easier interpretation of those cross-level interactions, the same model was calculated with z-standardized utilization intensity. Regression-coefficients were then used to estimate change in externalizing behavior for students with high levels ( 1 standard deviation above mean) or low levels (1 standard deviation below mean) of different characteristics (Richter, 2007). As figure 2 shows, for students with high levels of externalizing behavior in peers, higher utilization intensity seems to increase externalizing behavior. In contrast, for students with low levels of externalizing behavior in peers, higher utilization intensity seems to reduce externalizing behavior. Also in line with our hypothesis, students who experience high levels of externalizing behavior among peers seem to develop less favorably than students who experience low levels of externalizing behavior among peers, if they use extended education more intensively. However, for students with low utilization intensity, the contrary seems to be the case, even suggesting an adverse effect of lower externalizing behavior in peers.

Figure 2. Differences in students' development of externalizing behavior, based on externalizing behavior in peers ( \pm 1 SD) and utilization intensity $( \pm 1 \mathrm{SD})$, controlling for individual and school level variables.

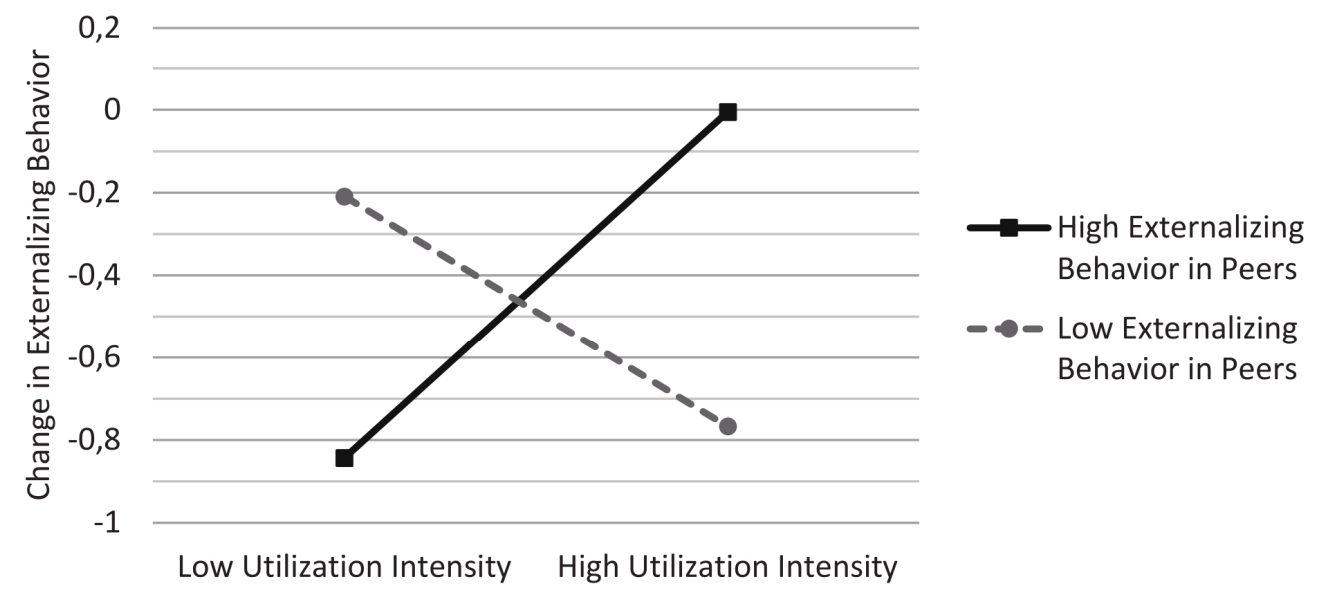

Similar results have been found with regard to caregiver-student interactions: As figure 3 indicates, higher utilization intensity decreases externalizing behavior compared to lower utilization intensity, if caregiver-student interactions have a higher quality. For schools with lower interaction quality, the contrary seems to be the case. Furthermore, students who experience high quality caregiver-student interactions seem to develop more favorably than students from schools with low quality inter- 
actions, if they use extended education more intensively. However, for students with low utilization intensity, high quality of interactions seems to have an adverse effect.

Figure 3. Differences in students' development of externalizing behavior, based on staff-student interactions ( \pm 1 SD) and utilization intensity ( \pm 1 SD), controlling for individual and school level variables.

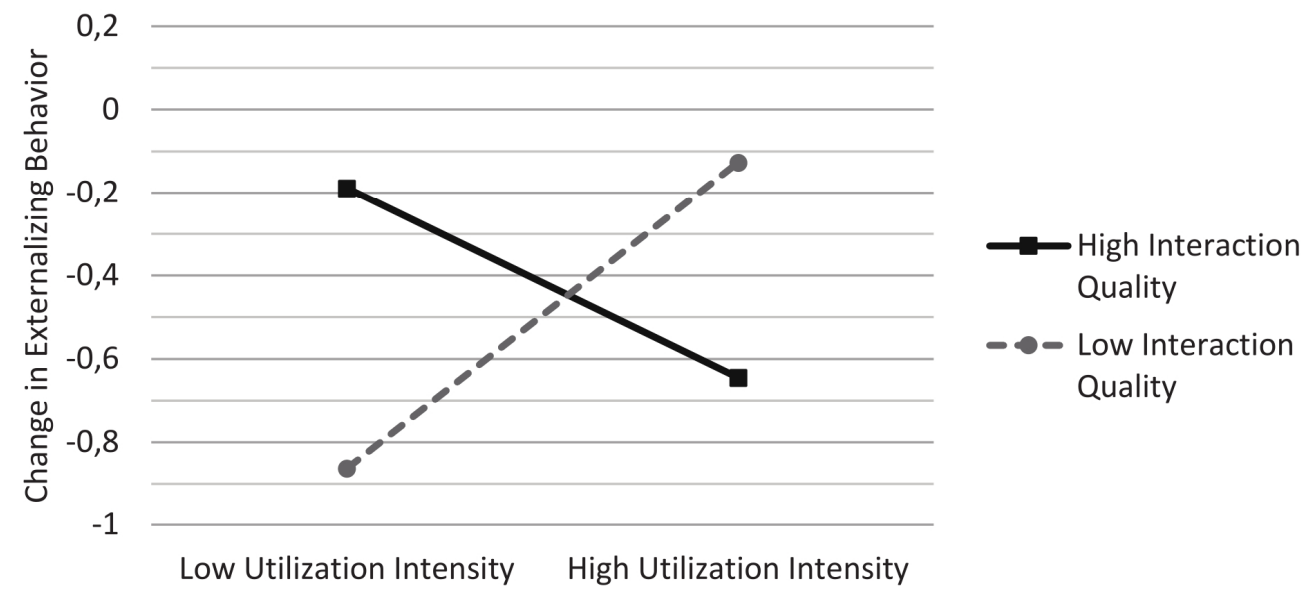

\section{Discussion}

In short, the lack of any main effects suggests that neither extended education utilization intensity, nor externalizing behavior in peers nor caregiver-student interactions alone affect change in externalizing behavior, negating hypotheses 1 to 3 . However, in line with hypotheses $4 \mathrm{a}$ and $4 \mathrm{~b}$, we did find two cross-level interactions: Higher utilization intensity was more favorably linked to the development of externalizing behavior if caregiver-student interactions had a higher quality and if initial levels of externalizing behavior in peers were lower.

Utilization intensity alone does not seem to affect the development of externalizing behavior. This result confirms findings from a previous study in Switzerland (Schüpbach et al., 2014) and is also in line with the conclusion from Roth et al. (2010). While utilization intensity as a factor may potentially provide more information and thus be helpful in explaining different findings, rather than only considering mere participation, it seems reasonable to assume that any effects of extended education settings - no matter how intensively they are used - still depend on additional characteristics.

As previous research on child contagion suggested (Dishion \& Tipsord, 2011), externalizing behavior in peers may influence the development of externalizing behavior. In our study, we could not confirm such a general notion. This may seem surprising, as students not only share extended education but also regular school hours with the respective peer group. However, regular school hours may be more firmly structured and supervised than extended education, which may counteract the possible effect of externalizing behavior within peer groups (Dishion \& Tipsord, 2011).

Contrary to findings regarding after-school programs (Pierce et al., 2010; Wade, 2015), results did not indicate caregiver-student interactions having a general effect 
on change in externalizing behavior. It is possible that overall utilization intensity was not strong enough to elicit such an effect, which would be supported by our finding of a cross-level interaction between the quality of caregiver-student interactions and utilization intensity.

To our knowledge, our contribution is the first to explore externalizing behavior in peers as a possible moderating factor of the effects of extended education. As expected, lower levels of externalizing behavior in peers predicted a more favorable relationship between utilization intensity and change in externalizing behavior: Students who experienced low levels of externalizing behavior among their peers developed more favorably, if they used extended education more intensively and the contrary was found when levels of externalizing behavior among peers were high. Consequently, for students with high utilization intensity, higher initial levels of externalizing behavior among peers were associated with an increase in externalizing behavior, compared to students with lower levels. So far, those results are in line with literature regarding peer contagion (Dishion \& Tipsord, 2011). However, the development of externalizing behavior in students with low utilization intensity also differed. Among these students, higher levels of externalizing behavior among peers predicted a more favorable outcome, that is, a decrease in externalizing behavior. This finding is difficult to explain, especially since we could not find any influential outliers. In fact, inspection of the scatterplot indicated a strong linear relationship between externalizing behavior in peers and the slope between utilization intensity and change in externalizing behavior. Since it seems unlikely, that higher levels of externalizing behavior in peers would exert a favorable influence on the development of externalizing behavior, results point to confounded variables, either at the class or at the individual level.

Similar results emerged with regard to the cross-level interaction between quality of caregiver-student interactions and utilization intensity: For students with low quality of interactions, higher utilization intensity predicted an increase in externalizing behavior. In contrast, higher utilization intensity was linked to a decrease in externalizing behavior, when quality of caregiver-student interactions was high. Consequently, higher quality caregiver-student interactions were associated with a decrease in externalizing behavior, when extended education was utilized intensively. So far, results are in line with Wade (2015) who found that an after-school program resulted in an adverse effect when caregiver-student interaction quality was low. However - analogical to the other cross-level interaction - converse results were found for students with low utilization intensity, that is, with higher quality of caregiver-student interactions being linked to adverse outcomes. Again, such an effect seems unlikely and implies confounding variables, either at the school level or at the individual level.

\section{Limitations}

While the longitudinal multilevel design of our contribution allowed us to explore possible influences of change in externalizing behavior on their respective levels, there are several limitations: (1) With an average of only about 5 students per class 
and about 10 students per school, sample size was already quite small, especially considering the small amount of variation at class- and school-levels. (2) Other relevant variables such as socioeconomic background or intelligence could not be included in analyses because they would have led to yet another reduction of sample size. (3) Ideally, the degree of externalizing behavior in peers would have been accounted for by including externalizing behavior at the end of grade 1 as latent predictor at the class level, because the simple aggregation variables of a lower cluster might bias standard errors. However, three-level models in MPlus do not allow predictors to be specified at more than one level. (4) Furthermore, the level-2 unit "classes" could refer both to a student's classmates and to his or her peers in the respective extended education setting. While consideration of externalizing behavior in all peers during extended education might be the most straightforward approach, our sample was limited to a few classes per school and did not represent all children utilizing extended education. Therefore, focusing on a student's classmates, which were also enrolled in extended education, seemed appropriate.

\section{Conclusion}

This contribution explored several possible moderators which may help to explain differential effects of extended education on externalizing behavior. Per se, utilization intensity, externalizing behavior in peers and caregiver-student interactions did not predict change in externalizing behavior. However, the effect of utilization intensity on externalizing behavior was moderated by both externalizing behavior in peers and caregiver-student interactions. While those findings generally confirm our assumption that utilization intensity has a favorable effect on externalizing behavior when the degree of externalizing behavior in peers is low and the quality of caregiver-student interactions is high, further inspection of both cross-level interactions raises caution. Further studies should reexamine those interactions in larger samples while controlling for additional characteristics at the individual, class, and school level (e.g. socioeconomic background, intelligence, quality of extended education beyond caregiver-student interactions), allowing for a more thorough inspection of possible confounders.

\section{References}

Aeberli, C., \& Binder, H.-M. (2005). Das Einmaleins der Tagesschule: Ein Leitfaden für Gemeinde- und Schulbehörden. Zürich: Avenir Suisse.

Browne, G., Gafni, A., Roberts, J., Byrne, C., \& Majumdar, B. (2004). Effective/ efficient mental health programs for school-age children: A synthesis of reviews. Social Science \& Medicine, 58(7), 1367-1384. http://dx.doi.org/10.1016/S02779536(03)00332-0 
Byrne, B. M. (2012). Structural equation modeling with Mplus: Basic concepts, applications, and programming. Multivariate applications series. New York: Routledge Taylor \& Francis Group.

Dishion, T. J., \& Tipsord, J. M. (2011). Peer contagion in child and adolescent social and emotional development. Annual Review of Psychology, 62(1), 189-214. http://dx.doi.org/10.1146/annurev.psych.093008.100412

Durlak, J. A., Weissberg, R. P., \& Pachan, M. (2010). A meta-analysis of afterschool programs that seek to promote personal and social skills in children and adolescents. American journal of community psychology, 45(3-4), 294-309. http://dx.doi.org/10.1007/s10464-010-9300-6

EDK. (2013). Kantonsumfrage 2012/2013. Retrieved from http://edudoc.ch/record/ 115194/files/2013.pdf

Fergusson, D. M., Horwood, L. J., \& Ridder, E. M. (2007). Conduct and attentional problems in childhood and adolescence and later substance use, abuse and dependence: Results of a 25-year longitudinal study. Drug and Alcohol Dependence, 88, S14-S26. http://dx.doi.org/10.1016/j.drugalcdep.2006.12.011

Fischer, N., Kuhn, H. P., \& Züchner, I. (2011). Entwicklung von Sozialverhalten in der Ganztagsschule: Wirkungen der Ganztagsteilnahme und der Angebotsqualität. In N. Fischer, H. G. Holtappels, E. Klieme, T. Rauschenbach, L. Stecher, \& I. Züchner (Eds.), Ganztagsschule: Entwicklung, Qualität, Wirkungen. Längsschnittliche Befunde der Studie zur Entwicklung von Ganztagsschulen (StEG) (pp. 246-266). Weinheim \& Basel: Beltz Juventa.

Goodman, A., Lamping, D. L., \& Ploubidis, G. B. (2010). When to use broader internalising and externalising subscales instead of the hypothesised five subscales on the Strengths and Difficulties Questionnaire (SDQ): Data from British parents, teachers and children. Journal of abnormal child psychology, 38(8), 1179-1191. http://dx.doi.org/10.1007/s10802-010-9434-x

Goodman, R. (1997). The Strengths and Difficulties Questionnaire: A research note. Journal of Child Psychology and Psychiatry, 38(5), 581-586. http://dx.doi. org/10.1111/j.1469-7610.1997.tb01545.x

Harms, T., Jacobs, E. V., \& White, D. R. (1996). School-age care environment rating scale. New York: Teachers College Press.

Heck, R. H., \& Thomas, S. L. (2015). An introduction to multilevel modeling techniques: MLM and SEM approaches using Mplus (3. ed.). New York: Routledge.

Kanevski, R., \& von Salisch, M. (2011). Fördert die Ganztagsschule die Entwicklung sozialer und emotionaler Kompetenzen bei Jugendlichen? Zeitschrift für Erziehungswissenschaft, 14(3), 237-259. http://dx.doi.org/10.1007/s11618-0110238-8

Kellam, S. G., Ling, X., Merisca, R., Brown, C. H., \& Ialongo, N. (1998). The effect of the level of aggression in the first grade classroom on the course and malleability of aggressive behavior into middle school. Development and Psychopathology, 10(2), 165-185. http://dx.doi.org/10.1017/S0954579498001564

Kremer, K. P., Maynard, B. R., Polanin, J. R., Vaughn, M. G., \& Sarteschi, C. M. (2015). Effects of after-school programs with at-risk youth on attendance and externalizing behaviors: A systematic review and meta-analysis. Journal of Youth and Adolescence, 44(3), 616-636. http://dx.doi.org/10.1007/s10964-014-0226-4 
Liu, J. (2004). Childhood externalizing behavior: Theory and implications. Journal of Child and Adolescent Psychiatric Nursing, 17(3), 93-103. http://dx.doi. org/10.1111/j.1744-6171.2004.tb00003.x

McLeod, J. D., \& Kaiser, K. (2004). Childhood emotional and behavioral problems and educational attainment. American Sociological Review, 69(5), 636-658. http://dx.doi.org/10.1177/000312240406900502

Metsäpelto, R.-L., Pakarinen, E., Kiuru, N., Poikkeus, A.-M., Lerkkanen, M.-K., \& Nurmi, J.-E. (2015). Developmental dynamics between children's externalizing problems, task-avoidant behavior, and academic performance in early school years: A 4-year follow-up. Journal of Educational Psychology, 107(1), 246-257. http://dx.doi.org/10.1037/a0037389

Muthén, L. K., \& Muthén, B. O. (1998-2012). Mplus user's guide: Seventh edition. Los Angeles: Muthén \& Muthén.

Nation, M., Crusto, C., Wandersman, A., Kumpfer, K. L., Seybolt, D., Morrissey-Kane, E., \& Davino, K. (2003). What works in prevention: Principles of effective prevention programs. American Psychologist, 58(6-7), 449-456. http://dx.doi.org/10.1037/0003-066X.58.6-7.449

O'Hare, L., Biggart, A., Kerr, K., \& Connolly, P. (2015). A randomized controlled trial evaluation of an after-school prosocial behavior program in an area of socioeconomic disadvantage. The Elementary School Journal, 116(1), 1-29. http:// dx.doi.org/10.1086/683102

Pierce, K. M., Bolt, D. M., \& Vandell, D. L. (2010). Specific features of after-school program quality: Associations with children's functioning in middle childhood. American Journal of Community Psychology, 45(3-4), 381-393. http://dx.doi. org/10.1007/s10464-010-9304-2

Richter, T. (2007). Wie analysiert man Interaktionen von metrischen und kategorialen Prädiktoren? Nicht mit Median-Splits! Zeitschrift für Medienpsychologie, 19(3), 116-125. http://dx.doi.org/10.1026/1617-6383.19.3.116

Roth, J. L., Malone, L. M., \& Brooks-Gunn, J. (2010). Does the amount of participation in afterschool programs relate to developmental outcomes? A review of the literature. American journal of community psychology, 45(3-4), 310-324. http://dx.doi.org/10.1007/s10464-010-9303-3

Schüpbach, M., Ignaczewska, J., \& Herzog, W. (2014). Sozio-emotionale Entwicklung von Ganztagsschulkindern auf der Primarschulstufe. Zeitschrift für Entwicklungspsychologie und Pädagogische Psychologie, 46(1), 11-23. http://dx.doi.org/10.1026/0049-8637/a000096

Snyder, J., Schrepferman, L., McEachern, A., Barner, S., Johnson, K., \& Provines, J. (2008). Peer deviancy training and peer coercion: Dual processes associated with early-onset conduct problems. Child Development, 79(2), 252-268. http://dx.doi.org/10.1111/j.1467-8624.2007.01124.x

Stern, S., Iten, R., Schwab, S., Felfe, C., Lechner, M., \& Thiemann, P. (2013). Familienergänzende Kinderbetreuung und Gleichstellung. Zürich, St. Gallen: INFRAS, Schweizerisches Institut für Empirische Wirtschaftsforschung, Universität St. Gallen.

Stone, L. L., Otten, R., Engels, R. C. M. E., Vermulst, A. A., \& Janssens, J. M. A. M. (2010). Psychometric properties of the parent and teacher versions of 
the Strengths and Difficulties Questionnaire for 4- to 12-year-olds: A review. Clinical Child and Family Psychology Review, 13(3), 254-274. http://dx.doi. org/10.1007/s10567-010-0071-2

Tietze, W., Rossbach, H.-G., Stendel, M., \& Wellner, B. (2007). Hort- und Ganztagsangebote-Skala (HUGS): Feststellung und Unterstützung pädagogischer Qualität in Horten und außerunterrichtlichen Angeboten. Berlin: Cornelsen Scriptor.

Wade, C. E. (2015). The longitudinal effects of after-school program experiences, quantity, and regulatable features on children's social-emotional development. Children and Youth Services Review, 48, 70-79. http://dx.doi.org/10.1016/j. childyouth.2014.12.007

Warren, K., Schoppelrey, S., Moberg, D. P., \& McDonald, M. (2005). A model of contagion through competition in the aggressive behaviors of elementary school students. Journal of Abnormal Child Psychology, 33(3), 283-292. http://dx.doi.org/10.1007/s10802-005-3565-5

Woerner, W., Becker, A., \& Rothenberger, A. (2004). Normative data and scale properties of the German parent SDQ. European Child \& Adolescent Psychiatry, 13(S2), 4-10. http://dx.doi.org/10.1007/s00787-004-2002-6 


\section{Manuskript 3}

Frei, L., Schüpbach, M., von Allmen, B. \& Nieuwenboom, W. (2021). Außerunterrichtliche Bildungs- und Betreuungsangebote als Mittel gegen externalisierendes Verhalten? Die Bedeutung der Gleichaltrigen und des Betreuungspersonals an Deutschschweizer Tagesschulen. Psychologie in Erziehung und Unterricht, 68 (2), 117-134. http://dx.doi.org/10.2378/ peu2021.art06d 
Außerunterrichtliche Bildungs- und Betreuungsangebote als Mittel gegen externalisierendes Verhalten? Die Bedeutung der Gleichaltrigen und des Betreuungspersonals an Deutschschweizer Tagesschulen.

\title{
Lukas Frei, Marianne Schüpbach, Benjamin von Allmen \& Wim Nieuwenboom
}

\begin{abstract}
Kann die Nutzung von außerunterrichtlichen Bildungs- und Betreuungsangeboten dazu beitragen, externalisierendes Verhalten zu reduzieren? Die bisherige Forschung zeigt widersprüchliche Befunde und rückt damit mögliche Moderatoren in den Vordergrund. Anhand einer Stichprobe von 1596 Schülerinnen und Schülern aus 113 Klassen und 52 offenen Tagesschulen in der Deutschschweiz untersucht dieser Beitrag die Entwicklung von externalisierendem Verhalten bei Schülerinnen und Schülern mit oder ohne Nutzung des Tagesschulangebots. Dabei wird berücksichtigt, dass sich Tagesschulangebote sowohl in der jeweils vorhandenen Ausprägung des externalisierenden Verhaltens der Gleichaltrigen als auch in der Interaktionsqualität mit dem Betreuungspersonal unterscheiden. Mehrebenenanalysen zeigen insgesamt eine Abnahme von externalisierendem Verhalten zwischen Ende erste und Ende zweite Jahrgangsstufe, die allerdings durch eine Nutzung des Tagesschulangebots abgeschwächt wird. Weitere Analysen liefern Hinweise, dass das externalisierende Verhalten der Gleichaltrigen und die Interaktionsqualität mit dem Betreuungspersonal eine moderierende Rolle spielen.
\end{abstract}

Schlüsselwörter: Außerunterrichtliche Bildung und Betreuung, Ganztagsschulen, Externalisierendes Verhalten, Peers, Interaktionsqualität Volltext verfügbar unter http://dx.doi.org/10.2378/peu2021.art06d 


\section{Manuskript 4}

Frei, L., Schüpbach, M., Nieuwenboom, W. \& Allmen, B. von (2018). Sozio-emotionale Entwicklung. In M. Schüpbach, L. Frei \& W. Nieuwenboom (Hrsg.), Tagesschulen (S. 213-228). Wiesbaden: Springer Fachmedien Wiesbaden. https://doi.org/10.1007/978-3-658-19024-8

Reprinted by permission from Springer Nature Customer Service Centre GmbH. 


\title{
Sozio-emotionale Entwicklung
}

\author{
Lukas Frei, Marianne Schüpbach, Wim Nieuwenboom und \\ Benjamin von Allmen
}

\section{Einleitung}

Nebst der Förderung von schulischen Leistungen stellen insbesondere das soziale Lernen und damit verbunden die Förderung von sozialer Kompetenz eine zentrale Erwartung an Tagesschulen ${ }^{1}$ dar (vgl. Was ist eine Tagesschule? - eine historische Herleitung der Zeitorganisation an Schulen und eine begriffliche Klärung in diesem Band). Kanning (2002) definiert soziale Kompetenz als „Gesamtheit des Wissens, der Fähigkeiten und Fertigkeiten einer Person, welche die Qualität eigenen Sozialverhaltens [...] fördert" (S. 155). Dabei kann sich soziale Kompetenz sowohl auf konkrete Verhaltensweisen beziehen, als auch auf Wahrnehmungen, Kognitionen, Motive oder Emotionen (Kanning, 2002). Geringe soziale Kompetenz findet ihren Ausdruck in Verhaltensauffälligkeiten, wobei meist zwischen internalisierendem und externalisierendem Verhalten unterschieden wird. Internalisierendes Verhalten wird dabei durch „Rückzug und Selbsteinschränkung" gekennzeichnet, während sich externalisierendes Verhalten auf ein „Agieren nach außen (z. B. ein besonderes Maß an Aggressivität)“ bezieht (Fröhlich-Gildhoff, 2013, S. 39). Ein Kontrastpunkt dazu stellt prosoziales Verhalten dar, welches sich durch eine stärkere Berücksichtigung fremder Interessen auszeichnet (Kanning, 2002).

Externalisierendes Verhalten während den ersten Schuljahren verringert die Wahrscheinlichkeit, einen Hochschulabschluss zu erlangen (McLeod \& Kaiser, 2004), wirkt sich nachteilhaft auf schulische Leistungen aus (Metsäpelto, Pakarinen, Kiuru, Poikkeus, Lerkkanen \& Nurmi, 2015) und kann zu Suchtproblemen führen (Fergusson, Horwood \& Ridder, 2007). Internalisierendes Verhalten wäh-

\footnotetext{
1 Als Tagesschulen werden in diesem Kapitel Schulen bezeichnet, die an mehreren Tagen pro Woche über den traditionellen Unterricht hinaus ganztägige Bildungs- und Betreuungsangebote (inklusive Mittagsverpflegung) anbieten (EDK, 2013). An gebundenen Tagesschulen ist das Tagesschulangebot mit Ausnahme sogenannter Auffangzeiten für alle Schülerinnen und Schüler verpflichtend, an offenen Tagesschulen stehen modular aufgebaute Angebote zur Verfügung, die freiwillig genutzt werden können.
} 
rend der Schulzeit erhöht die Wahrscheinlichkeit von Gewalterfahrungen durch Gleichaltrige (Reijntjes, Kamphuis, Prinzie \& Telch, 2010) und wirkt sich ebenso wie übrigens externalisierendes Verhalten -durch erhöhte Entzündungswerte (Slopen, Kubzansky \& Koenen, 2013) ungünstig auf die physische Gesundheit aus. Im Gegensatz dazu fördert prosoziales Verhalten positive Emotionen (Nelson, Layous, Cole \& Lyubomirsky, 2016) und kann einer Viktimisierung durch Gleichaltrige entgegenwirken (Leadbeater, Thompson \& Sukhawathanakul, 2016). Selbst geringe Ausprägungen von internalisierendem und externalisierendem Verhalten, oder ein Mangel an prosozialem Verhalten, erhöhen die Wahrscheinlichkeit, bestimmte psychische Störungen zu entwickeln (Goodman, Lamping \& Ploubidis, 2010).

Angesichts der Bedeutung von sozialer Kompetenz ist eine frühe Förderung naheliegend. $\mathrm{Ob}$ diese durch eine Teilnahme am Tagesschulangebot gelingt, ist allerdings kaum untersucht und gesichert. Bezüglich Tagesschulen in der Deutschschweiz wurde dies von Schüpbach (2010) auf der Primarschulstufe untersucht. Die Stichprobe umfasste 487 Primarschülerinnen und -schüler, wobei ein Teil der Schülerschaft ein offenes oder ein gebundenes Tagesschulangebot nutzte. Eine erste Analyse zeigte einen positiven Effekt der Angebotsnutzung auf die Entwicklung von sozio-emotionalen Verhaltensstärken und prosozialem Verhalten zwischen Ende der ersten und Ende der zweiten Jahrgangsstufe. In einer vertiefenden Analyse, in der einerseits der Untersuchungszeitraum bis Ende der dritten Jahrgangsstufe ausgeweitet wurde und andererseits auf Geschlecht, IQ und familiale Ressourcen hin kontrolliert wurde, zeigte die Nutzung des Tagesschulangebots allerdings keinen signifikanten Einfluss mehr (Schüpbach, Ignaczewska \& Herzog, 2014). Befunde zur Angebotsnutzung an Ganztagsschulen in Deutschland fallen uneinheitlich aus, wobei teils positive, teils keine und teils negative Effekte einer Angebotsteilnahme auf die Entwicklung der sozialen Kompetenz gefunden wurden (Fischer, Brümmer \& Kuhn, 2011; Fischer, Kuhn \& Züchner, 2011; Kanevski \& von Salisch, 2011). Dasselbe gilt für ausserunterrichtliche Bildungs- und Betreuungsangebote in den USA, sogenannte ,afterschool programs“" (Durlak, Weissberg \& Pachan, 2010; Kremer, Maynard, Polanin, Vaughn \& Sarteschi, 2015). Allerdings sind die meisten bisherigen Befunde mit der Einschränkung verbunden, dass die Klassen- und Schulzugehörigkeit nicht oder nur teilweise berücksichtigt wurden, was zu verzerrten Parameterschätzungen führen kann (Van den Noortgate, Opdenakker \& Onghena, 2005). In ersten Analysen zu unserer aktuellen Stichprobe von Primarschülerinnen und schülern aus offenen Tagesschulen zeigte sich, unter Kontrolle der Mehrebenen- 
struktur, dass die Angebotsnutzung hinsichtlich der Entwicklung von externalisierendem Verhalten einen unvorteilhaften Einfluss ausübt (Frei, Schüpbach, von Allmen \& Nieuwenboom, 2017).

Angesichts der Heterogenität in den bisherigen Studienergebnissen stellt sich die Frage, unter welchen Umständen positive oder gar negative Effekte durch eine Nutzung von Tagesschulangeboten eintreten. Dabei könnte insbesondere den Interaktionen zwischen Betreuungspersonal und Schülerschaft, als auch dem Einfluss durch die Gleichaltrigen, eine zentrale Rolle zukommen (Frei, Schüpbach, Nieuwenboom \& von Allmen, 2016). So konnten Frei et al. (2016) für externalisierendes Verhalten zeigen, dass insbesondere bei einer hohen Interaktionsqualität durch das Betreuungspersonal und bei einem niedrigen Niveau an externalisierendem Verhalten im Tagesschulangebot positive Effekte zu erwarten sind. Diese Befunde sollen nun hinsichtlich internalisierendem Verhalten und prosozialem Verhalten ergänzt werden. Nebst einer generellen Wirkung durch die Nutzung des offenen Tagesschulangebots auf die Entwicklung von Ende 1. bis Ende 2. Jahrgangsstufe sollen deshalb die Interaktionsqualität und das Verhalten bei Gleichaltrigen als mögliche Moderatoren untersucht werden. Entsprechend werden folgende Hypothesen formuliert. Hinsichtlich der Entwicklung von internalisierendem Verhalten ist eine Abnahme des Verhaltens und entsprechend ein negativer (hemmender) Effekt der Angebotsnutzung wünschenswert:

1a Die Nutzung des Tagesschulangebots beeinflusst die Entwicklung des internalisierenden Verhaltens (verstärkender oder hemmender Effekt).

$1 \mathrm{~b}$ Sofern internalisierendes Verhalten bei Gleichaltrigen stärker ausgeprägt ist, wirkt sich die Angebotsnutzung zunehmend positiv auf das internalisierende Verhalten aus (d.h. ein hemmender Effekt nimmt ab oder ein verstärkender Effekt nimmt zu).

1c Bei einem Anstieg der Interaktionsqualität wird die Wirkung der Angebotsnutzung auf das internalisierende Verhalten zunehmend negativ (d.h. ein hemmender Effekt nimmt zu oder ein verstärkender Effekt nimmt ab).

Analog werden Hypothesen für die Entwicklung des prosozialen Verhaltens formuliert. Im Gegensatz zur Entwicklung des internalisierenden Verhaltens ist hier eine Zunahme des Verhaltens und entsprechend ein positiver (verstärkender) Effekt der Angebotsnutzung wünschenswert: 
2a Die Nutzung des Tagesschulangebots beeinflusst die Entwicklung des prosozialen Verhaltens (verstärkender oder hemmender Effekt).

$2 \mathrm{~b}$ Sofern prosoziales Verhalten bei Gleichaltrigen stärker ausgeprägt ist, wirkt sich die Angebotsnutzung zunehmend positiv auf das prosoziale Verhalten aus (d.h. ein hemmender Effekt nimmt ab oder ein verstärkender Effekt nimmt zu).

2c Bei einem Anstieg der Interaktionsqualität wirkt sich die Angebotsnutzung zunehmend positiv auf das prosoziale Verhalten aus (d.h. ein hemmender Effekt nimmt ab oder ein verstärkender Effekt nimmt zu).

\section{Methode}

\subsection{Stichprobe}

Die Daten wurden zwischen 2014 und 2015 im Rahmen der Längsschnittstudie EduCare-TaSe - Tagesschulen und Schulerfolg? erhoben, die durch den Schweizerischen Nationalfonds finanziert wird. EduCare-TaSe untersucht Kinder im Grundschulalter (Ende 1. bis Ende 2. Jahrgangsstufe) an offenen Tagesschulen, d.h. ein Teil der Schülerinnen und Schüler nutzt neben dem Unterricht freiwillig zusätzlich das Tagesschulangebot. Es wurde eine Vollerhebung angestrebt, wobei aus forschungsökonomischen Gründen mindestens zwei parallele Erstjahrgangsklassen vorausgesetzt wurden. Von den insgesamt 251 angefragten offenen Tagesschulen erklärten sich 53 Schulen aus 13 Deutschschweizer Kantonen bereit, an der Studie teilzunehmen. Nach Ausschluss von Schülerinnen und Schülern mit fehlenden Werten, verblieb eine Stichprobe von insgesamt 1'185 Schülerinnen und Schülern mit vollständigen Daten. Da es unsere Absicht war, nur Schülerinnen und Schülern mit einer dauerhaften Nutzung des Tagesschulangebots, d.h. über ein Schuljahr hinweg und ohne Unterbrechung, mit Schülerinnen und Schüler ohne Nutzung des Tagesschulangebots zu vergleichen, wurden zusätzlich 109 Schülerinnen und Schüler, welche die Angebote nur über ein halbes Jahr nutzten, ausgeschlossen. Damit verbleibt eine Stichprobe von 1'076 Schülerinnen und Schülern aus 84 Klassen und 47 Schulen. Deskriptive Angaben zur Stichprobe können der Tab. 1 entnommen werden. 
Tab. 1: $\quad$ Deskriptive Statistik

\begin{tabular}{lllll}
\hline & Min & Max & $M$ & SD \\
\hline Schülerin / Schüler (N=1'076) & & & & \\
$\Delta$ Internalisierendes Verhalten & -12.00 & 13.00 & -0.31 & 2.63 \\
$\Delta$ Prosoziales Verhalten & -14.00 & 14.00 & 0.57 & 3.67 \\
Ausgangsniveau Internalisierendes Verhalten & 0.00 & 16.00 & 3.10 & 3.27 \\
Ausgangsniveau Prosoziales Verhalten & 2.00 & 20.00 & 15.54 & 4.35 \\
Kognitive Grundfähigkeiten & 24.00 & 78.00 & 52.53 & 9.20 \\
Geschlecht (1 = weiblich) & 0.00 & 1.00 & - & - \\
$\quad$ Angebotsnutzung (1 = dauerhafte Nutzung) & 0.00 & 1.00 & - & - \\
Klasse ( $N=84)$ & & & & \\
$\quad$ Internalisierendes Verhalten bei Gleichaltrigen & 0.00 & 11.00 & 3.35 & 2.40 \\
$\quad$ Prosoziales Verhalten bei Gleichaltrigen & 7.00 & 20.00 & 15.33 & 3.27 \\
Schule ( $N=47)$ & & & & \\
$\quad$ Interaktionsqualität & 1.50 & 6.00 & 4.55 & 0.90 \\
\hline
\end{tabular}

Skalierung: $\Delta$ Internalisierendes Verhalten / Prosoziales Verhalten (Skala von -20 bis +20 ), Ausgangsniveau Internalisierendes Verhalten / Prosoziales Verhalten (Skala von 0 bis 20), Kognitive Grundfähigkeiten (T-Werte, standardisiert an Normierungsstichprobe), Geschlecht ( $0=$ männlich, $1=$ weiblich), Angebotsnutzung $(0=$ keine Nutzung, $1=$ dauerhafte Nutzung), Internalisierendes Verhalten / Prosoziales Verhalten bei Gleichaltrigen (Skala von 0 bis 20), Interaktionsqualität (Skala von 0 bis 6)

\subsection{Abhängige Variablen}

Um das internalisierende Verhalten sowie das prosoziale Verhalten der Schülerinnen und Schüler zu erheben, wurde den Lehrpersonen Ende 1. und Ende 2. Jahrgangsstufe die deutschsprachige Version des Strengths and Difficulties Questionnaire (SDQ; Goodman, 1997, 2001) als online-Fragebogen vorgegeben. Dabei wurden die Lehrpersonen gebeten, jedes Item hinsichtlich des Verhaltens eines Kindes während den vergangenen 6 Monaten auf einer dreistufigen Skala zu bewerten $(0=$ "Nicht zutreffend", $2=$ "Eindeutig zutreffend"). Der SDQ unterscheidet zwischen externalisierendem, internalisierendem und prosozialem Verhalten, wobei für diesen Beitrag die Skalen internalisierendes Verhalten (10 Items, $\alpha=.81$ für beide Jahrgangsstufen) und prosoziales Verhalten (5 Items, $\alpha=$ .84 für beide Jahrgangsstufen) verwendet werden. Als abhängige Variable wurde 
jeweils der Differenzwert zwischen den zwei Messzeitpunkten gebildet, wobei Werte von -20 bis +20 möglich waren. Positive Werte zeigen eine Zunahme, negative Werte eine Abnahme des Verhaltens.

\subsection{Unabhängige Variablen}

Angaben zur Angebotsnutzung für das 1. und 2. Schuljahr wurden jeweils bei den Leiterinnen und Leitern der Tagesschulangebote eingeholt. Schülerinnen und Schüler mit einer geringen Nutzungsdauer werden von den vorliegenden Analysen ausgeschlossen, stattdessen werden Schülerinnen und Schüler ohne Nutzung $(74 \%$, Angebotsnutzung $=0)$ und jene mit einer dauerhaften Angebotsnutzung während zwei Schuljahren $(26 \%$, Angebotsnutzung $=1)$ verglichen. Um das internalisierende Verhalten bei Gleichaltrigen und das prosoziale Verhalten bei Gleichaltrigen im Tagesschulangebot zu erfassen, wurden die Ende 1. Jahrgangsstufe erhobenen Lehrereinschätzungen zu den Schülerinnen und Schülern, welche dauerhaft am Tagesschulangebot teilnahmen, auf Klassenebene aggregiert. Höhere Werte stellen dabei eine stärkere Ausprägung von internalisierendem respektive prosozialem Verhalten dar. Die Interaktionsqualität wurde Ende der 1. Jahrgangsstufe eingeschätzt, wobei geschulte Mitglieder des Forschungsteams während jeweils mindestens vier Stunden Beobachtungen in den Tagesschulangeboten durchführten. Die Einschätzung erfolgte mittels der „Hortund Ganztagsangebote-Skala" (HUGS; Tietze, Roßbach, Stendel \& Wellner, 2007), einer Adaption der "School-Age Care Environment Rating Scale“ (Harms, Jacobs \& White, 1996). HUGS erfasst fünfzig Merkmale, wovon sechs Merkmale Interaktionen zwischen dem Personal und den Schülerinnen und Schülern betreffen (z.B. Beaufsichtigung, Verhaltensregeln oder Kommunikation). Für jedes Merkmal sind Werte zwischen 0 (ungenügende Qualität) und 6 (exzellente Qualität) möglich. Die sechs Merkmale zur Interaktionsqualität wurden zu einer Skala zusammengefasst $(\alpha=.71)$, mit möglichen Werten von 0 bis 6.

\subsection{Kontrollvariablen}

Um das Ausgangsniveau der einzelnen Schülerinnen und Schüler zu kontrollieren, wurde das internalisierende respektive das prosoziale Verhalten Ende der 
ersten Jahrgangsstufe als Kontrollvariable berücksichtigt. Die kognitiven Grundfähigkeiten der Schülerinnen und Schüler wurden mit der revidierten Fassung des Grundintelligenztests (CFT 1-R; Weiß \& Osterland, 2013) erhoben, wobei höhere Werte eine höhere Intelligenz bedeuten. Als weitere Kontrollvariable wurde das Geschlecht der Schülerinnen und Schüler via Klassenlisten sowie einer schriftlichen Befragung der Schülerinnen und Schüler erhoben und als männlich $(48 \%$, Geschlecht $=0)$ oder weiblich $(52 \%$, Geschlecht $=1)$ codiert .

\subsection{Statistisches Vorgehen}

Die statistischen Analysen wurden mit den Statistikprogrammen SPSS 24 (IBM Corp. 2015) und Mplus 7.3 (Muthén \& Muthén, 1998-2012) durchgeführt. Um mögliche Einflüsse auf die Entwicklung des internalisierenden Verhaltens (respektive des prosozialen Verhaltens) zu untersuchen, wurden jeweils Mehrebenenmodelle gerechnet, wobei zwischen den Ebenen der Schülerinnen und Schüler (Ebene 1), der Klassen (Ebene 2) und der Schulen (Ebene 3) unterschieden wurde. Unter Kontrolle des Ausgangsniveaus, der kognitiven Grundfähigkeit und des Geschlechts wurde im Modell 1 der Einfluss einer dauerhaften Angebotsnutzung getestet. Im Modell 2 wurden zusätzlich das internalisierende Verhalten bei Gleichaltrigen (respektive das prosoziale Verhalten bei Gleichaltrigen) auf Klassenebene sowie die Interaktionsqualität auf Schulebene als Prädiktoren hinzugefügt. Im Modell 3 wurde schliesslich mittels Cross-Level-Interaktionen getestet, ob der Einfluss der dauerhaften Angebotsnutzung auf die Entwicklung des internalisierenden Verhaltens (respektive des prosozialen Verhaltens) durch das Verhalten der Gleichaltrigen oder die Interaktionsqualität moderiert wurde. Als statistisches Verfahren zur Modellschätzung und Modellbewertung wurde das „Robuste Maximum-Likelihood-Schätzverfahren“ (MLR) verwendet. Sämtliche Prädiktoren wurden am Gesamtmittelwert zentriert und mit Ausnahme der dichotomen Variablen z-standardisiert. Da Mplus 7.3 bei Modellen mit Cross-LevelInteraktionen (intercept-and-slopes-as-outcomes) kein $\mathrm{R}^{2}$ berechnet, werden Loglikelihood und AIC berichtet, wobei niedrigere Werte einen besseren Modell-Fit bedeuten (Byrne, 2012). Wie von Muthén und Muthén (1998-2012) vorgeschlagen, wurde zudem mithilfe von Loglikelihood und Skalierungskorrektur ein robuster Chi-Quadrat Test durchgeführt, um die Modelle zu vergleichen. Der $\alpha$-Fehler wurde bei $5 \%$ festgelegt. Sofern es sich um gerichtete Hypothesen handelt (Hypothesen 1b, 1c, 2b und 2c) wurde einseitig getestet. 


\section{Ergebnisse}

\subsection{Internalisierendes Verhalten}

Im Durchschnitt verringerte sich internalisierendes Verhalten von Ende der ersten Jahrgangsstufe bis Ende der zweiten Jahrgangsstufe um 0.31 Skalenpunkte. Der grösste Teil der Varianz wird dabei durch die Ebene der Schülerinnen und Schüler erklärt (89\%), während die Klassenebene (10\%) und insbesondere die Schulebene (1\%) deutlich weniger zur Varianzaufklärung beitragen.

Im Modell 1 (Tab. 2) wurden das Ausgangsniveau, die kognitiven Grundfähigkeiten, das Geschlecht sowie die Angebotsnutzung als Prädiktoren aufgenommen. Die Angebotsnutzung zeigte dabei einen signifikant positiven Einfluss auf die Entwicklung des internalisierenden Verhaltens $(B=.30, \mathrm{p} \leq .05)$, wobei Schülerinnen und Schüler mit einer dauerhaften Nutzung des Tagesschulangebots im Vergleich zu Schülerinnen und Schülern ohne Angebotsnutzung eine geringere Abnahme von internalisierendem Verhalten aufwiesen (Hypothese 1a). Im Modell 2 wurden zusätzlich das internalisierende Verhalten bei Gleichaltrigen auf der Klassenebene und die Interaktionsqualität auf Schuleben als Prädiktoren aufgenommen, wobei sich für das internalisierende Verhalten der Gleichaltrigen ein signifikant positiver Effekt zeigte $(B=.49, \mathrm{p} \leq .001)$. Im Modell 3 wurden schliesslich die Interaktionen der Angebotsnutzung mit dem internalisierenden Verhalten bei Gleichaltrigen (Ebene 2), sowie mit der Interaktionsqualität (Ebene 3), als Prädiktoren hinzugefügt. Dabei zeigte sich ein signifikant positiver Interaktionseffekt zwischen internalisierendem Verhalten bei Gleichaltrigen und der Angebotsnutzung $(B=.41, \mathrm{p} \leq .01)$, der auch in Abb. 1 dargestellt ist: Sofern das internalisierende Verhalten bei Gleichaltrigen nur schwach ausgeprägt war (1 Standardabweichung unter dem Mittelwert), zeigte sich kaum ein Unterschied zwischen den Schülerinnen und Schülern mit oder ohne dauerhafte Angebotsnutzung. Bei einer hohen Ausprägung des internalisierenden Verhaltens bei Gleichaltrigen (1 Standardabweichung über dem Mittelwert) kam es bei den Schülerinnen und Schülern mit einer dauerhaften Nutzung, verglichen mit den Schülerinnen und Schülern ohne Angebotsnutzung, zu einer Zunahme von internalisierendem Verhalten und damit zu einer unvorteilhaften Entwicklung (Hypothese 1b). Für die Interaktionsqualität konnte hingegen keine Interaktion mit der Angebotsnutzung festgestellt werden (Hypothese 1c). 
Tab. 2: Mehrebenenmodelle zur Vorhersage der Entwicklung von internalisierendem Verhalten

\begin{tabular}{|c|c|c|c|}
\hline Parameter & Modell 1 & Modell 2 & Modell 3 \\
\hline Intercept & $-.33 *(.14)$ & $-.33^{* *}(.12)$ & $-.33 * *(.12)$ \\
\hline \multicolumn{4}{|l|}{ Ebene 1: Schülerin / Schüler } \\
\hline Ausgangsniveau & $-1.35 * * *(.09)$ & $-1.39 * * *(.09)$ & $-1.42 * * *(.09)$ \\
\hline Kognitive Grundfähigkeiten & $-.25 * *(.08)$ & $-.24 * *(.08)$ & $-.24 * *(.08)$ \\
\hline Geschlecht $(1=$ weiblich $)$ & $.09(.16)$ & $.09(.16)$ & $.10(.16)$ \\
\hline Angebotsnutzung ( 1 = dauerhaft) & $.30 *(.14)$ & $.30 *(.14)$ & $.32 *(.17)$ \\
\hline \multicolumn{4}{|l|}{ Ebene 2: Klasse } \\
\hline $\begin{array}{l}\text { Internalisierendes Verhalten bei } \\
\text { Gleichaltrigen }\end{array}$ & & $.49 * * *(.15)$ & $.54 * * *(.17)$ \\
\hline $\begin{array}{l}\text { Internalisierendes Verhalten bei } \\
\text { Gleichaltrigen } \times \text { Angebotsnutzung }\end{array}$ & & & $.41 * *(.16)$ \\
\hline \multicolumn{4}{|l|}{ Ebene 3: Schule } \\
\hline Interaktionsqualität & & $-.07(.13)$ & $-.08(.14)$ \\
\hline Interaktionsqualität $\times$ Angebotsnutzung & & & $-.01(.14)$ \\
\hline Fit Indices & Modell 1 & Modell 2 & Modell 3 \\
\hline Loglikelihood & -2398.75 & -2391.33 & -2388.97 \\
\hline AIC & 4813.49 & 4802.67 & 4805.94 \\
\hline$\Delta \chi^{2}$ & $\begin{array}{r}233.87^{* * *}(4 \\
\mathrm{df})\end{array}$ & $11.93 * *(2 \mathrm{df})$ & $3.12(4 \mathrm{df})$ \\
\hline
\end{tabular}

Anmerkungen: $\mathrm{N}=1076$ (Schülerinnen und Schüler) 84 (Klassen) 47 (Schulen); unstandardisierte B Koeffizienten (SE) ${ }^{+} \mathrm{p} \leq .10, * \mathrm{p} \leq .05, * * \mathrm{p} \leq .01,{ }^{* * *} \mathrm{p} \leq .001$ 


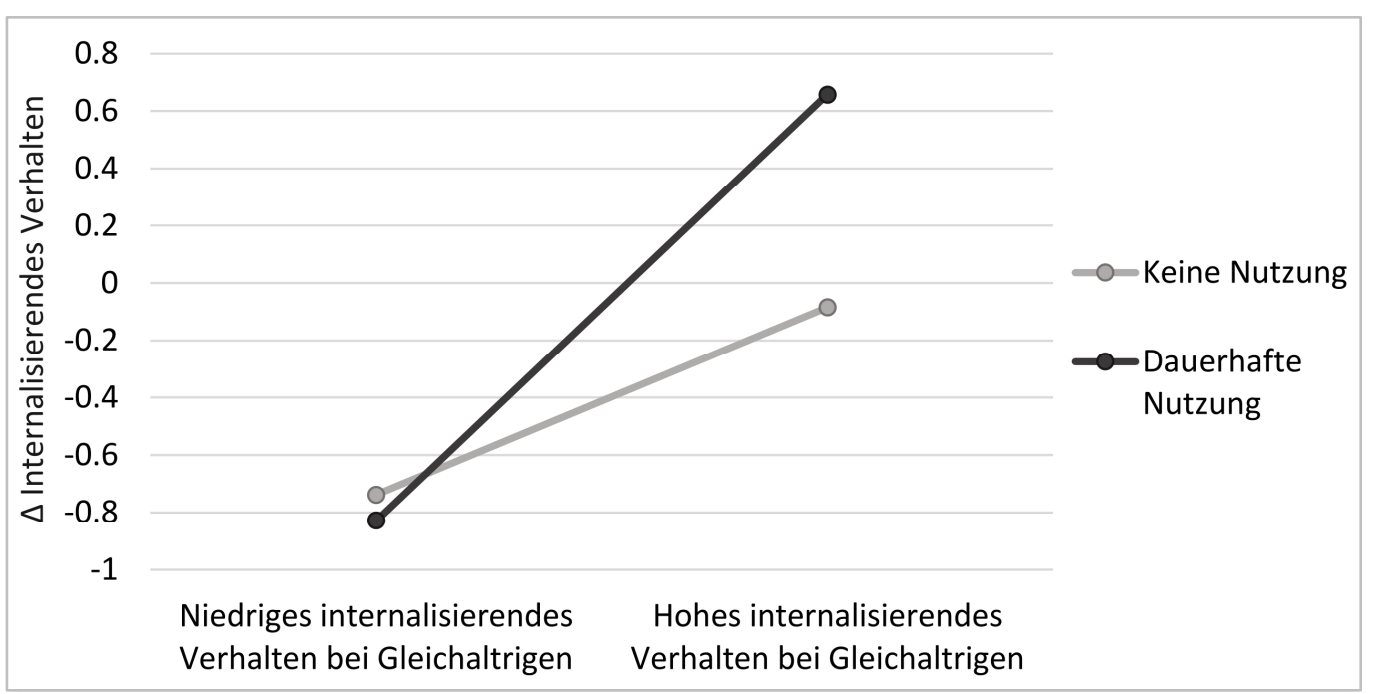

Abb. 1: Entwicklung des internalisierenden Verhaltens in Abhängigkeit der Angebotsnutzung und des internalisierenden Verhaltens bei Gleichaltrigen $( \pm 1$ SD)

\subsection{Prosoziales Verhalten}

Beim prosozialen Verhalten kam es im Schnitt zu einer Zunahme um 0.57 Skalenpunkte zwischen Ende der ersten und zweiten Jahrgangsstufe. Auch hier geht der grösste Teil der Varianz auf die Ebene der Schülerinnen und Schüler zurück (79\%), die Klassenebene zeigt aber einen deutlich stärkeren Einfluss als beim internalisierenden Verhalten (20\%). Die Schulebene trägt auch bei der Entwicklung des prosozialen Verhaltens kaum zur Varianzaufklärung bei (1\%). Das statistische Vorgehen gestaltet sich analog zum internalisierenden Verhalten: In Modell 1 (Tab. 3) wurden das Ausgangsniveau, die kognitiven Grundfähigkeiten, das Geschlecht sowie die Angebotsnutzung als Prädiktoren aufgenommen. Die Angebotsnutzung zeigte keinen signifikanten Effekt auf die Entwicklung des prosozialen Verhaltens (Hypothese 2a). In Modell 2 wurden zusätzlich das prosoziale Verhalten bei Gleichaltrigen auf der Klassenebene und die Interaktionsqualität auf Schulebene als Prädiktoren aufgenommen, allerdings zeigte sich auch hier kein signifikanter Effekt.

In Modell 3 wurden schliesslich die Interaktionen der Angebotsnutzung mit dem prosozialen Verhalten bei Gleichaltrigen (Ebene 2), sowie mit der Interaktionsqualität (Ebene 3), als Prädiktoren hinzugefügt. Keine der beiden Interaktionen zeigte einen signifikanten Effekt auf die Entwicklung des prosozialen Verhaltens (Hypothesen 2b \& 2c). 
Tab. 3: Mehrebenenmodelle zur Vorhersage der Entwicklung von prosozialem Verhalten

\begin{tabular}{|c|c|c|c|}
\hline Parameter & Modell 1 & Modell 2 & Modell 3 \\
\hline Intercept & $.53 * *(.19)$ & $.52 * *(.18)$ & $.52 * *(.18)$ \\
\hline \multicolumn{4}{|l|}{ Ebene 1: Schülerin / Schüler } \\
\hline Ausgangsniveau & $-1.93 * * *(.14)$ & $-1.94 * * *(.14)$ & $-1.92 * * *(.14)$ \\
\hline Kognitive Grundfähigkeiten & $.03(.09)$ & $.03(.08)$ & $.03(.09)$ \\
\hline Geschlecht (1 = weiblich $)$ & $.87 * * *(.22)$ & $.88 * * *(.22)$ & $.87 * * *(.22)$ \\
\hline Angebotsnutzung (1 = dauerhaft) & $-.24(.22)$ & $-.24(.22)$ & $-.26(.23)$ \\
\hline \multicolumn{4}{|l|}{ Ebene 2: Klasse } \\
\hline $\begin{array}{l}\text { Prosoziales Verhalten bei } \\
\text { Gleichaltrigen }\end{array}$ & & $.10(.21)$ & $.09(.21)$ \\
\hline $\begin{array}{l}\text { Prosoziales Verhalten bei } \\
\text { Gleichaltrigen } \times \text { Angebotsnutzung }\end{array}$ & & & $-.14(.25)$ \\
\hline \multicolumn{4}{|l|}{ Ebene 3: Schule } \\
\hline Interaktionsqualität & & $.23(.17)$ & $.23(.16)$ \\
\hline Interaktionsqualität $\times$ Angebotsnutzung & & & $.13(.20)$ \\
\hline Fit Indices & Modell 1 & Modell 2 & Modell 3 \\
\hline Loglikelihood & -2716.02 & -2715.13 & -2714.76 \\
\hline $\mathrm{AIC}$ & 5448.04 & 5450.26 & 5457.52 \\
\hline$\Delta \chi^{2}$ & $\begin{array}{r}251.05^{* * *}(4 \\
\mathrm{df})\end{array}$ & $1.78(2 \mathrm{df})$ & $1.00(4 \mathrm{df})$ \\
\hline
\end{tabular}

Anmerkungen: $\mathrm{N}=1076$ (Schülerinnen und Schüler) 84 (Klassen) 47 (Schulen); unstandardisierte B Koeffizienten (SE); ${ }^{+} \mathrm{p} \leq .10,{ }^{*} \mathrm{p} \leq .05,{ }^{* *} \mathrm{p} \leq .01,{ }^{* * *} \mathrm{p} \leq .001$

\section{Diskussion}

Dieser Beitrag untersucht, ob die Entwicklung von internalisierendem und prosozialem Verhalten durch die Nutzung von Tagesschulangeboten beeinflusst wird und welche Rolle dabei die Interaktionen durch das Betreuungspersonal und die Verhaltensweisen der Klassenkameraden im Tagesschulangebot spielen. Während sich die dauerhafte Angebotsnutzung übereinstimmend mit Hypothese 1a auf die Entwicklung des internalisierenden Verhaltens auswirkte, zeigte sich für das prosoziale Verhalten kein Effekt, wodurch Hypothese 2a nicht bestätigt werden konnte. Der Befund, dass eine dauerhafte Nutzung des Tagesschulangebots einen unvorteilhaften Einfluss auf die Entwicklung von internalisierendem 
Verhalten ausübt, widerspricht den bisherigen Studien zu Tages- oder Ganztagschulen, die keinen Effekt der Angebotsnutzung auf die Entwicklung von sozioemotionalen Verhaltensstärken (Schüpbach et al., 2014) oder des Wohlbefindens (Fischer et al., 2011) fanden. Das Ergebnis steht auch im Widerspruch zur Metaanalyse von Durlak et al. (2010) zu after-school Programmen, die einen vorteilhaften Einfluss dieser Programme auf die Entwicklung der Selbstwahrnehmung und der Verbundenheit zur Schule ausmachte. Dass kein Effekt auf die Entwicklung des prosozialen Verhaltens vorliegt, deckt sich zwar mit den Befunden aus dem deutschsprachigen Raum (Fischer et al., 2011; Schüpbach et al., 2014), widerspricht aber dem positiven Effekt bei (Durlak et al., 2010). Da sich die bisherigen Arbeiten sowohl in der jeweiligen Stichprobe, dem Untersuchungszeitraum, den Messinstrumenten und dem analytischen Vorgehen teils stark unterscheiden, sind diese Unterschiede an dieser Stelle kaum zu erklären und erfordern weitere Untersuchungen, insbesondere zu möglichen Moderatoren.

Der nachteilige Effekt der dauerhaften Angebotsnutzung auf die Entwicklung von internalisierendem Verhalten wird zumindest teilweise durch das internalisierende Verhalten der Gleichaltrigen erklärt, wodurch Hypothese $1 \mathrm{~b}$ ebenfalls bestätigt wird: Während ein niedriges Niveau an internalisierendem Verhalten im Tagesschulangebot insgesamt zu einer Abnahme des internalisierenden Verhaltens der einzelnen Schülerinnen und Schüler führt und kaum Unterschiede zwischen den Gruppen feststellbar sind, zeigt bei einem erhöhten Niveau insbesondere die Gruppe der dauerhaften Angebotsnutzerinnen und -nutzer eine $\mathrm{Zu}$ nahme an internalisierenden Verhaltensweisen. Damit wurde ein schädlicher Einfluss durch die Gleichaltrigen (auch «peer contagion» genannt, vgl. Dishion \& Tipsord, 2011) nicht nur für externalisierendes Verhalten (Frei et al., 2016), sondern auch für internalisierendes Verhalten festgestellt. Hinsichtlich prosozialem Verhalten zeigte sich hingegen weder ein direkter Effekt durch das Niveau an prosozialem Verhalten der Gleichaltrigen im Angebot, noch zeigte sich eine Interaktion mit der Angebotsnutzung, wodurch Hypothese $2 \mathrm{~b}$ nicht bestätigt wurde. Möglicherweise sticht prosoziales Verhalten - zumindest nach der Operationalisierung in unserer Studie - weniger heraus als externalisierendes oder auch internalisierendes Verhalten, da die allermeisten Schülerinnen und Schüler die erfragten prosozialen Verhaltensweisen zeigen, während externalisierendes oder internalisierendes Verhalten vergleichsweise selten gezeigt wird. Zudem dürften problematische Verhaltensweisen mehr Störungen mit sich bringen und dadurch mehr Aufmerksamkeit generieren. 
Die Interaktionsqualität im Tagesschulangebot zeigte weder einen Effekt auf die Entwicklung des internalisierenden oder prosozialen Verhaltens, noch wurden die Auswirkungen einer dauerhaften Angebotsnutzung dadurch moderiert. Entsprechend konnten die Hypothesen 1c und 2c nicht bestätigt werden. Diese Ergebnisse stehen im Gegensatz zum Befund, dass die Wirkung der Angebotsnutzung auf die Entwicklung von externalisierendem Verhalten durch die Interaktionsqualität beeinflusst wird (Frei et al., 2016). Dieser Unterschied könnte darauf zurückgehen, dass insbesondere externalisierendes Verhalten eine unmittelbare Reaktion durch das Betreuungspersonal einfordert und als Folge in besonderem Masse durch die Interaktionen zwischen Betreuungspersonal und Schülerschaft beeinflusst wird. Die vorliegende Studie unterliegt einigen Einschränkungen:

(1) Der Untersuchungszeitraum war auf eine Dauer von einem Jahr begrenzt, weshalb längerfristige oder verzögerte Effekte nicht untersucht werden konnten.

(2) Die Teilnahme am Tageschulangebot wurde ausschliesslich über die generelle Angebotsnutzung operationalisiert. Die Breite der besuchten Angebote sowie das persönliche Engagement der Schülerinnen und Schüler blieben unberücksichtigt, ebenso wie die Intensität der Angebotsnutzung.

(3) Idealerweise würde das Verhalten der Gleichaltrigen als latent geschätzte Variable auf Klassenebene berücksichtigt, da die Aggregierung von Variablen einer niedrigeren Ebene die Standardfehler verzerren kann. Allerdings ist es mit MPlus derzeit nicht möglich, bei drei-Ebenen-Modellen einen Prädiktor auf mehreren Ebenen gleichzeitig zu spezifizieren.

(4) Die Einschätzung zur Interaktionsqualität basiert auf einer halbtägigen Beobachtung durch eine Person. Oh, Osgood und Smith (2015) empfehlen allerdings Beobachtungen zu mehreren Zeitpunkten und durch mehrere Beobachter, um die Reliabilität der Einschätzung zu erhöhen. Entsprechend könnte in unserer Studie die Bedeutung der Interaktionsqualität aufgrund von Messfehlern unterschätzt werden.

Erstmals im deutschsprachigen Raum wurde der Einfluss einer Nutzung des Angebots an Tages- oder Ganztagsschulen auf die Entwicklung von internalisierendem oder prosozialem Verhalten sowie möglicher Moderatoren anhand von Mehrebenenmodellen untersucht. Dabei zeigte eine dauerhafte Nutzung des Angebots eine unvorteilhafte Wirkung auf die Entwicklung des internalisieren- 
den Verhaltens im Falle eines erhöhten Niveaus an internalisierendem Verhalten bei den Gleichaltrigen. Dieser Befund zeigt auf, dass eine gute Durchmischung von Schülerinnen und Schülern mit und ohne Verhaltensauffälligkeiten angestrebt werden sollte. Zudem könnten Massnahmen geprüft werden, um solchen unvorteilhaften Effekten entgegen zu wirken (z.B. eine Erhöhung des Betreuungsschlüssels, eine Sensibilisierung des Personals oder spezifische Interventionen im Angebot).

\section{Literatur}

Byrne, B. M. (2012). Structural equation modeling with Mplus. Basic concepts, applications, and programming (Multivariate applications series). New York, NY: Routledge Taylor \& Francis Group.

Dishion, T. J. \& Tipsord, J. M. (2011). Peer Contagion in Child and Adolescent Social and Emotional Development. Annual Review of Psychology, 62 (1), 189-214.

Durlak, J. A., Weissberg, R. P. \& Pachan, M. (2010). A Meta-Analysis of After-School Programs That Seek to Promote Personal and Social Skills in Children and Adolescents. American journal of community psychology, 45 (3-4), 294-309.

EDK. (2013). Kantonsumfrage 2012/2013. Zugriff am 07.05.2017, von http://edudoc.ch/record/ 115194/files/2013.pdf

Fergusson, D. M., Horwood, L. J. \& Ridder, E. M. (2007). Conduct and attentional problems in childhood and adolescence and later substance use, abuse and dependence. Results of a 25-year longitudinal study. Drug and Alcohol Dependence, 88, S14-S26.

Fischer, N., Brümmer, F. \& Kuhn, H. P. (2011). Entwicklung von Wohlbefinden und motivationalen Orientierungen in der Ganztagsschule. Zusammenhänge mit der Prozess- und Beziehungsqualität in den Angeboten. In N. Fischer, H. G. Holtappels, E. Klieme, T. Rauschenbach, L. Stecher \& I. Züchner (Hrsg.), Ganztagsschule: Entwicklung, Qualität, Wirkungen. Längsschnittliche Befunde der Studie zur Entwicklung von Ganztagsschulen (StEG) (S. 227-245). Weinheim \& Basel: Beltz Juventa.

Fischer, N., Kuhn, H. P. \& Züchner, I. (2011). Entwicklung von Sozialverhalten in der Ganztagsschule. Wirkungen der Ganztagsteilnahme und der Angebotsqualität. In N. Fischer, H. G. Holtappels, E. Klieme, T. Rauschenbach, L. Stecher \& I. Züchner (Hrsg.), Ganztagsschule: Entwicklung, Qualität, Wirkungen. Längsschnittliche Befunde der Studie zur Entwicklung von Ganztagsschulen (StEG) (S. 246-266). Weinheim \& Basel: Beltz Juventa.

Frei, L., Schüpbach, M., Nieuwenboom, W. \& von Allmen, B. (2016). Extended Education and Externalizing Behavior. Utilization Intensity, Interaction Quality and Peers as Possible Moderators. IJREE - International Journal for Research on Extended Education, 4 (2), 92-109.

Frei, L., Schüpbach, M., von Allmen, B. \& Nieuwenboom, W. (2017). Außerunterrichtliche Bildungs- und Betreuungsangebote als Mittel gegen externalisierendes Verhalten? Die Bedeutung der Gleichaltrigen und des Betreuungspersonals an Deutschschweizer Tagesschulen. Manuskript zur Publikation eingereicht.

Fröhlich-Gildhoff, K. (2013). Verhaltensauffälligkeiten bei Kindern und Jugendlichen (2. Aufl.). Stuttgart: W. Kohlhammer.

Goodman, A., Lamping, D. L. \& Ploubidis, G. B. (2010). When to use broader internalising and externalising subscales instead of the hypothesised five subscales on the Strengths and Difficul- 
ties Questionnaire (SDQ): data from British parents, teachers and children. Journal of abnormal child psychology, 38 (8), 1179-1191.

Goodman, R. (1997). The Strengths and Difficulties Questionnaire. A Research Note. Journal of Child Psychology and Psychiatry, 38 (5), 581-586.

Goodman, R. (2001). Psychometric Properties of the Strengths and Difficulties Questionnaire. Journal of the American Academy of Child \& Adolescent Psychiatry, 40 (11), 1337-1345.

Harms, T., Jacobs, E. V. \& White, D. R. (1996). School-age care environment rating scale. New York: Teachers College Press.

Kanevski, R. \& von Salisch, M. (2011). Fördert die Ganztagsschule die Entwicklung sozialer und emotionaler Kompetenzen bei Jugendlichen? Zeitschrift für Erziehungswissenschaft, 14 (3), 237-259.

Kanning, U. P. (2002). Soziale Kompetenz - Definition, Strukturen und Prozesse. Zeitschrift für Psychologie, 210 (4), 154-163.

Kremer, K. P., Maynard, B. R., Polanin, J. R., Vaughn, M. G. \& Sarteschi, C. M. (2015). Effects of After-School Programs with At-Risk Youth on Attendance and Externalizing Behaviors. A Systematic Review and Meta-Analysis. Journal of Youth and Adolescence, 44 (3), 616-636.

Leadbeater, B. J., Thompson, K. \& Sukhawathanakul, P. (2016). Enhancing Social Responsibility and Prosocial Leadership to Prevent Aggression, Peer Victimization, and Emotional Problems in Elementary School Children. American journal of community psychology, 58 (3-4), 365-376.

McLeod, J. D. \& Kaiser, K. (2004). Childhood Emotional and Behavioral Problems and Educational Attainment. American Sociological Review, 69 (5), 636-658.

Metsäpelto, R.-L., Pakarinen, E., Kiuru, N., Poikkeus, A.-M., Lerkkanen, M.-K. \& Nurmi, J.-E. (2015). Developmental dynamics between children's externalizing problems, task-avoidant behavior, and academic performance in early school years. A 4-year follow-up. Journal of Educational Psychology, 107 (1), 246-257.

Muthén, L. K. \& Muthén, B. O. (1998-2012). Mplus User's Guide. Seventh Edition. Los Angeles, CA: Muthén \& Muthén.

Nelson, S. K., Layous, K., Cole, S. W. \& Lyubomirsky, S. (2016). Do unto others or treat yourself? The effects of prosocial and self-focused behavior on psychological flourishing. Emotion (Washington, D.C.), 16 (6), 850-861.

Oh, Y., Osgood, D. W. \& Smith, E. P. (2015). Measuring afterschool program quality using settinglevel observational approaches. The Journal of early adolescence, 35 (5-6), 681-713.

Reijntjes, A., Kamphuis, J. H., Prinzie, P. \& Telch, M. J. (2010). Peer victimization and internalizing problems in children: a meta-analysis of longitudinal studies. Child abuse \& neglect, 34 (4), 244-252.

Schüpbach, M. (2010). Ganztägige Bildung und Betreuung im Primarschulalter. Qualität und Wirksamkeit verschiedener Schulformen im Vergleich. Wiesbaden: VS Verlag für Sozialwissenschaften.

Schüpbach, M., Ignaczewska, J. \& Herzog, W. (2014). Sozio-emotionale Entwicklung von Ganztagsschulkindern auf der Primarschulstufe. Zeitschrift für Entwicklungspsychologie und Pädagogische Psychologie, 46 (1), 11-23.

Slopen, N., Kubzansky, L. D. \& Koenen, K. C. (2013). Internalizing and externalizing behaviors predict elevated inflammatory markers in childhood. Psychoneuroendocrinology, 38 (12), 2854-2862.

Tietze, W., Roßbach, H.-G., Stendel, M. \& Wellner, B. (2007). Hort- und Ganztagsangebote-Skala (HUGS). Feststellung und Unterstützung pädagogischer Qualität in Horten und außerunterrichtlichen Angeboten. Berlin: Cornelsen Scriptor.

Van den Noortgate, W., Opdenakker, M.-C. \& Onghena, P. (2005). The Effects of Ignoring a Level in Multilevel Analysis. School Effectiveness and School Improvement, 16 (3), 281-303. 
Weiß, R. H. \& Osterland, J. (2013). CFT 1-R. Grundintelligenztest Skala 1. Göttingen [u.a.]: Hogrefe.

\section{Angaben zu den Autorinnen und Autoren}

Lukas Frei, M. Sc., wissenschaftlicher Mitarbeiter am Institut für Psychologie der Universität Bern und Doktorand an der Universität Bamberg. Arbeitsschwerpunkte: Tagesschulen und sozio-emotionale Entwicklung. Analysen mit längsschnittlichen und geclusterten Daten

Marianne Schüpbach, Prof. Dr., Universität Bamberg, Lehrstuhlinhaberin für Grundschulpädagogik und -didaktik. Arbeitsschwerpunkte: Schulleistungsentwicklung sowie sozioemotionale Entwicklung der Schülerinnen und Schüler (Tagesschule/Ganztagsschule, Klassenwiederholung), pädagogische Qualität von Tagesschule/Ganztagsschule (Unterricht und Angebot) und Unterricht, Kooperation in Unterricht und Tagesschule/Ganztagsschule (u.a. Teamteaching, im inklusiven Unterrichtssetting)

Wim Nieuwenboom, Dr., wissenschaftlicher Mitarbeiter an der Fachhochschule Nordwestschweiz, Hochschule für Soziale Arbeit und an der Universität Bamberg, Lehrstuhl für Grundschulpädagogik und -didaktik. Arbeitsschwerpunkte: Psychische Gesundheit und Integration bei Kindern und Jugendlichen, Sucht und Resilienz, Quantitative Methoden, Leseforschung

Benjamin von Allmen, M Sc., wissenschaftlicher Mitarbeiter am Institut für Psychologie der Universität Bern und Doktorand an der Universität Bamberg. Arbeitsschwerpunkte: Schulleistungsentwicklung von Schülerinnen und Schülern an Tagesschulen, mündliches Sprachniveau und Schulleistung, Qualität an Tagesschulen, quantitative Analyseverfahren 


\section{Beitrag des Autors zu den Manuskripten}

Sämtliche Manuskripte entstanden im Rahmen des vom Schweizerischen Nationalfonds finanzierten Forschungsprojekts "EduCare-TaSe - Tagesschulen und Schulerfolg?" (SNF Nr. 100013-143797/1; Laufzeit: 1.3.2013-31.05.2017). Das Projekt stand unter der Leitung von Prof. Dr. Marianne Schüpbach und wurde durch zwei wissenschaftliche Mitarbeitende ergänzt: Anfänglich waren dies Benjamin von Allmen und Michelle Jutzi - letztere startete ab Sommer 2014 ein eigenes Forschungsprojekt und wurde durch Lukas Frei ersetzt. Zudem stand Dr. Wim Nieuwenboom als methodischer und statistischer Berater zur Verfügung. Die Erhebung und Aufbereitung der Daten (Befragung der Schul- und Angebotsleitungen, der Lehrpersonen, der Eltern, der Schülerinnen und Schüler; Erhebung der kognitiven Grundfähigkeiten) erfolgte durch das Forschungsteam und wurde größtenteils durch Benjamin von Allmen und Lukas Frei koordiniert. Einzig die Beobachtung im Tagesschulangebot wurde durch Benjamin von Allmen und Michelle Jutzi koordiniert. Sämtliche Manuskripte wurden von Lukas Frei verfasst und überarbeitet, wobei Diskussionen und Rückmeldungen innerhalb des Forschungsteams sowie Rückmeldungen von Seite der Zeitschriften berücksichtigt wurden.

Manuskript 1: Frei, L., Schüpbach, M., von Allmen, B. \& Nieuwenboom, W. (2016). Bildungsbezogene Erwartungen an Tagesschulen. Förderangebote an offenen Tagesschulen in der Deutschschweiz. Schweizerische Zeitschrift für Bildungswissenschaften, 38 (3), 549-567.

Von Marianne Schüpbach stammt die Idee, einen Artikel zur Schulentwicklung und zum Ausbau des Tagesschulangebots zu machen. Basierend auf dieser Idee entwickelte Lukas Frei unter Rücksprache mit dem Forschungsteam - die vorliegenden Fragestellungen. Die Analysemethoden wurden von Lukas Frei ausgewählt, wiederum unter Rücksprache mit dem Forschungsteam. Sämtliche Analysen wurden von Lukas Frei durchgeführt, mit punktueller Unterstützung durch Wim Nieuwenboom. 
Manuskript 2: Frei, L., Schüpbach, M., Nieuwenboom, W. \& von Allmen, B. (2016). Extended Education and Externalizing Behavior. Utilization Intensity, Interaction Quality and Peers as Possible Moderators. International Journal for Research on Extended Education, 4 (2), 92109.

Die Idee, den Einfluss der Nutzungsintensität im Tagesschulangebot auf die Entwicklung von sozialen Kompetenzen zu untersuchen, wurde bereits im Projektantrag durch Marianne Schüpbach festgehalten. Der Fokus auf externalisierendes Verhalten und auf die untersuchten Moderatoren wurde durch Lukas Frei vorgenommen. Die Idee für die Mehrebenenanalysen mit Cross-Level-Interaktionen stammt aus einem Workshop zu Mplus an der Universität Bern (durchgeführt von Dr. Jeannette Brodbeck) und wurde von Lukas Frei aufgenommen und an ein Modell mit drei Ebenen adaptiert. Die Analysen erfolgten durch Lukas Frei, mit punktueller Unterstützung durch Wim Nieuwenboom.

Manuskript 3: Frei, L., Schüpbach, M., von Allmen, B. \& Nieuwenboom, W. (2021). Außerunterrichtliche Bildungs- und Betreuungsangebote als Mittel gegen externalisierendes Verhalten? Die Bedeutung der Gleichaltrigen und des Betreuungspersonals an Deutschschweizer Tagesschulen. Psychologie in Erziehung und Unterricht, 68 (2), 117-134.

Manuskript 4: Frei, L., Schüpbach, M., Nieuwenboom, W. \& Allmen, B. von (2018). Sozioemotionale Entwicklung. In M. Schüpbach, L. Frei \& W. Nieuwenboom (Hrsg.), Tagesschulen (S. 213-228). Wiesbaden: Springer Fachmedien Wiesbaden

Auch der Vergleich der Entwicklung von sozialen Kompetenzen bei Schülerinnen und Schülern mit oder ohne Nutzung des Tagesschulangebots wurde bereits im Projektantrag festgehalten. Die theoretisch begründete Differenzierung zwischen externalisierendem, internalisierendem und prosozialem Verhalten wurde von Lukas Frei vorgenommen. Nachdem das zweite Manuskript die Interaktionen mit dem Betreuungspersonal und das Verhalten der Gleichaltrigen als 
Moderatoren identifizierte, wurden diese von Lukas Frei auch im dritten und vierten Manuskript aufgegriffen - zuerst im Hinblick auf externalisierendes, dann auf internalisierendes und prosoziales Verhalten. Da sich die Mehrebenenanalysen im zweiten Manuskript bewährt hatten, wurden sie von Lukas Frei auch in den Manuskripten 3 und 4 eingesetzt. Wim Nieuwenboom stand erneut beratend zur Seite. 